\title{
Effect of ultrasonic probe movement on tissue displacement measurements for muscle monitoring
}

\author{
By \\ Andy Huang, B.Sc., M.Eng. \\ A thesis submitted to the Faculty of Graduate and Postdoctoral Affairs in partial \\ fulfillment of the requirements for the degree of \\ Masters of Applied Science in Biomedical Engineering \\ Ottawa-Carleton Institute for Biomedical Engineering \\ Department of Systems and Computer Engineering \\ Carleton University \\ Ottawa, Ontario, Canada \\ December 2012
}

Copyright $\mathbb{C}$ Andy Huang, 2012 
Library and Archives

Canada

Published Heritage

Branch

395 Wellington Street

Ottawa ON K1A ON4

Canada
Bibliothèque et

Archives Canada

Direction du

Patrimoine de l'édition

395 , rue Wellington

Ottawa ON K1A ON4

Canada
Your file Votre référence

ISBN: 978-0-494-94247-5

Our file Notre référence

ISBN: $978-0-494-94247-5$
NOTICE:

The author has granted a nonexclusive license allowing Library and Archives Canada to reproduce, publish, archive, preserve, conserve, communicate to the public by telecommunication or on the Internet, loan, distrbute and sell theses worldwide, for commercial or noncommercial purposes, in microform, paper, electronic and/or any other formats.

The author retains copyright ownership and moral rights in this thesis. Neither the thesis nor substantial extracts from it may be printed or otherwise reproduced without the author's permission.
AVIS:

L'auteur a accordé une licence non exclusive permettant à la Bibliothèque et Archives Canada de reproduire, publier, archiver, sauvegarder, conserver, transmettre au public par télécommunication ou par l'Internet, prêter, distribuer et vendre des thèses partout dans le monde, à des fins commerciales ou autres, sur support microforme, papier, électronique et/ou autres formats.

L'auteur conserve la propriété du droit d'auteur et des droits moraux qui protege cette thèse. $\mathrm{Ni}$ la thèse ni des extraits substantiels de celle-ci ne doivent être imprimés ou autrement reproduits sans son autorisation.
In compliance with the Canadian Privacy Act some supporting forms may have been removed from this thesis.

While these forms may be included in the document page count, their removal does not represent any loss of content from the thesis.
Conformément à la loi canadienne sur la protection de la vie privée, quelques formulaires secondaires ont été enlevés de cette thèse.

Bien que ces formulaires aient inclus dans la pagination, il n'y aura aucun contenu manquant. 
The undersigned recommend to the

Faculty of Graduate Studies and Research acceptance of the thesis

\title{
Effect of ultrasonic probe movement on tissue displacement measurements for muscle monitoring
}

\author{
Submitted by Andy Huang \\ in partial fulfillment of the requirements for \\ the degree of Master of Applied Science in Biomedical Engineering
}

Thesis Supervisor

Dr. Yuu Ono

Chair, Department of Systems and Computer Engineering

Dr. Howard Schwartz

2012, Carleton University 


\begin{abstract}
This thesis studied the effect of lateral motion of ultrasonic probe on vertical displacement estimation of muscle tissue by means of phase sensitive method using ultrasound. The vertical displacement estimation can be used to monitor muscle activities during muscular contraction. A numerical simulation software was developed to simulate an ultrasound radio frequency signal based on summation of speckle echoes from ultrasound scatterers randomly distributed in a soft tissue. The numerical simulation, phantom simulation experiments and in vivo experiments were conducted to investigate errors caused by lateral probe motion and random noise in M-mode measurements. In addition, a moving average filter was tested in order to reduce the errors. It was shown that lateral motion distance less than $10 \mu \mathrm{m}$ per $\mathrm{M}$-mode frame resulted in constant error under the numerical simulation and experimental conditions used in this thesis. For the experimental conditions used in the thesis, the moving average filter reduced errors at all relevant signal-to-noise ratio (SNR) values for lateral motion distances of $1 \mu \mathrm{m} /$ frame and below. For lateral motion distances of $10 \mu \mathrm{m} /$ frame and above the moving average filter reduced the errors at lower SNR regions. However, it did not reduce the errors effectively at larger SNR regions since the lateral motion was the dominant error source in such case.
\end{abstract}




\section{Acknowledgements}

I would like to express my thanks to my supervisor Dr. Yuu Ono, Associate Professor of Systems and Computer Engineering, Carleton University for his expertise, guidance, encouragement, and support. I appreciate the time and effort which he provided over the course of my graduate studies.

Thank you to Dr. Adrian Chan and Graham Fraser for their technical assistance in the electromyography (EMG) experiments, and to Dr. Andy Adler for the use of the ultrasound imaging system.

I would also like to thank my colleague Zhen Qu for his help and technical assistance throughout my research. He was always willing to share his previous experience and offer insight into any encountered difficulties.

I would also like to extend my thanks and appreciation to Carleton University, and Natural Sciences and Engineering Research Council of Canada (NSERC) for the opportunity and financial support provided for the research.

I'd also like to give special thanks for my friends and family for their support, patience, and encouragement. 


\section{Table of Contents}

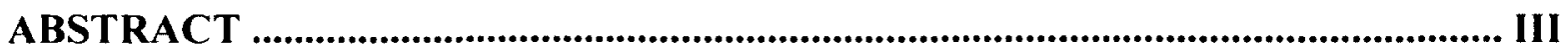

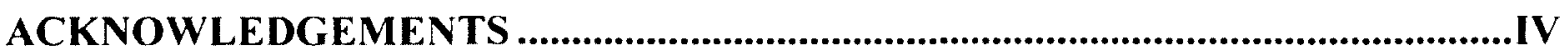

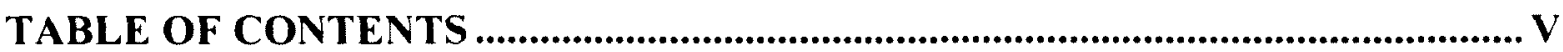

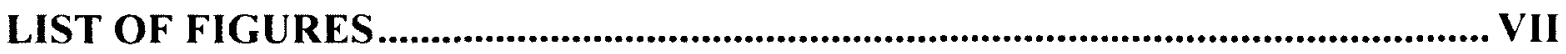

LIST OF ABBREVIATIONS ........................................................................................

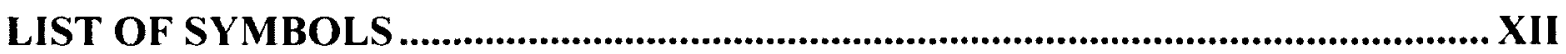

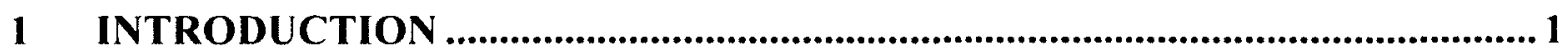

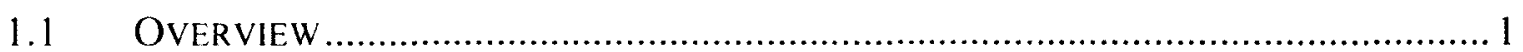

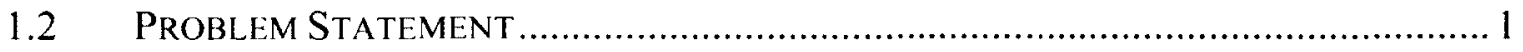

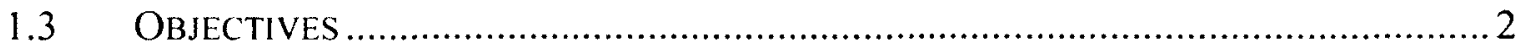

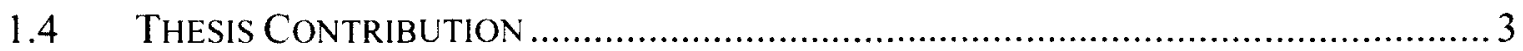

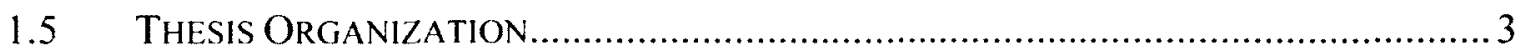

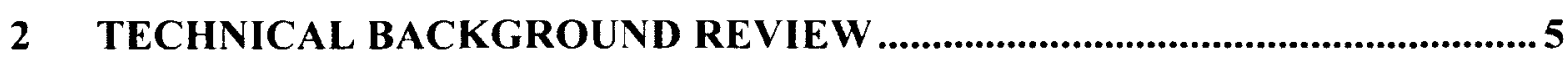

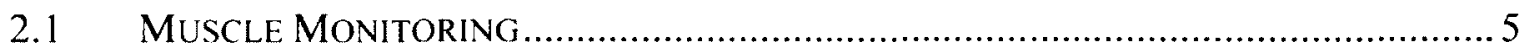

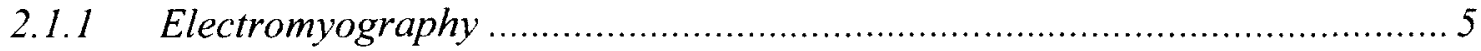

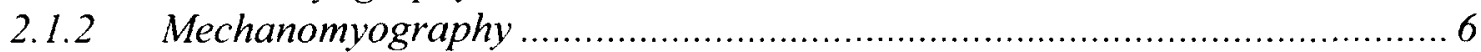

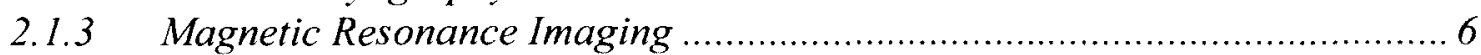

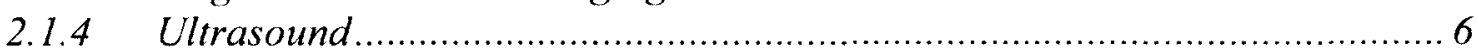

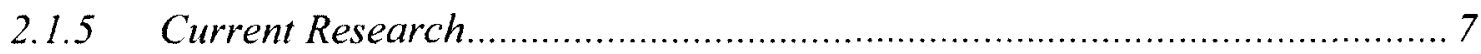

2.1.6 Uncertainty in Displacement Estimation from Ultrasound Imaging............ 8

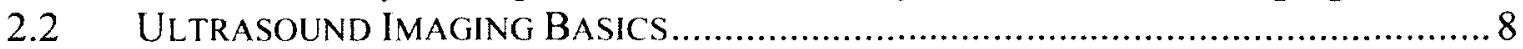

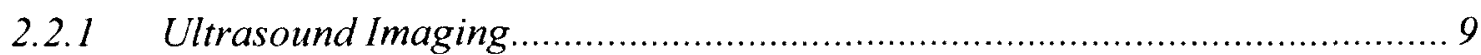

2.2.2 Tissue Vertical Displacement Estimation ............................................ 13

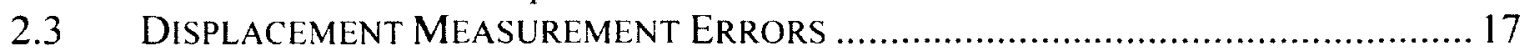

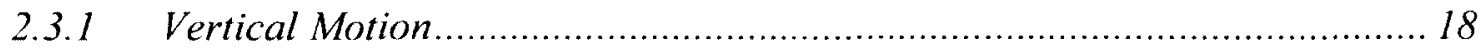

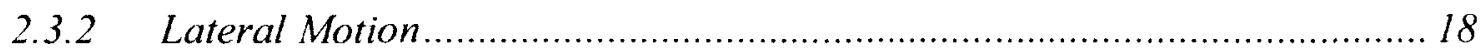

2.3.3 Rotation and Shear Motion..................................................................

3 FOCUSED ULTRASOUND BEAM WIDTH MEASUREMENT ......................20

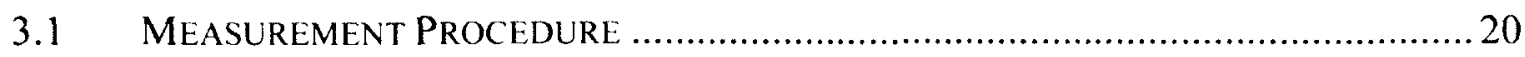

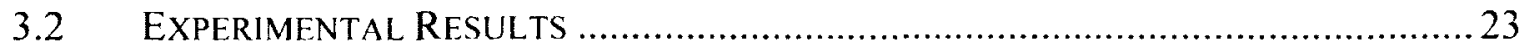

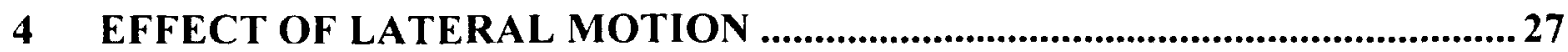

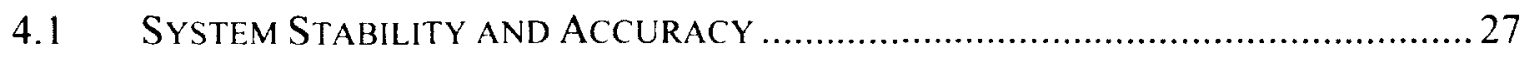

4.1.1 System Stability Experimental Procedure .............................................. 28

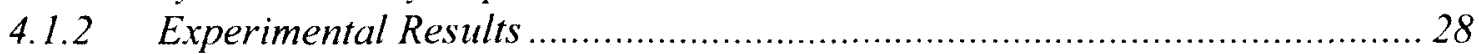

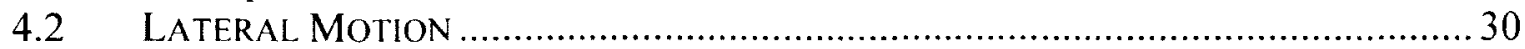

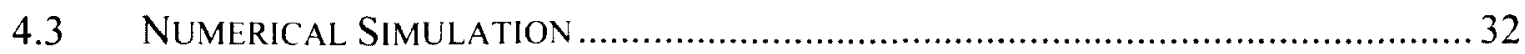




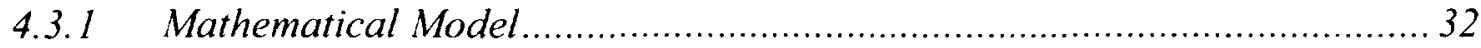

4.3.2 Phase Estimation Parameterization .................................................... 35

4.3.3 Noise Quantification............................................................... 36

4.3.4 Parameter setting for numerical simulation..................................... 37

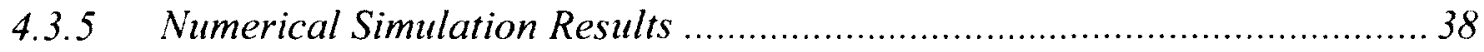

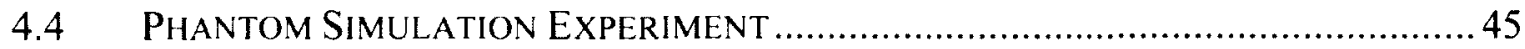

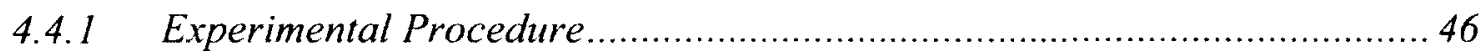

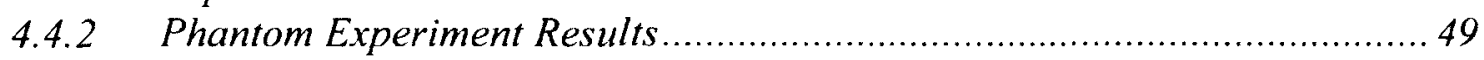

4.5 MOVING AVERAGE FILTER FOR RANDOM NOISE REDUCTION ........................51

$5 \quad V E R T I C A L$ DISPLACEMENT MEASUREMENTS.....................................55

5.1 PhANTOM SiMULATION EXPERIMENTS …................................................. 55

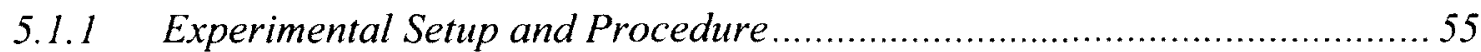

$5.1 .2 \quad$ Experimental Results .................................................................. 56

5.1.3 Moving Average Filter for Random Noise Error Reduction .....................66

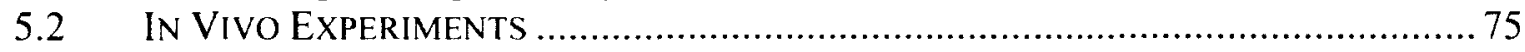

5.2.1 Experimental Setup and Procedure .................................................... 75

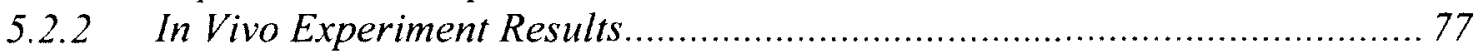

5.2.3 Moving Average Filter for Random Noise Error Reduction ......................84

6 CONCLUSION AND FUTURE WORK...................................................91

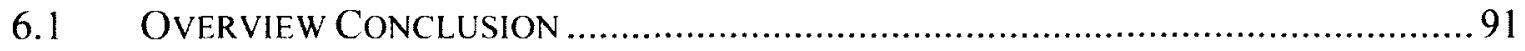

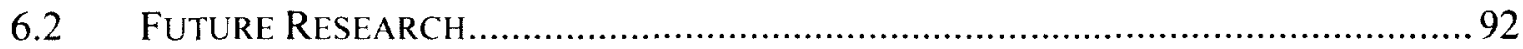

APPENDIX A: ULTRASOUND MUSCLE MONITORING .................................94

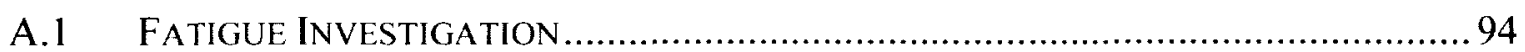

A.1.1 Measurement Setup and Experimental Procedure .................................. 95

A.1.2 Preliminary Results and Discussion................................................. 96

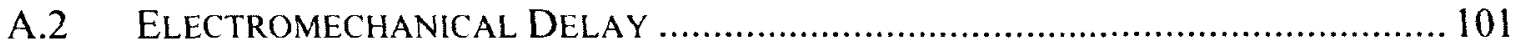

A.2.1 Measurement Setup and Experimental Procedure .............................. 102

A.2.2 Preliminary Results and Discussion.......................................... 102

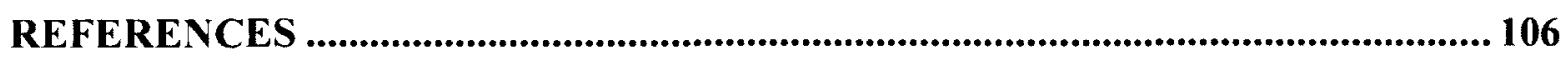




\section{List of Figures}

Figure 2.1 Ultrasound IMAGING MODEL SHOWS ULTRASOUND SCAN LINE, AS WELL AS

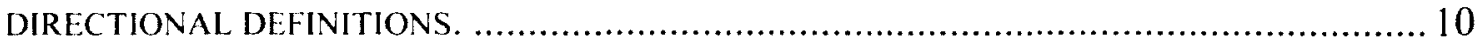

FIGURE 2.2 EXAMPLE OF ULTRASOUND RF SIGNAL AND A-MODE ENVELOPE ................. 10

FIGURE 2.3 EXAMPLE OF M-MODE IMAGE ........................................................... 12

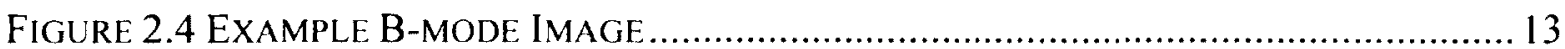

Figure 3.1 BEAM WIDTH IDEALIZED MEASUREMENT MODEL. THE Ultrasound PROBE IS MODELLED BY THE BLUE RECTANGULAR OBJECT, AND THE POINT REFLECTOR BY THE RED CIRCLE. THE DOTTED LINES MARK THE BEAM WIDTH OF THE TRANSMITTED ULTRASOUND BEAM.

FIGURE 3.2 EXPERIMENT SETUP FOR BEAM WIDTH MEASUREMENT. IN THIS SETUP, THE RAZOR IS SITUATED TO MEASURE THE BEAM WIDTH IN THE MECHANICAL FOCUSING DIRECTION.

FIGURE 3.3 REFLECTION ECHO PROFILE FROM RAZOR IN ELECTRICAL FOCUSING DIRECTION

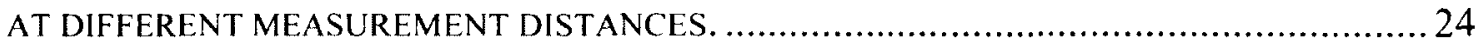

FIGURE 3.4 REFLECTION ECHO PROFILE FROM RAZOR IN MECHANICAL FOCUSING DIRECTION

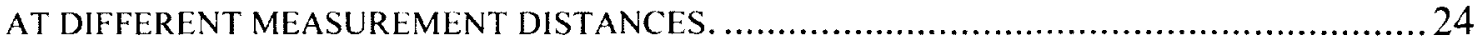

FIGURE 3.5 TRANSMITTED ULTRASOUND BEAM WIDTH IN ELECTRICAL AND MECHANICAL

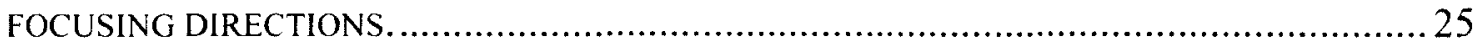

Figure 3.6 Razor Echo Amplitude Profile, Mechanical Focusing ...................... 26

FIgURE 4.1 EXPERIMENT StabILITY SETUP......................................................... 28

Figure 4.2 Experimental Setup Measurement Error, Phantom \# 1 Measurement29

Figure 4.3 Experimental Setup Measurement Error, Phantom \#2 Measurement 30

FIGURE 4.4 MEASUREMENT CONFIGURATION AND DIRECTION. THE PROBE LOCATION AND MEASUREMENT AREA BEFORE (A) AND AFTER (B) LATERAL MOTION.

FIGURE 4.5 TWO-DIMENSIONAL ULTRASOUND MODEL AND SCATTERER AXIS DEFINITION. THE $(0,0)$ COORDINATE CORRESPONDS TO A SCATTERER LOCATION IN THE TOP LEFT CORNER OF THE MEDIUM

FigURE 4.6 SimULATED AND MEASURED ULTRASOUND RF SiGNAL. THE SIMULATED SIGNAL (TOP) AND THE MEASURED SIGNAL (BOTTOM) ARE SHOWN ................................... 39

Figure 4.7 Simulated AND MEASURED ULTRASOUND RF SIGNAL SPECTRUM. THE SPECTRAL CONTENT OF THE SIMULATED ULTRASOUND RF SIGNAL IS SHOWN IN RED, WHILE THE SPECTRAL CONTENT OF THE MEASURED RF SIGNAL IS SHOWN IN DOTTED BLUE.

Figure 4.8 Simulation Results. Phase VS SNR data POINTS SHOWN IN RED. The SOLID LINE INDICATES THE VALUE OF 1.813 WHICH IS THE THEORETICAL MAXIMUM PHASE RMS DUE TO PHASE WRAPPING.

FIGURE 4.9 PHASE RMS VS LATERAL MOTION FOR DIFFERENT SNR LEVELS ................ 44

FIGURE 4.103 -W\% Agar PHANTOM WITH 3-W\% GRAPHITE POWDER .......................... 45

Figure 4.11 MOdEL OF PHANTOM EXPERIMENT SETUP. PHANTOM IS PLACED IN WATER BATH WHILE MECHANICAL STAGE MOVES PROBE LATERALLY AT A CONSTANT

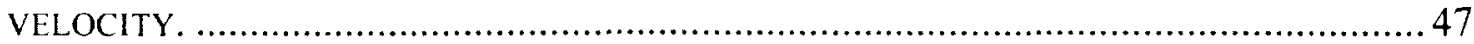

Figure 4.12 Phantom Motion NoISE Experimental Setup. The phantom is SUBMERGED IN A WATER BATH. THE PROBE IS SECURED TO A METAL HOLDER AND LOWERED UNTIL MEASUREMENT SURFACE IS SUBMERGED. 
Figure 4.13 PhaSe RMS AND SNR PLOT GENERATED FROM PHANTOM EXPERIMENT AND NUMERICAL SIMULATION. RESULTS FROM THE PHANTOM EXPERIMENT ARE IN SHOWN IN RED WHILE THE RESULTS FROM THE SIMULATION ARE SHOWN IN BLUE. THE HORIZONTAL BLACK LINE SHOWS THE THEORETICAL MAXIMUM SNR THRESHOLD..... 50

Figure 4.14 Phantom Experimental Results With and Without Moving AVERage FILTER OF 5 SAMPles. THE ORIGINAL DATA ARE IN BLUE, WHILE THE MOVING AVERAGE RESULTS ARE GIVEN IN RED....................................................... 53

FIGURE 5.1 ULTRASOUND RF SIGNAL FROM PHANTOM MEASUREMENT ........................ 58

FigurE 5.2 VERTICAL DISPLACEMENT OBSERVED IN PHANTOM BY PLEXIGLAS COMPRESSION. 58

FIGURE 5.3 DISTANCE OF PHANTOM SURFACE DISTANCE FROM ULTRASOUND PROBE DURING Plexiglas COMPRESSION. CROSS-CORRELATION ESTIMATE IS SHOWN IN SOLID BLUE LINE, AND QUADRATURE DEMODULATION IS SHOWN IN RED DASHED LINE. 60

FIGURE 5.4 PHANTOM VERTICAL DISPLACEMENT FROM HAND COMPRESSION USING A QUADRATURE DETECTION. 63

FIGURE 5.5 DISTANCE OF PHANTOM SURFACE DISTANCE FROM ULTRASOUND PROBE DURING HAND COMPRESSION. CROSS-CORRELATION ESTIMATE IS SHOWN IN SOLID BLUE LINE, AND QUADRATURE DEMODULATION IS SHOWN IN RED DASHED LINE.

Figure 5.6 DisPlacement of PHANTOM DUE TO Plexiglas COMPRESSION WITH MOVING AVERAGE FILTER.

FIGURE 5.7 DISTANCE OF PHANTOM SURFACE DISTANCE FROM ULTRASOUND PROBE DURING PleXiglas COMPRESSION WITH MOVING AVERAGE FILTER. CROSS-CORRELATION ESTIMATE IS SHOWN IN SOLID BLUE LINE, AND QUADRATURE DEMODULATION IS SHOWN IN RED DASHED LINE. 68

FIGURE 5.8 INSTANTANEOUS DISPLACEMENT ACROSS DEPTH BY PLEXIGLAS COMPRESSION WITHOUT MOVING AVERAGE FILTER. TOP PLOT SHOWS THE DISPLACEMENT ACROSS DEPTH WHEN THE PHANTOM IS STATIC. BOTTOM PLOT SHOWS THE DISPLACEMENT ACROSS DEPTH AS THE PHANTOM IS UNDERGOING COMPRESSION

FIGURE 5.9 INSTANTANEOUS DISPLACEMENT ACROSS DEPTH BY PLEXIGLAS COMPRESSION WITH MOVING AVERAGE FILTER. TOP PLOT SHOWS THE DISPLACEMENT ACROSS DEPTH WHEN THE PHANTOM IS STATIC. BOTTOM PLOT SHOWS THE DISPLACEMENT ACROSS DEPTH AS THE PHANTOM IS UNDERGOING COMPRESSION.

FIGURE 5.10 PHANTOM VERTICAL DISPLACEMENT FROM HAND COMPRESSION WITH MOVING AVERAGE FILTERING 72

FIGURE 5.11 DiSTANCE OF PHANTOM SURFACE FROM ULTRASOUND PROBE DURING HAND COMPRESSION WITH MOVING AVERAGE FILTERING. CROSS-CORRELATION ESTIMATE IS SHOWN IN SOLID BLUE LINE, AND QUADRATURE DEMODULATION IS SHOWN IN RED DASHED LINE. 72

FIGURE 5.12 INSTANTANEOUS DISPLACEMENT ACROSS DEPTH BY HAND COMPRESSION WITHOUT MOBBING AVERAGE FILTERING. TOP PLOT SHOWS THE DISPLACEMENT ACROSS DEPTH WHEN THE PHANTOM IS STATIC. BOTTOM PLOT SHOWS THE DISPLACEMENT ACROSS DEPTH AS THE PHANTOM IS UNDERGOING COMPRESSION. .....73

FIGURE 5.13 INSTANTANEOUS DISPLACEMENT ACROSS DEPTH FOR HAND COMPRESSION AFTER MOVING AVERAGE FILTERING. TOP PLOT SHOWS THE DISPLACEMENT ACROSS DEPTH WHEN THE PHANTOM IS STATIC. BOTTOM PLOT SHOWS THE DISPLACEMENT ACROSS DEPTH AS THE PHANTOM IS UNDERGOING COMPRESSION. ..........................74

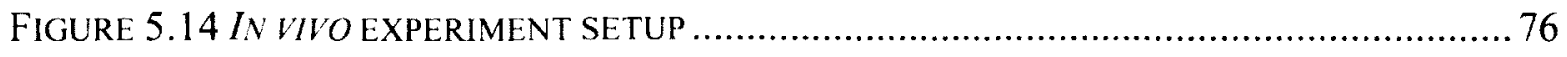


Figure 5.15 B-MODE IMAGE OF LEFT FOREARM. THE TARGET MUSCLE OF INTEREST AND BONE IS SHOWN IN RED. THE DASHED WHITE VERTICAL LINE INDICATES THE M-MODE MEASUREMENT VIEW.

FIGURE 5.16 MUSCLE VERTICAL DISPLACEMENT DURING DYNAMIC CONTRACTION.......... 78

FIGURE 5.17 MUSCLE VERTICAL DISPLACEMENT DURING DYNAMIC CONTRACTION AFTER BONE SUBTRACTION 80

FiguRE 5.18 ESTIMATED DISPLACEMENT AT DEPTH OF 15.0MM. TOP PLOT SHOWS THE ORIGINAL DATA WITHOUT BONE SUBTRACTION. BOTTOM PLOT SHOWS THE DATA AFTER BONE SUBTRACTION. CROSS-CORRELATION ESTIMATE IS SHOWN IN SOLID BLUE LINE, AND QUADRATURE DEMODULATION IS SHOWN IN RED DASHED LINE. .......81

FIGURE 5.19 MUSCLE VERTICAL DISPLACEMENT DURING ISOMETRIC CONTRACTION WITHOUT BONE SUBTRACTION.

FIGURE 5.20 MUSCLE VERTICAL DISPLACEMENT DURING ISOMETRIC CONTRACTION WITH BONE SUBTRACTION. 82

FIGURE 5.21 ESTIMATED DISPLACEMENT AT DEPTH OF 13.2MM DURING ISOMETRIC CONTRACTION. TOP PLOT SHOWS THE ORIGINAL DATA WITHOUT BONE SUBTRACTION. BOTTOM PLOT SHOWS THE DATA AFTER BONE SUBTRACTION. CROSS-CORRELATION ESTIMATE IS SHOWN IN SOLID BLUE LINE, AND QUADRATURE DEMODULATION IS SHOWN IN RED DASHED LINE. .83

FIGURE 5.22 ESTIMATED DISPLACEMENT FROM QUADRATURE DEMODULATION FROM DYNAMIC CONTRACTION EXPERIMENT AFTER BONE SUBTRACTION AND MOVING AVERAGE FILTER.

FigurE 5.23 DiSPLACEMENT AT MUSCLE-MUSCLE INTERFACE DURING DYNAMIC CONTRACTION AFTER BONE SUBTRACTION AND MOVING AVERAGE FILTER. CROSSCORRELATION ESTIMATE IS SHOWN IN SOLID BLUE LINE, AND QUADRATURE DEMODULATION IS SHOWN IN RED DASHED LINE...

85

FIGURE 5.24 INSTANTANEOUS DISPLACEMENT ESTIMATE FROM QUADRATURE DEMODULATION FOR ISOTONIC CONTRACTION EXPERIMENT. TOP PLOT IS AT A TIME INDEX WHERE THE MUSCLE WAS AT REST. BOTTOM PLOT IS WHEN THE MUSCLE IS IN THE PROCESS OF BEING CONTRACTED. .86

FIGURE 5.25 INSTANTANEOUS DISPLACEMENT ESTIMATE FROM QUADRATURE DEMODULATION FOR ISOTONIC CONTRACTION EXPERIMENT AFTER MOVING AVERAGE FILTER. TOP PLOT IS AT A TIME INDEX WHERE THE MUSCLE WAS AT REST. BOTTOM PLOT IS WHEN THE MUSCLE IS IN THE PROCESS OF BEING CONTRACTED. 87

FIGURE 5.26 ESTIMATED DISPLACEMENT FROM QUADRATURE DEMODULATION FROM ISOMETRIC CONTRACTION EXPERIMENT AFTER BONE SUBTRACTION AND MOVING AVERAGE FILTER. .88

FIGURE 5.27 DISPLACEMENT AT MUSCLE-MUSCLE INTERFACE DURING ISOMETRIC CONTRACTION WITH BONE SUBTRACTION AND MOVING AVERAGE FILTER. CROSSCORRELATION ESTIMATE IS SHOWN IN SOLID BLUE LINE, AND QUADRATURE DEMODULATION IS SHOWN IN RED DASHED LINE... 88

FIGURE 5.28 INSTANTANEOUS DISPLACEMENT ESTIMATE FROM QUADRATURE DETECTION FOR ISOMETRIC MOTION EXPERIMENT. TOP PLOT IS AT A TIME INDEX WHERE THE MUSCLE WAS AT REST. BOTTOM PLOT IS WHEN THE MUSCLE IS IN THE PROCESS OF BEING CONTRACTED. 89

FIGURE 5.29 INSTANTANEOUS DISPLACEMENT ESTIMATE FROM QUADRATURE DETECTION FOR ISOMETRIC MOTION EXPERIMENT AFTER MOVING AVERAGE FILTER. TOP PLOT IS 
AT A TIME INDEX WHERE THE MUSCLE WAS AT REST. BOTTOM PLOT IS WHEN THE MUSCLE IS IN THE PROCESS OF BEING CONTRACTED ......................................... 90 


\section{List of Abbreviations}

\begin{tabular}{|c|c|}
\hline Abbreviation & Definition \\
\hline $2-\mathrm{D}$ & Two-dimensional \\
\hline A-Mode & Amplitude Modality of ultrasound imaging \\
\hline $\mathrm{ADC}$ & Analog to Digital Conversion \\
\hline AWGN & Additive White Gaussian Noise \\
\hline B-Mode & Brightness Modality of ultrasound imaging \\
\hline EMG & Electromyography \\
\hline FWHM & Full Width Half Maximum \\
\hline M-Mode & Motion Modality of ultrasound imaging \\
\hline MMG & Mechanomyography \\
\hline MRI & Magnetic Resonance Imaging \\
\hline RF & Radio Frequency \\
\hline RMS & Root Mean Square \\
\hline sEMG & Surface Electromyography \\
\hline SNR & Signal to Noise Ratio \\
\hline $\mathrm{W} \%$ & Percent by weight \\
\hline
\end{tabular}




\section{List of Symbols}

\begin{tabular}{|c|c|c|}
\hline Abbreviation & Definition & Units \\
\hline$A$ & Received ultrasound signal amplitude & \\
\hline$\alpha_{k}$ & Amplitude factor of echo from scatterer $k$ & \\
\hline$\beta$ & Bandwidth factor of transmitted ultrasonic pulse & $\mathrm{Hz}^{2}$ \\
\hline$c$ & Ultrasound propagation velocity & $\mathrm{m} / \mathrm{s}$ \\
\hline$\Delta d$ & $\begin{array}{l}\text { Estimated vertical displacement between two } \\
\text { consecutive frames }\end{array}$ & $\mathrm{m}$ \\
\hline$f_{\text {dem }}$ & Frequency of reference signal & $\mathrm{Hz}$ \\
\hline$\overline{f_{c}}$ & $\begin{array}{c}\text { Estimated mean center frequency of transmitted } \\
\text { ultrasound signal }\end{array}$ & $\mathrm{Hz}$ \\
\hline I & In-phase demodulation ultrasound signal & \\
\hline$k$ & $\begin{array}{l}\text { Index number of scatterer in simulated measurement } \\
\qquad \text { object }\end{array}$ & \\
\hline$L P F$ & Low-pass filtering & \\
\hline$m$ & Frame index of ultrasound measurement & \\
\hline$n$ & Ultrasound RF signal sample index & \\
\hline$N$ & Total number of samples in arbitrary signal $x$ & \\
\hline$P$ & Transmitted ultrasonic pulse signal & \\
\hline$Q$ & Quadrature demodulation ultrasound signal & \\
\hline
\end{tabular}




\begin{tabular}{|c|c|c|}
\hline$\phi$ & $\begin{array}{l}\text { Phase shift between transmitted and received ultrasound } \\
\qquad \text { RF signal }\end{array}$ & radians \\
\hline$\phi_{k}$ & Phase shift due to scatterer $k$ & radians \\
\hline$r_{n, m}$ & $\begin{array}{l}\text { Complex autocorrelation at depth lag } n \text { and temporal lag } \\
\qquad m\end{array}$ & \\
\hline$S_{k}$ & Measured ultrasound RF signal from scatterer $k$ & \\
\hline$s_{r}$ & Received ultrasound RF signal & \\
\hline$s_{r e f}$ & Reference signal used in quadrature detection & \\
\hline$\sigma$ & Standard deviation of ultrasound along the temporal axis & radians \\
\hline$t$ & Time after pulse transmission & seconds \\
\hline$T_{s}$ & Ultrasound RF signal sample period & seconds \\
\hline$\tau_{k}$ & $\begin{array}{l}\text { Time delay from ultrasound pulse transmission to arrival } \\
\qquad \text { at scatterer } k\end{array}$ & seconds \\
\hline$u$ & Complex baseband signal from quadrature detection & \\
\hline$U$ & $\begin{array}{l}\text { Envelope of complex baseband signal from quadrature } \\
\text { detection }\end{array}$ & \\
\hline$W$ & Model of ultrasound beam profile in lateral direction & \\
\hline$\omega_{c}$ & Ultrasonic angular center frequency & radian \\
\hline$\omega_{\text {dem }}$ & Angular frequency of reference signal & radians \\
\hline$\Delta \omega$ & $\begin{array}{l}\text { Angular frequency difference between ultrasound and } \\
\text { reference signal }\end{array}$ & radians \\
\hline
\end{tabular}




\begin{tabular}{|c|c|c|}
\hline$X$ & Width of ultrasound beam & $\mathrm{m}$ \\
\hline$x_{c}$ & Center location of ultrasound probe along the $x$-axis & $\mathrm{m}$ \\
\hline$x_{k}$ & Location of scatterer $k$ along the $x$-axis & $\mathrm{m}$ \\
\hline$x_{n}$ & Sample $n$ of arbitrary signal $x$ & \\
\hline$z_{k}$ & Location of scatterer $k$ along the $z$-axis & $\mathrm{m}$ \\
\hline
\end{tabular}




\section{Introduction}

This chapter provides an overview as well as outlining the thesis organization. The objective and contributions will also be presented.

\subsection{Overview}

Muscle properties and behavior are used in a wide variety of different applications such as the diagnosis of neuromuscular disease, rehabilitation, therapy, and prosthesis control. The variation in the measured physical characteristics of the muscle can be indicative of changes in muscle health or activity. The motivation behind the research presented in this thesis is the ongoing need to improve accuracy and reliability of measurement of muscle characteristics. An important consideration in muscle measurement is the potential error sources present in the measurement setup. Identification and understanding of the errors introduced from different sources can assist in the design of an appropriate setup for the desired application.

\subsection{Problem Statement}

One parameter which represents muscle activity is the internal displacement (thickness variation) of a muscle. The change in thickness of the muscle is correlated with body movement [1]. Ultrasound Radio Frequency (RF) signals can be used to measure the vertical displacement, or displacement in a direction parallel to the ultrasound beam propagation. Lateral motion corresponds to movement of the measurement area in a direction perpendicular to ultrasound beam propagation and cannot be measured from the ultrasound RF signal. However, the lateral motion results in estimation of artificial motion unrelated to desired measured muscle activity. This lateral motion can result from both movement of the ultrasound probe as well as movement 
of the muscle fiber itself during muscle contraction. This erroneous detected movement due to lateral motion can result in false detection of muscle activity and inaccurate measurement of muscle thickness. The research in this thesis is aimed at acquiring a better understanding of the errors caused by lateral motion and improving the accuracy of muscle measurement characteristics using ultrasound.

\subsection{Objectives}

The primary goal of the thesis is to determine the effect of lateral motion on phase sensitive displacement estimation of muscle using ultrasound imaging. In addition, we also want to investigate methods to improve the accuracy of the measured results.

Towards this goal, numerical and phantom simulations are performed to examine the effect of the lateral motion in a controlled setting on a static object. A model-based computer simulation was designed to allow investigation of the effect of lateral motion in a strictly controlled environment. Phantom simulation experiments were then designed to investigate the effect in a more realistic scenario using a clinical ultrasound machine, and an error reduction method was investigated. Finally, in vivo experiments were performed to examine the effect of lateral movement on ultrasound measurement of muscle contraction at a forearm of a human subject. These results were also used to verify the effect of the moving average filter to reduce measurement error. 


\subsection{Thesis Contribution}

The following are the main contributions presented in the thesis:

- The effect of lateral probe motion on muscle displacement measurements was investigated. The investigation involved determining the impact of errors introduced by lateral probe motion.

- A software simulated environment was developed to simulate ultrasound RF signals. The development extended prior work on 1-D model to an 2-D environment for use in the investigation of errors caused by lateral probe motion

- An experimental procedure was developed for ultrasound measurement with nonstationary probe.

- A moving average filter was proposed for the reduction of random noise in ultrasound measurements without exacerbating errors caused by lateral motion.

\subsection{Thesis Organization}

Chapter 1 presents the overall concepts and topic of the thesis.

Chapter 2 provides general technical background about ultrasound imaging along with an overview of muscle monitoring. Previous research into ultrasound imaging errors related to probe motion is also discussed.

Chapter 3 presents the evaluation of the ultrasound measurement beam width for the clinical ultrasound machine used in this thesis.

Chapter 4 investigates the errors caused by lateral motion through numerical and phantom simulations on objects with no internal displacement. The moving average filter is also proposed to reduce random measurement noise without aggravating errors caused by lateral motion. 
Chapter 5 discusses the effect of lateral motion on measurement of objects under compression or contraction. Phantom simulation and in vivo experiments to measure the muscle displacement through ultrasound measurements are discussed. The effect of the moving average filter to reduce the random noise and improve measurement accuracy is also presented.

Chapter 6 concludes the research presented in this thesis. The major results are summarized, and improvements for future work are discussed. 


\section{Technical Background Review}

In this chapter, an introduction to muscle monitoring is presented along with previous research. Afterwards, the basic concept of ultrasound imaging is presented. Finally, an introduction of displacement estimation errors due to undesired motion of an ultrasonic probe used or a body under examination is provided.

\subsection{Muscle Monitoring}

Muscle monitoring uses different imaging modalities to capture a wide range of information about muscles. This information can be used for various applications, such as rehabilitation, therapy, diagnosis of neuromuscular diseases, and prosthetic control. In the following section, four common measurement methods used in muscle monitoring are introduced: electromyography (EMG), mechanomyography (MMG), magnetic resonance imaging (MRI), and ultrasound.

\subsubsection{Electromyography}

Electromyography $(\mathrm{EMG})$ is the measurement of the electrical activity generated during skeletal muscle contraction [2]. The measurement of the electrical activity is conducted by invasive or non-invasive electrodes [3,4]. Invasive electrodes, such as needle electrodes, pierce into the muscle tissue to obtain electrical measurements close to the muscle of interest. These electrodes generally have a small surface area and have a high spatial resolution. Non-invasive measurement uses surface electrodes to measure the electrical activity and is referred to as surface EMG (sEMG). These surface electrodes measure an average of the electrical activity of the muscle tissue under the surface area of the electrode and therefore have relatively poor 
spatial resolution compared to invasive needle electrodes. The electrical activity is also measured through the intervening muscle and fat layers which results in a tissue filtering effect on the electrical signal. While sEMG; electrodes has comparatively poor spatial resolution compared to needle electrodes and must deal with tissue filtering effects, sEMG is widely used due to its non-invasive nature.

\subsubsection{Mechanomyography}

Mechanomyography (MMG) is the measurement of the detectable mechanical surface motion that results from skeletal muscle contraction [5]. A wide variety of tools can be applied to measure the motion, such as optical distance sensors, accelerometers, piezoelectric contact sensors, and microphones [6]. MMG measurements therefore provide an external representation of the underlying muscle activity.

\subsubsection{Magnetic Resonance Imaging}

Magnetic resonance imaging (MRI) utilizes nuclear magnetic resonance to produce a non-invasive high resolution image of the internal muscle structure [7]. MRI can therefore provide information regarding the mechanical properties of muscles even within the body. MRI method has high operating cost and no portability.

\subsubsection{Ultrasound}

Ultrasound imaging uses high frequency acoustic waves to capture non-invasive images of the internal muscle structure. The basics of ultrasound imaging are described in Section 2.2. Motion tracking $[8,9]$ to measure muscle activity and strain measurement of muscle stiffness $[10,11]$ are two common usages of ultrasound imaging. Motion tracking from ultrasound 
imaging can be performed through Doppler imaging [12], correlation [8, 9], B-mode images analysis [1, 13-15], and peak or speckle tracking [16].

\subsubsection{Current Research}

Each of the above methods focuses on distinct aspects of the muscle; therefore they are suitable for different areas of research. EMG collects information about the electrical activity of the muscle and is a popular tool in fatigue measurement $[17,18]$. EMG has also been used in gait analysis to measure abnormalities in movement due to diseases $[19,20]$. MMG is used to measure the surface activity produced during muscular contraction. MMG is also used in the measurement of fatigue [21], along with detection and analysis of muscle activity [22, 23] as well as prosthesis control [24]. Research has also been performed on the utilization of EMG and MMG in conjunction with each other to combine the information about the electrical and mechanical activity of the muscles during contraction [25-28]. While MRI is not a popular research tool due to the above-mentioned issues, it can provide images of the interior muscle structure and has been used in research on disease diagnosis and monitoring $[29,30]$ and analysis of muscle behaviour during movement $[31,32]$.

Ultrasound imaging is used in motion tracking $[8,9]$, fatigue detection $[1,13]$ muscle strain estimation $[10,11]$. In addition, ultrasound imaging has been used in measurement of muscle tendon length $[16,33]$, three-dimensional imaging of muscle structure [34], and measurement of blood flow [35-37]. It has also been used in measurement of muscle elastic properties for disease diagnosis and fatigue detection [38, 39]. Ultrasound has also been combined with EMG to measure the electromechanical delay, or the time difference between electrical and mechanical activity of the muscle [40]. 


\subsubsection{Uncertainty in Displacement Estimation from Ultrasound Imaging}

Prior work in the area of uncertainty and accuracy in displacement estimation from ultrasound imaging has been focused on ultrasound B-mode images. The research has been focused on evaluation of accuracy of cross-correlation [41], study of factors that affect displacement accuracy [42], and the use of non-linear filtering to improve displacement estimation accuracy [43]. No previous work has been found on uncertainty estimation in displacement from ultrasound $\mathrm{M}$-mode images. The different ultrasound image modes are explained in further detail in the following section.

\subsection{Ultrasound Imaging Basics}

Ultrasound imaging utilizes the reflection of high frequency acoustic waves to create an image. These waves are generated by an ultrasonic transducer within an ultrasound probe. As the transmitted wave traverses the interface between two media it results in a partial reflection of the incident sound wave [44]. In addition to reflections at the media interfaces, scatterers within the medium can also result in formation of reflections as the acoustic wave travels across them. The size of the scatterers are often close to or smaller than the wavelength of the ultrasound wave and result in the formation of complex reflections known as scattering or speckle echoes $[45,46]$.

In the following section the application of these reflections for ultrasound image formation is discussed. Common ultrasound imaging modalities are introduced, along with the process of estimation of vertical displacement from the acquired ultrasound data. 


\subsubsection{Ultrasound Imaging}

Ultrasound imaging systems utilize the received reflection echoes to obtain non-invasive image of the internal structure of an object. From prior knowledge of acoustic wave propagation velocity and measurement of the time delay between transmission of the ultrasound wave and reception of the echoes, the distance from the ultrasound probe to reflection source can be determined [46]. The ultrasound image is generated based on this distance information alongside the amplitude of the measured reflection.

The transmitted ultrasound wave is beam shaped to enforce wave propagation in a particular direction. This shaping of the beam results in echo formation (and therefore measurement) along a particular line or axis [46]. The measurement line of the transmitted ultrasound wave is referred to as a scan line, and is shown in Figure 2.1. The scan line is therefore a summation of the echoes from reflection source along the ultrasound wave path and results in the acquisition of an ultrasound RF signal, as shown in Figure 2.2. From this ultrasound RF signal the most basic ultrasound image measurement is obtained: A-mode (amplitude mode). The different ultrasound image modalities are derived from combination of these A-mode measurements. Two different imaging modalities are used in this thesis: M-mode (motion mode) and B-mode (brightness mode). 


\section{Lateral Direction}

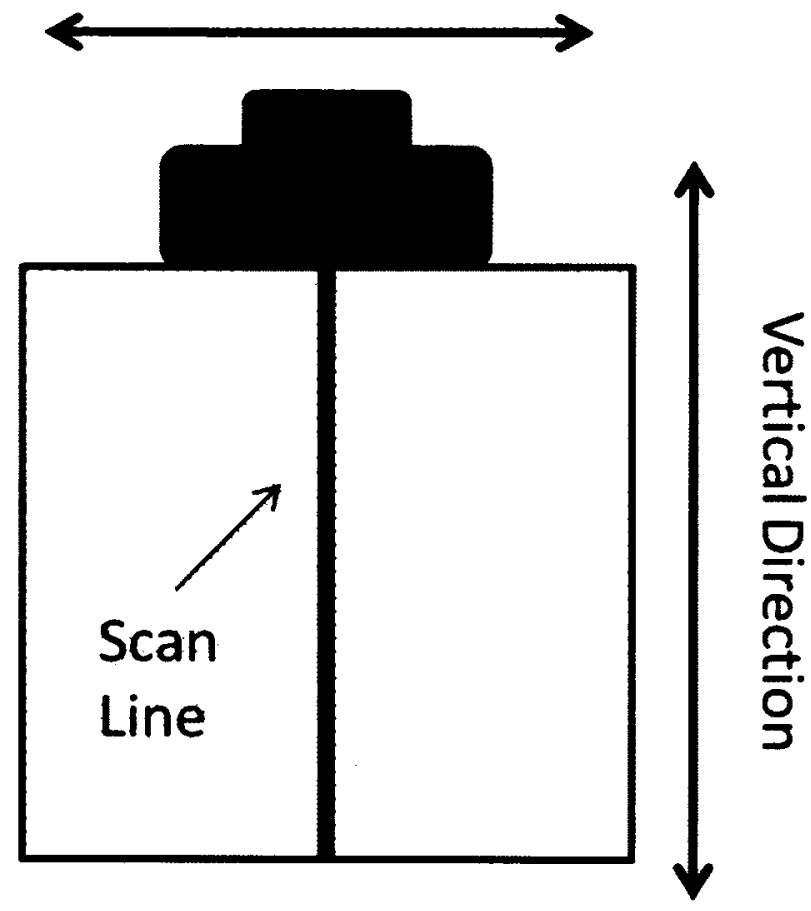

Figure 2.1 Ultrasound imaging model shows ultrasound scan line, as well as directional definitions.

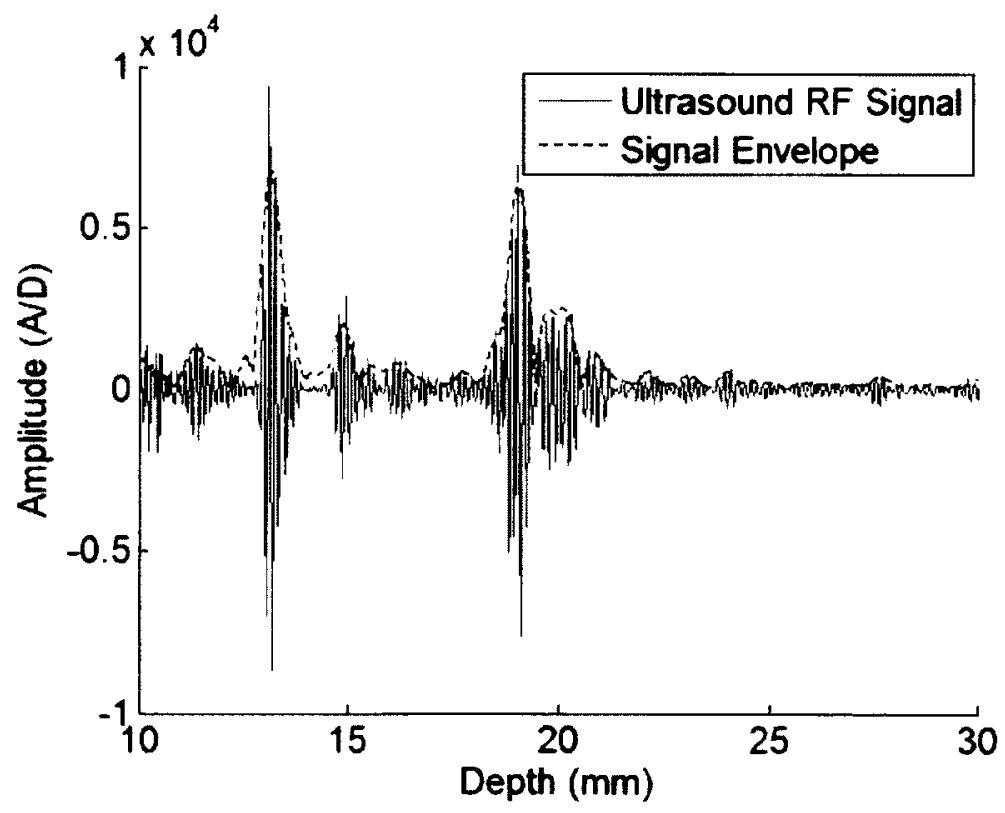

Figure 2.2 Example of ultrasound RF signal and A-mode envelope 


\subsubsection{A-Mode}

The A-mode signal is generated from the envelope of the ultrasound RF signal as shown in Figure 2.2. In A-mode measurement a single transducer is set to transmit on a repetitive basis [47]. The repetition rate is set low enough to ensure that echoes from the previous transmission will not be received, and high enough to capture any motion of interest.

\subsubsection{M-Mode}

In M-mode measurements a set of active transducer is used to measure several scan lines at the same location to generate a time series data set. The M-mode image is a sequence of Amode images captured at the same location, and provides the ability to measure the vertical tissue motion that occurs along the scan line [47]. The vertical direction is defined as the direction parallel to the ultrasound wave propagation direction, as shown in Figure 2.1. In M-mode measurements, a single scan line is referred to as a frame of data.

An example of M-mode measurements is shown in Figure 2.3. In this M-mode image, the brighter colors indicate location of echoes with large amplitude. The period from approximately $0-1.5 \mathrm{~s}$ and $4.5-5.5 \mathrm{~s}$ are regions with no motion. The activity observed between 1.5-4.5s show the vertical displacement (tissue motion parallel to the propagation direction of ultrasound) observed in the measured object. 


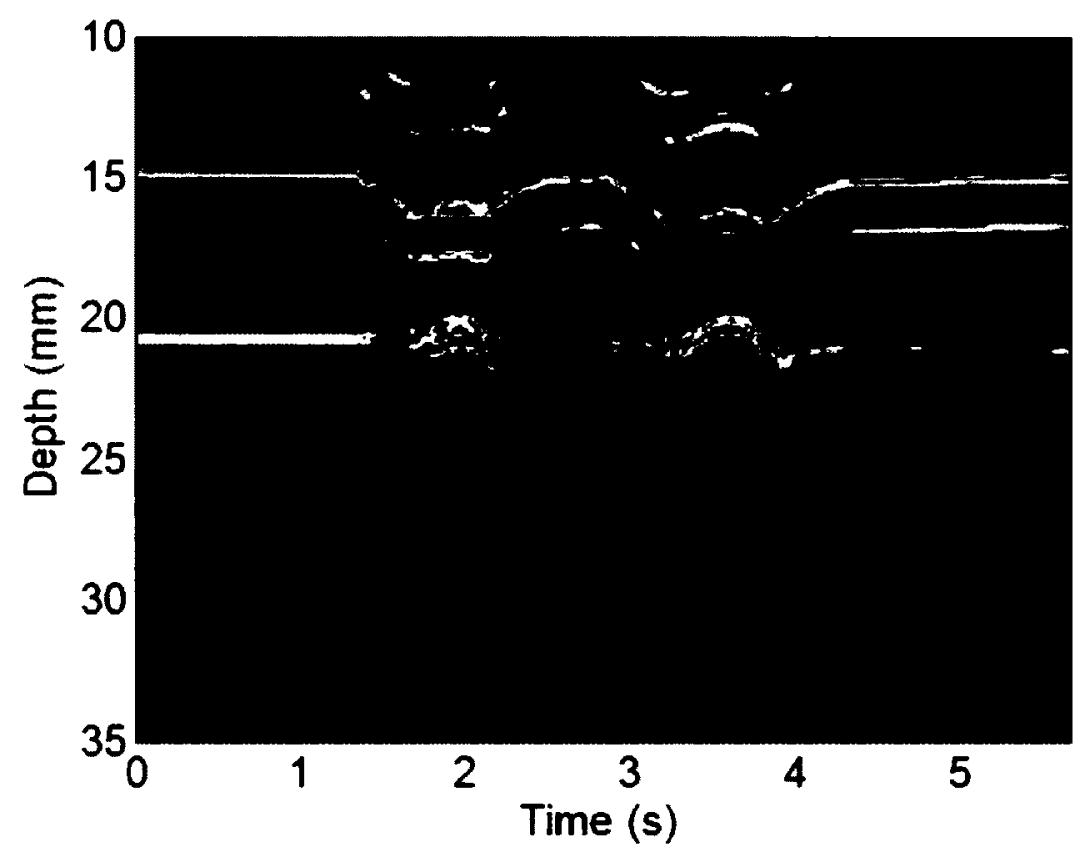

Figure 2.3 Example of M-mode image

\subsubsection{B-mode}

In B-mode imaging, A-mode scan lines from different ultrasonic transducers are combined to form a 2-D image. Compared to M-mode imaging, B-mode imaging uses electrical switching to activate sets of transducers to scan a larger area. This provides the capability to measure the internal structure of the object over a defined area. The area is defined by the arrangement of the transducers within the ultrasound probe. The most common probe shapes are linear, curvilinear, trapezoidal, sector, and radial probes [45]

An example of a B-mode image is shown in Figure 2.4. In this Figure, the colors indicate the amplitude of the received ultrasound echo. The large echoes typically occur at interfaces between different media due to difference in acoustic properties [44]. The reflection echoes generated at these interfaces allow us to discern the internal structure of the object. 


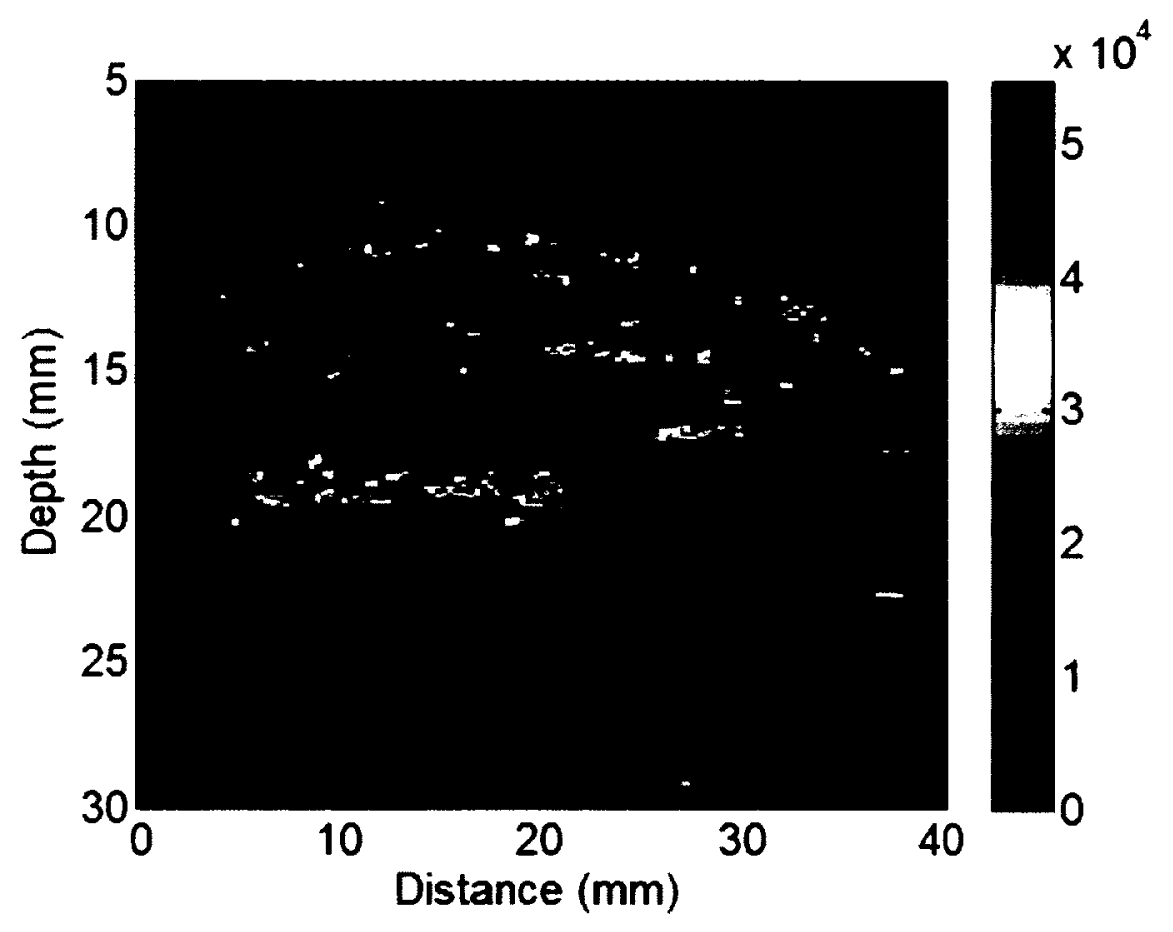

Figure 2.4 Example B-mode Image

\subsubsection{Tissue Vertical Displacement Estimation}

The tissue vertical displacement within the measurement object can be estimated from ultrasound measurements based on the changes in the measured ultrasound RF signal between different measurement frames. This displacement can be estimated from both B-mode and Mmode measurement frames. In this thesis, the displacement measurements are acquired in $\mathrm{M}$ mode because of its high frame rate. As mentioned above, M-mode measurements use a set of active transducers to measure a scan line of data per measurement frame. This allows the estimation of vertical displacement from the ultrasound measurements. In the estimated vertical displacements a positive displacement corresponds to motion away from the ultrasound probe where the distance between the probe and object increases, while negative displacement indicates motion towards the ultrasound probe.

Two different methods of estimating the vertical displacement from $M$-mode measurements are used in this thesis. The first method is cross-correlation which obtains the 
vertical displacement directly from the measured ultrasonic radio frequency (RF) signal. The other is the quadrature detection technique which uses the phase information of the RF signals and determines the displacement from changes in the phase. The basic principles of the two methods are discussed below.

\subsubsection{Cross-Correlation}

The cross-correlation method utilizes template matching to estimate the displacement directly from the RF signals. It has been used in ultrasound muscle monitoring research for vertical displacement estimation in muscles during dynamic contractions [8].

The cross-correlation method provides the ability to estimate the displacement of a specific location within the measured object. The cross-correlation template is generated from a subset of the ultrasound RF signal that contains the location of interest. The cross-correlation is then used to determine the movement of the template between measurements from different scan lines. The vertical displacement is estimated based on the movement of the template.

The length of the cross-correlation template affects the accuracy and spatial resolution of the displacement estimate. A longer template captures more information about the structure of ultrasonic echoes at the region of interest. This reduces the chance of coincidental matches with different regions that result in displacement estimation errors. However, a longer template reduces the spatial resolution of the displacement estimate. The cross-correlation method is able to detect movement of the entire template but has difficulties with detection of displacement within subsections of the template. Compared to a shorter template, a larger template requires a larger movement across the measured depth to detect the activity. Therefore, while a larger cross-correlation template increases the accuracy of the estimated displacement, it degrades the spatial resolution of cross-correlation method. 


\subsubsection{Quadrature Detection}

The quadrature detection technique $[4,36,37,48-57]$ estimates the phase change of the ultrasound RF signal. Based on the change in the phase between consecutive measurement frames it then estimates the displacement.

The received ultrasound signal can be modelled by

$$
s_{r}(n)=A(n) \cos \left(\omega_{c}(n) \cdot n T_{s}+\phi(n)\right)
$$

where $s_{r}(n)$ is the received signal, $A(n)$ is the received amplitude, $\omega_{c}(n)$ is the transmission ultrasound frequency, $n$ is the sample number along the depth direction, $T_{s}$ is the sampling rate, and $\phi(n)$ is the phase change due to movement of the scatterers within the medium [48]. During the demodulation process in quadrature detection, the received signal is multiplied by a reference signal given by

$$
S_{r e f}(n)=e^{-j \omega_{d e m} n T_{s}}
$$

where $\omega_{\text {dem }}$ is the demodulation frequency. The demodulation process to extract the inphase and quadrature signals are given by

$$
\begin{aligned}
I(n) & =L P F\left\{s_{r}(n) \cdot \cos \left(\omega_{d e m} n T_{s}\right)\right\} \\
& =\frac{1}{2} A(n) \cos \left[\Delta \omega(n) \cdot n T_{s}+\phi(n)\right] \\
Q(n) & =L P F\left\{s_{r}(n) \cdot\left[-\sin \left(\omega_{d e m} n T_{s}\right)\right]\right\} \\
& =\frac{1}{2} A(n) \sin \left[\Delta \omega(n) \cdot n T_{s}+\phi(n)\right]
\end{aligned}
$$

where $I(n)$ and $Q(n)$ are the in-phase and quadrature signals respectively, $L P F$ indicates low pass filtering, and $\Delta \omega(n)=\omega_{c}(n)-\omega_{d e m}$ is the change in frequency. The complex baseband is therefore given by 


$$
u(n)=I(n)+j Q(n)
$$

The phase can be extracted by

$$
\angle u(n)=\Delta \omega(n) \cdot n T_{s}+\phi(n)
$$

where $\angle$ indicates the phase angle. The envelope can be calculated from

$$
U(n)=\|I(n)+j Q(n)\|
$$

where $\|\cdot\|$ denotes the Euclidean norm. The quadrature detection process can therefore summarized by

$$
u(n, m)=L P F\left\{s_{r}(n, m) e^{-j 2 \pi f_{d e m} n T_{s}}\right\}
$$

where $m$ is the frame number. To calculate the displacement from the phase information, we first define the two-dimension cross-correlation function given by [56]

$$
r_{n, m}(N, M) \equiv u(n, m) \cdot u^{*}(n+N, m+M)
$$

The instantaneous displacement between consecutive frames is therefore given by [55]

$$
\Delta d(n, m)=\frac{c}{4 \pi \bar{f}_{c}(n, m)}<r_{n, m}(0,1)
$$

where $c$ is the speed of the ultrasound in the medium, $\bar{f}_{c}(n, m)$ is the estimated mean center. The displacement is therefore the accumulation of the instantaneous displacements between the preceding frames and is given by

$$
d_{z}(n, m)=d_{z}(n, m-1)+\Delta d(n, m), \text { where } d_{z}(n, 0)=0
$$

The estimated mean center frequency $\bar{f}_{c}(n, m)$ of the transmitted signal in 2.10 is given by [57]

$$
\bar{f}_{c}(n, m)=f_{d e m}-\frac{\angle r_{n, m}(0,1)}{2 \pi T_{s}}
$$

The quadrature detection technique is the primary method of displacement estimation used in this thesis. Compared to the cross-correlation method it may provide higher spatial 
resolution and better accuracy on displacement measurement within the tissue. Cross-correlation is able to accurately estimate the displacement of the medium boundaries from the large amplitude echoes from the medium boundaries. However, the cross-correlation method has issues with accurate displacement estimate from the smaller speckle echoes that occur within the medium. The speckle echoes are smaller in magnitude and exhibit a more complex behaviour compared to the larger boundary echoes. Therefore cross-correlation has issues with accurate template matching of speckle echo templates. The quadrature detection technique is selected due to higher spatial resolution and better performance in estimation of internal displacement.

However, the quadrature detection technique suffers from problems with phase-wrapping. As shown in Eq. 2.10, the displacement estimate is derived from the change in the phase of the ultrasound RF signal. However, the phase is restricted to values between $\pi$ and $-\pi$, which corresponds to displacement values of $\lambda / 2$ and $-\lambda / 2$, where $\lambda$ is the wavelength of the transmitted ultrasound wave. Displacements larger than these values result in wrapping of phase in the ultrasound RF signal and cannot be measured by the quadrature detection technique. Therefore, compared to the cross-correlation method the quadrature detection technique provides higher spatial resolution but can only measure instantaneous displacements between $\lambda / 2$ and $-\lambda / 2$.

\subsection{Displacement Measurement Errors}

Ultrasound probe motion is a major source of error in displacement estimation in ultrasound measurements. The movement of the ultrasound probe results in changes to the measured ultrasound RF signal between consecutive measurement frames. These signal changes are then perceived as vertical displacement by the cross-correlation or quadrature demodulation technique. The estimated displacement values due to probe motion are referred to as displacement estimation errors in this thesis. 
These displacement errors can result from two different sources: motion of the probe or motion of the measurement area. The ultrasound probe itself can move due to instabilities in the mounting apparatus that the probe is secured to. Global motion of the object can result in movement of the measurement area. Both probe movement and measurement object movement results in variations in the measurement area and subsequently errors in the estimated displacement

The motion errors can be classified into two main categories in this thesis: vertical and lateral motion errors. In the definition of these errors, it is assumed that the measured object location is static and it is the ultrasound probe that moves. The vertical and lateral directions are defined as shown in Figure 2.1.

\subsubsection{Vertical Motion}

As shown in Figure 2.1 vertical motion is movement in direction parallel to the ultrasound beam. The vertical motion results in a shift in the measured ultrasound RF signal. This results in a displacement error value that affects the vertical displacement estimate at all depths. This is a significant error source in ultrasonic in vivo dynamic contraction measurements due to vertical motion of the measurement area. A bone echo subtraction method has been proposed to mitigate or remove the estimated displacement errors due to vertical motion [58].

\subsubsection{Lateral Motion}

Lateral motion is movement in direction perpendicular to the ultrasound beam, as shown in Figure 2.1. This lateral movement results in changes measured ultrasound RF signal due to a shift in the measurement area. This results in displacement estimation error. The primary purpose of this thesis is to investigate the effect of the displacement estimation errors introduced 
by the lateral motion. While the primary subject of the thesis is lateral motion of the ultrasound probe, lateral motion can also occur within the measured object. For example, during muscle contraction the shortening of the muscle fiber results in both vertical and lateral movement of the fiber. Therefore, even in an ideal scenario with no probe motion the ultrasound measurement of internal vertical displacement of the muscle must also consider the effect of the lateral muscle movement.

\subsubsection{Rotation and Shear Motion}

Another application of ultrasound imaging is in the field of elastography to determine the elastic properties of the object. In elastography, a compression force is applied to the object and the resulting internal displacement is used to estimate the elastic property of the object. This elasticity value can be used in tissue classification and tumor detection.

In ultrasound elastography the ultrasound probe is often used as the compression source $[59,60]$. The force applied to the probe may not be even and can result in probe rotation or shearing of the measured object. Research has been performed to investigate the effect of probe rotation or measurement object shearing in elastography $[59,60]$. 


\section{Focused Ultrasound Beam Width Measurement}

In this chapter, the beam with profile of the clinical ultrasound system used in the thesis is estimated. The profile of the beam width determines the size of the measurement area and is an important parameter for the numerical simulation (presented in Chapter 4). The clinical ultrasound system (model PICUS, ESAOTE, Maastricht, Holland) with a linear array probe (model L10-5, ESAOTE, Maastricht, Holland) is used for the ultrasound measurements. The method and experimental setup to measure the beam width profile is first presented. Afterwards, the experimental results and beam profile are discussed.

\subsection{Measurement Procedure}

The size of the measurement area is determined by scanning the probe across a point reflector that is much smaller in size compared to the ultrasound beam width. The point reflector only produces a reflection echo while it is within the transmitted ultrasound beam. By scanning the probe across the point reflector and measuring the distance where an echo is observed we can determine the beam width of the ultrasound source. A model of the procedure is shown in Figure 3.1 . 

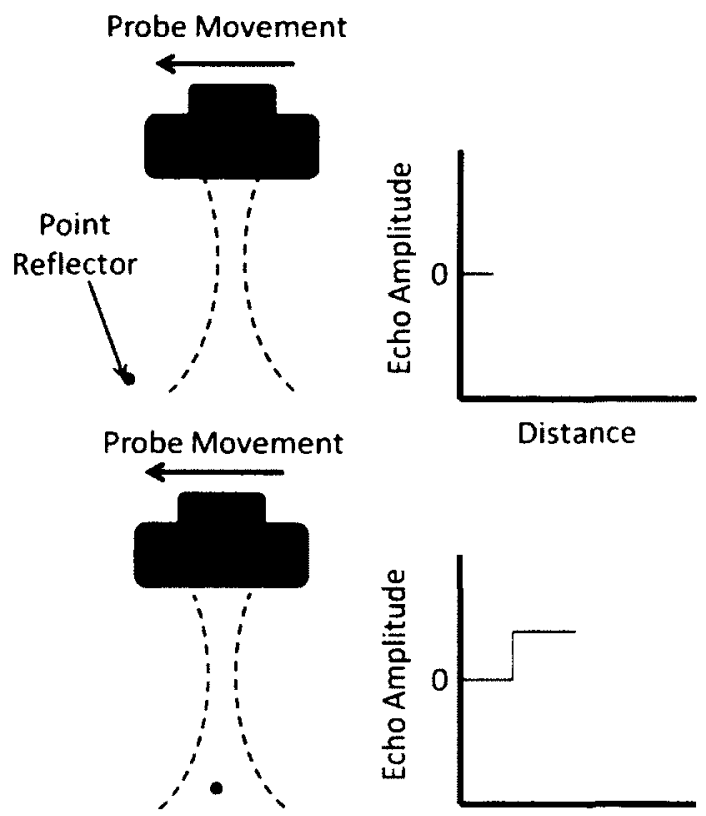

Distance
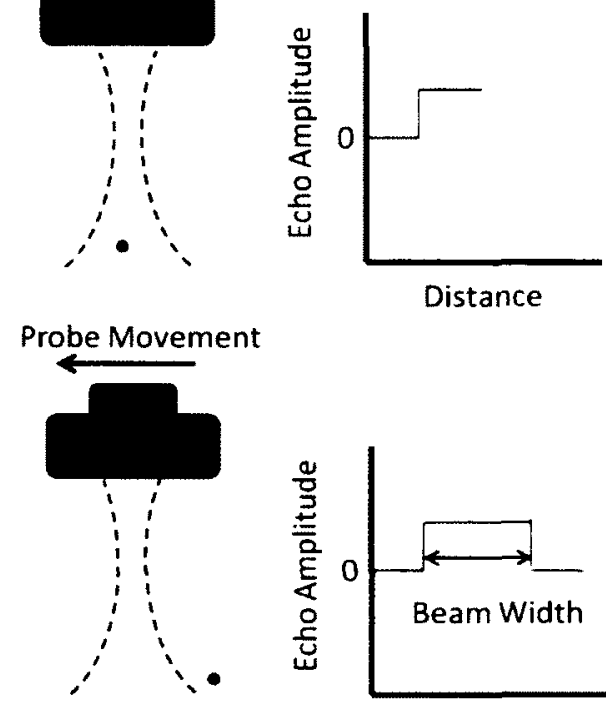

Distance

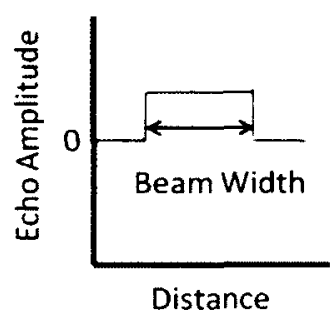

Figure 3.1 Beam width idealized measurement model. The ultrasound probe is modelled by the blue rectangular object, and the point reflector by the red circle. The dotted lines mark the beam width of the transmitted ultrasound beam.

However, a point reflector returns a weak echo that is similar in magnitude to the measurement noise floor. Therefore, a sharp razor blade is used as a line reflector instead. The razor is attached to a Plexiglas plate such that it stands perpendicular to the probe translation direction. When the razor is perpendicular to the translation direction of a rectangular transducer, the transducer views the razor by the thinnest side. Since the width of the razor along this side is much smaller than the size of the ultrasound beam width, it can be used as an approximation to a point reflector along the translation direction. 
The experiment setup is shown in Figure 3.2. The razor is attached to a Plexiglas cube with epoxy resin. The razor and Plexiglas cube are placed into a water bath such that the razor is perpendicular to the container bottom. The water bath serves as an ultrasound transmission medium and allows the ultrasound probe to measure the reflected echo at a distance from the razor. The ultrasound probe is secured to a metal holster which is attached to a mechanical stage (model BiSlide, VELMEX Inc., Bloomfield, NY). The mechanical stage is driven by a stepping motor that is able to move the probe at a desired constant velocity. While, the probe is scanned across the razor edge M-mode measurements are conducted. From the measured results the probe beam width can be calculated.

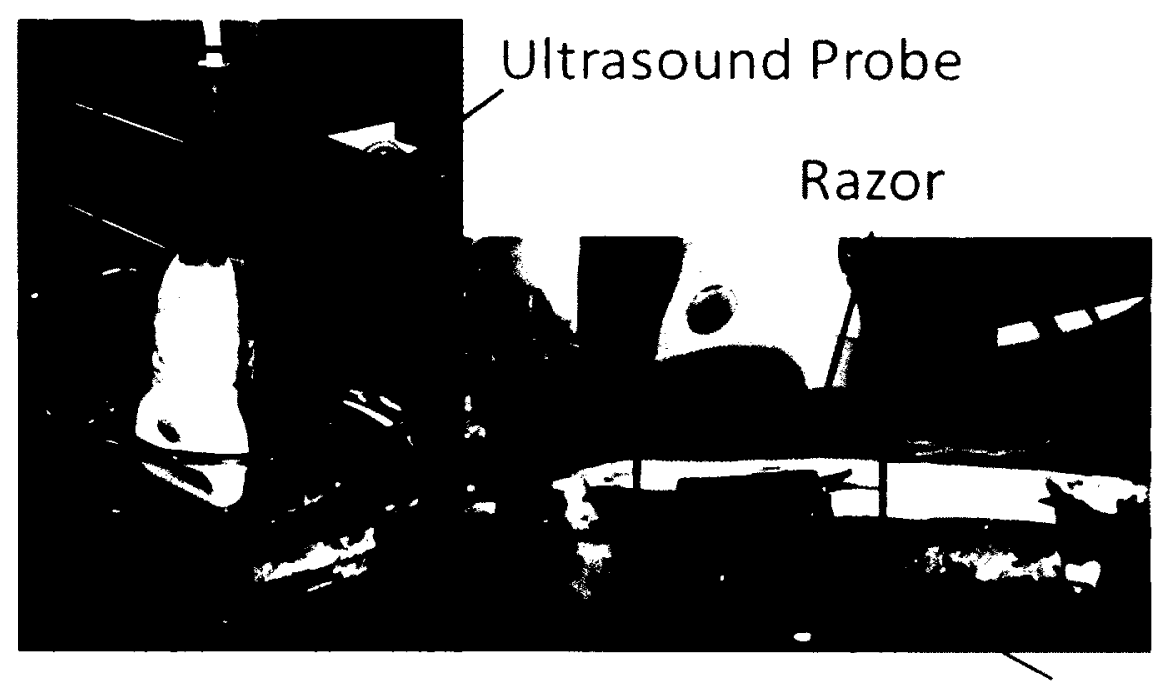

Figure 3.2 Experiment setup for beam width measurement. In this setup, the razor is situated to measure the beam width in the mechanical focusing direction. 
The measurement was repeated at different depths. This was to determine the changes of the beam width as the transmitted beam travelled away from the probe. It was also used to determine the location of a focal distance for the M-mode measurements. The focal distance is the distance from the probe where the transmitted ultrasound has the smallest beam width.

The beam width experiment is used to calculate the probe beam width in both the electrical and mechanical focusing direction. The probe is a linear array transducer that consists of 127 individual transducers oriented in a line. The activation of the individual transducers is controlled by electrical switching. The ultrasound beam can be focused based on the switching order and time delay of the transducers in the array line direction. The ultrasound beam focusing by electrical switching is referred to as the electrical focusing. Conversely, in the direction normal to the array line, the ultrasound probe lens is shaped to focus ultrasound beam at a fixed depth. The focusing in this direction is referred to as mechanical focusing as the focal width and distance are controlled by the lens dimension. To measure the beam width the razor is placed in a direction perpendicular to the intended focal direction.

\subsection{Experimental Results}

The amplitude of the razor reflection echo at different distances from the probe is shown in Figure 3.3 and Figure 3.4, respectively, for the electrical and mechanical focusing direction. The razor reflection amplitude is determined by the magnitude of the razor reflection in the received ultrasound RF signal. The amplitude profile of the beam shows a Gaussian shape. Therefore, the beam width is defined as the distance between the two points where the reflection echo is half of the maximum amplitude (full-width at half maximum: FWHM). The plot of the beam width in both the electrical and mechanical focusing is shown in Figure 3.5. 


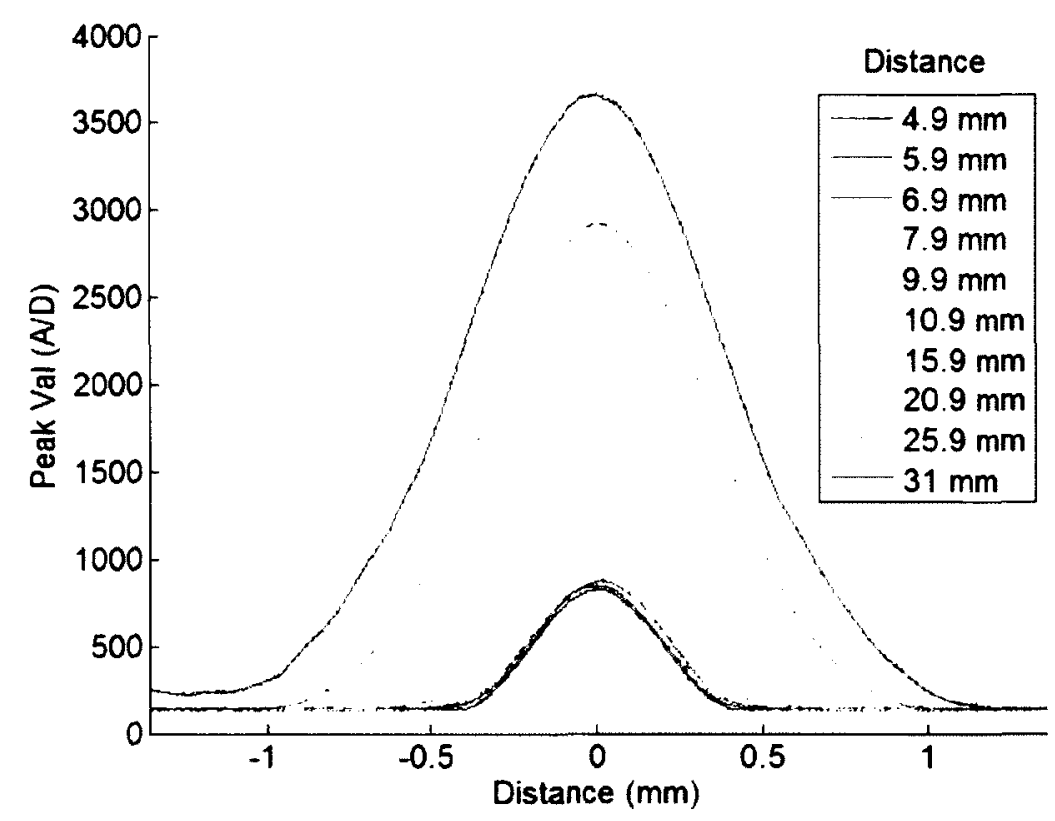

Figure 3.3 Reflection echo profile from razor in electrical focusing direction at different measurement distances.

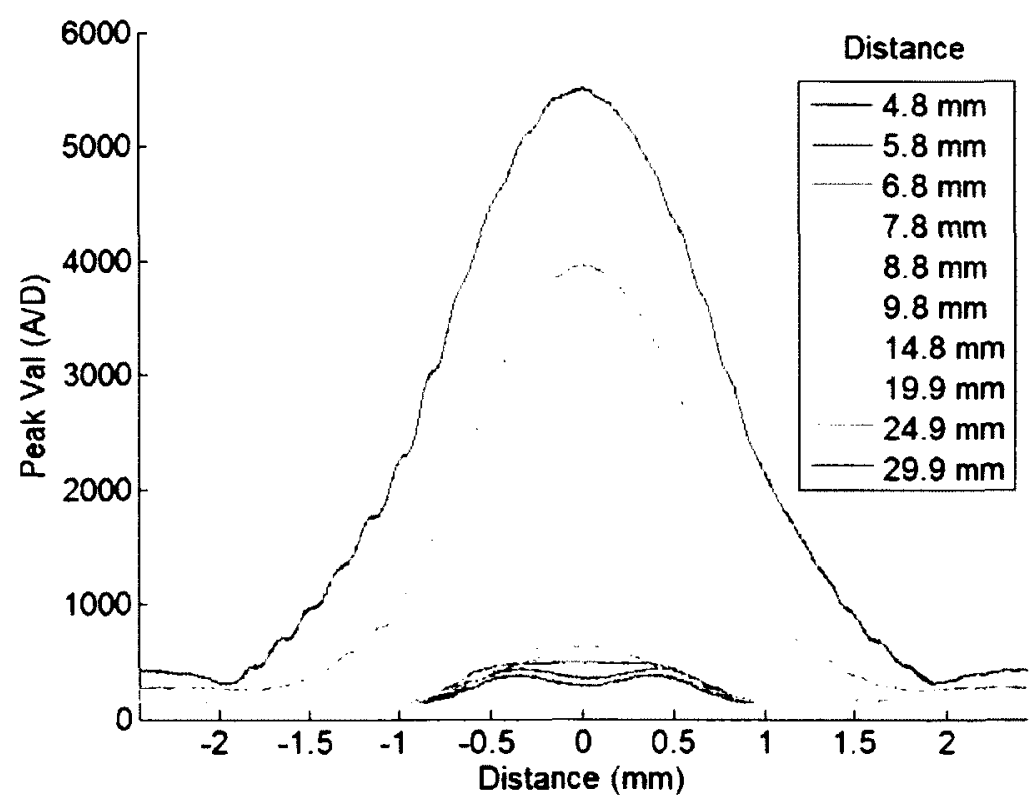

Figure 3.4 Reflection echo profile from razor in mechanical focusing direction at different measurement distances. 


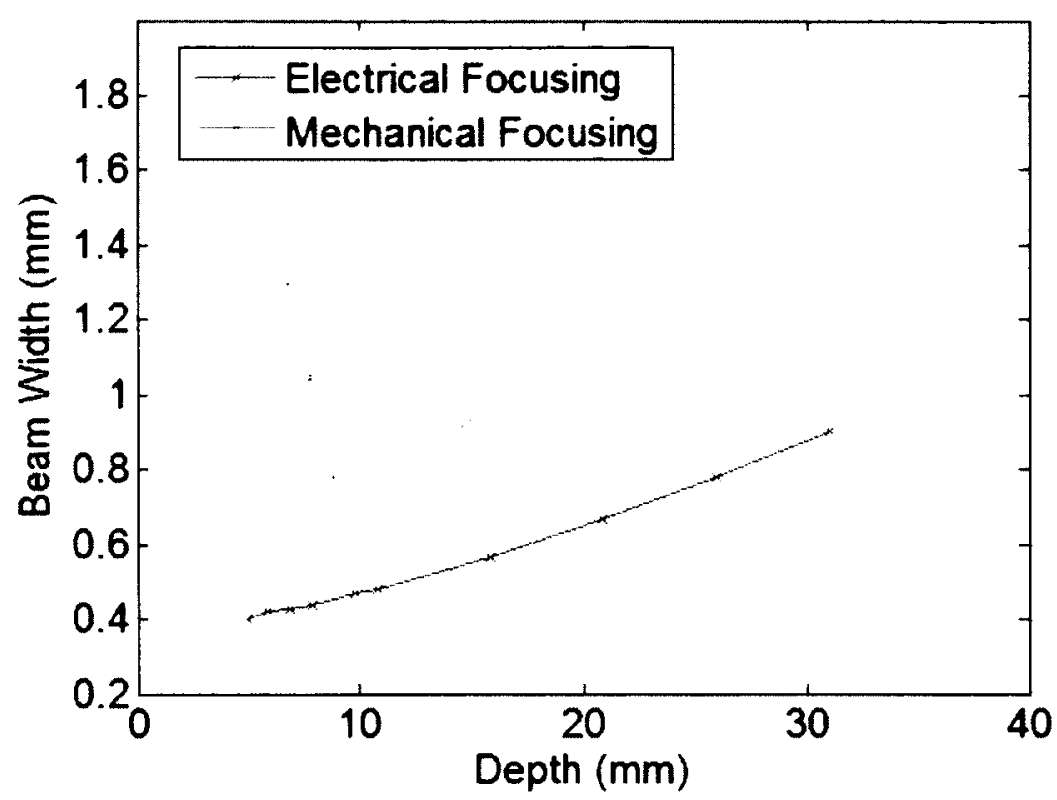

Figure 3.5 Transmitted ultrasound beam width in electrical and mechanical focusing directions.

From the dip in the beam width in the mechanical focusing direction of the ultrasound probe in Figure 3.5 there appears to be a focal point at around $10 \mathrm{~mm}$ distance from the probe. The focal distance is the location where the ultrasound beam width is the smallest; therefore the transmitted ultrasound energy density is at the highest. As the distance from the focal distance increases the energy is more dispersed and therefore the beam width increases. In the electrical focusing direction, the beam width slightly increases from $0.4 \mathrm{~mm}$ to $1 \mathrm{~mm}$ with depth because of dynamic beam focusing function equipped with the imaging system used [61].

From examination of the razor echo amplitude profiles at different distances in Figure 3.3 and Figure 3.4 the beam profile for most distances appear to be a Gaussian shaped curve. The bell shaped curve shows that the most of the energy is centered along the transmission path, but due to the unguided transmission energy also leaks away from the center. There are some exceptions to the Gaussian shape curve; in particular the plots of the razor echo amplitude for the 
mechanical focusing direction when the razor is close to the probe. An example is shown in Figure 3.6.

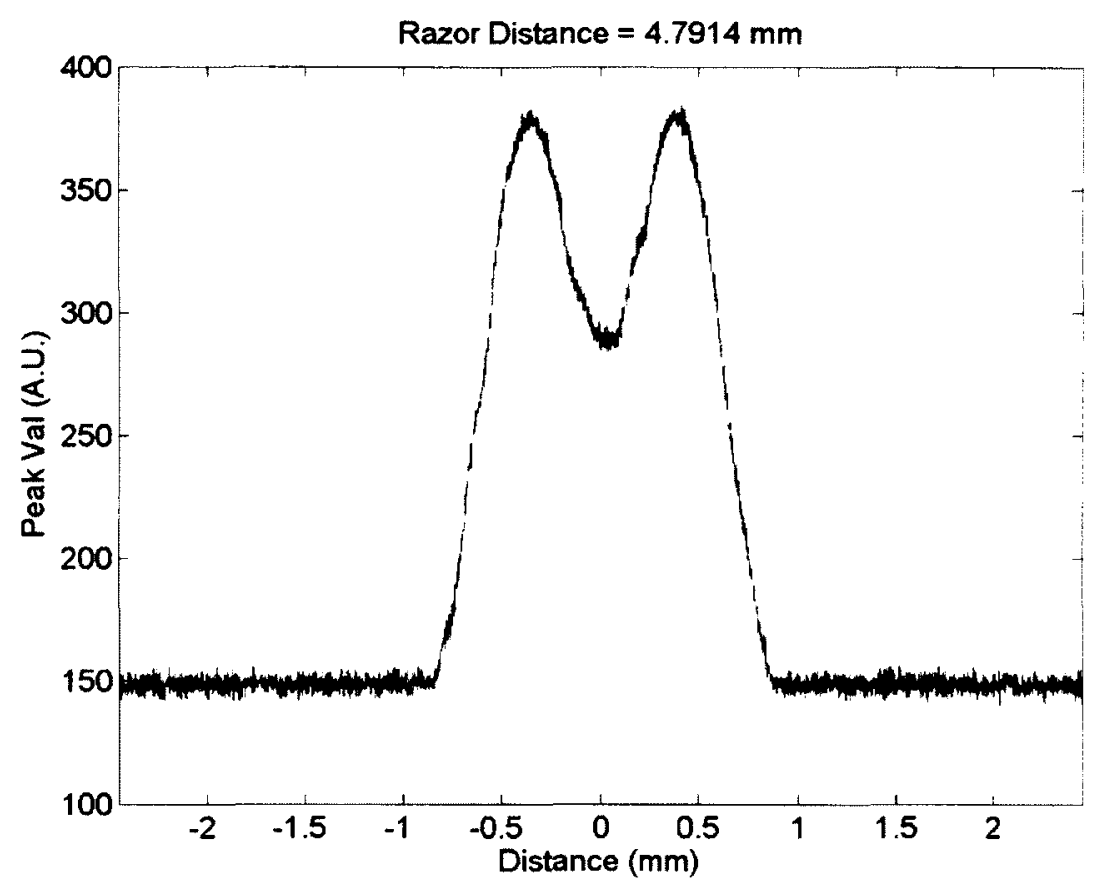

Figure 3.6 Razor Echo Amplitude Profile, Mechanical Focusing

In the plot in Figure 3.6 of the razor echo amplitude from the mechanical focusing experiment instead of a Gaussian shaped curve a dip is observed in the center of the profile. This is due to the diffraction of ultrasound in the near field (Fresnel zone) [62]. 


\section{Effect of Lateral Motion}

In this chapter, the effects of a probe lateral motion and random noise in ultrasonic RF signal on the phase estimation accuracy are investigated through numerical and tissue mimicking phantom simulations. Stability and accuracy of the clinical ultrasound imaging system and experimental setup used in this thesis were first examined for vertical displacement estimation. Afterwards, analysis of lateral motion effect through numerical simulations is presented along with the approach used to quantify the observed phase estimation error. Both the models used in the numerical simulation and simulation results are discussed. Finally, experimental results using tissue mimicking phantoms are presented for model verification of the numerical simulation. In addition, a moving average filter is tested in order to reduce the phase estimation error caused by random noise.

\subsection{System Stability and Accuracy}

Before the effect of lateral motion can be considered, the noise present in the experimental conditions must be examined. Measurements inherently deal with a variety of noise sources, such as random electrical noise, environmental noise, and random movement caused by vibrations within the experimental hardware setup. These noise sources result in an error in the displacement estimate.

In this section the procedure used to measure the inherent error in the experimental setup is introduced. Afterwards, the analysis of noise effect on phase estimate accuracy is presented. 


\subsubsection{System Stability Experimental Procedure}

The experiment setup is shown in Figure 4.1. A 3-W\% agar phantom containing carbon particles as ultrasound scatterers [58] is placed under the ultrasound probe and M-mode data is acquired for $5.5 \mathrm{~s}$ at the frame rate of $980 \mathrm{~Hz}$ with the sampling frequency of $33.3 \mathrm{MHz}$. Therefore, a total of 5550 ultrasonic RF signals are acquired. No motion is applied to the ultrasound probe or phantom during the acquisition. Thus, any temporal variation in the measured ultrasound RF signal is therefore due to noise source in the measurement setup.

To limit the influence that a specific phantom may have on the results, the experiment is repeated with another $3-\mathrm{W} \%$ agar phantom. This is to ensure that observed error behaviour is not due to specific phantom defects or individual structural properties. This also assists in generalization of the experimental setup error estimates.

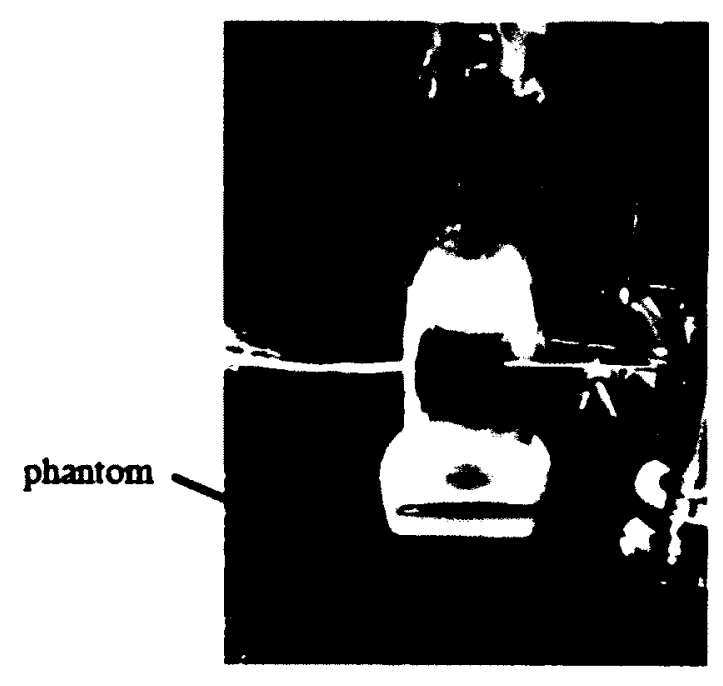

Figure 4.1 Experiment Stability Setup

\subsubsection{Experimental Results}

The quadrature detection technique is applied to estimate the displacement at each depth from phase changes between two consecutive ultrasound RF signals acquired. The stability or 
accuracy of the system is determined from the standard deviation of the estimated displacement at each depth over the entire $5.5 \mathrm{~s}$ measurement period. The standard deviation provides a quantification of the variable error in the displacement estimates due to noise. As mentioned before, since the measured object is at rest any estimated displacement values are errors due to noise.

The standard deviations of the estimated error from the two phantom measurements are shown in Figure 4.2 and Figure 4.3. The measurements from the two phantoms both produce similar values of the estimated displacement standard deviation. In both plots the majority of the standard deviation values across the depth are below $1 \mu \mathrm{m}$. However, at several depths, spikes in the estimated displacement standard deviation occur that result in values above $1 \mu \mathrm{m}$. Both Figure 4.2 and Figure 4.3 have an error floor of approximately $0.2 \mu \mathrm{m}$ which provides an estimate of the maximum accuracy of the measurement setup.

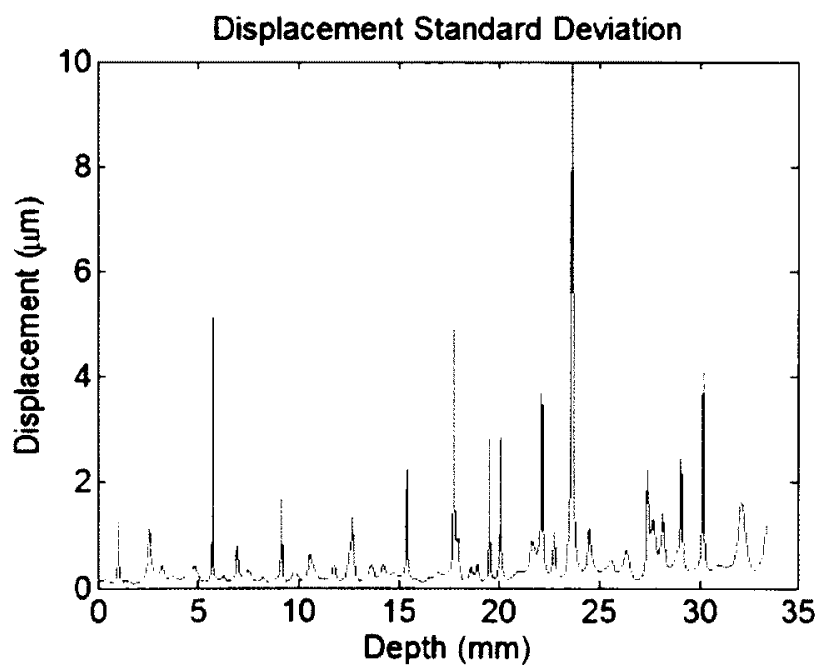

Figure 4.2 Experimental Setup Measurement Error, Phantom \#1 Measurement 


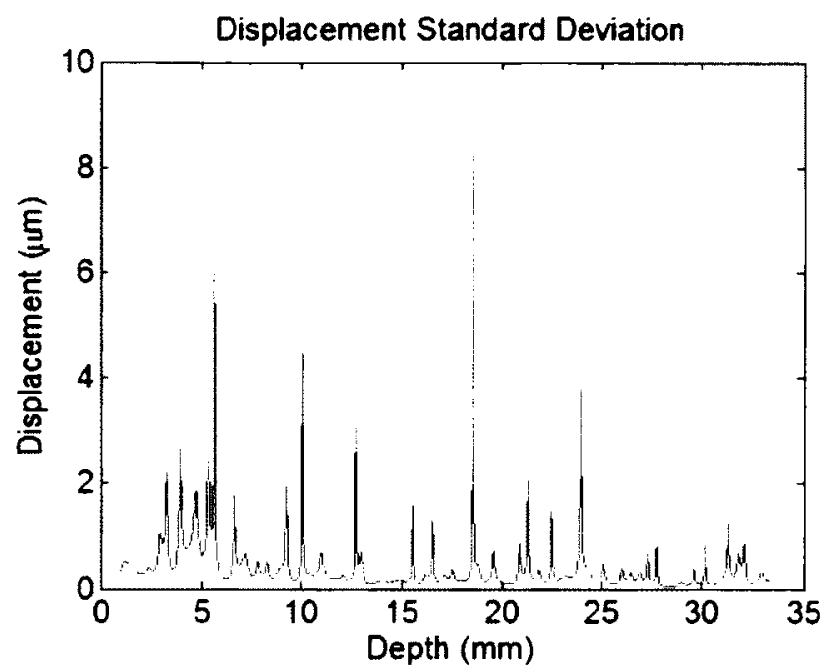

Figure 4.3 Experimental Setup Measurement Error, Phantom \#2 Measurement

\subsection{Lateral Motion}

As described in the previous chapters, the lateral motion is due to undesired relative lateral movement between the ultrasound probe and measurement subject. The lateral motion model is shown in Figure 4.4, and defines the lateral and vertical directions used in the thesis. The lateral motion distance is defined as the lateral distance travelled between two measurement frames. This lateral motion causes a change in the field-of-view ( $F o V)$ of the probe and results in differences between the received echoes in the two measurement frames. 


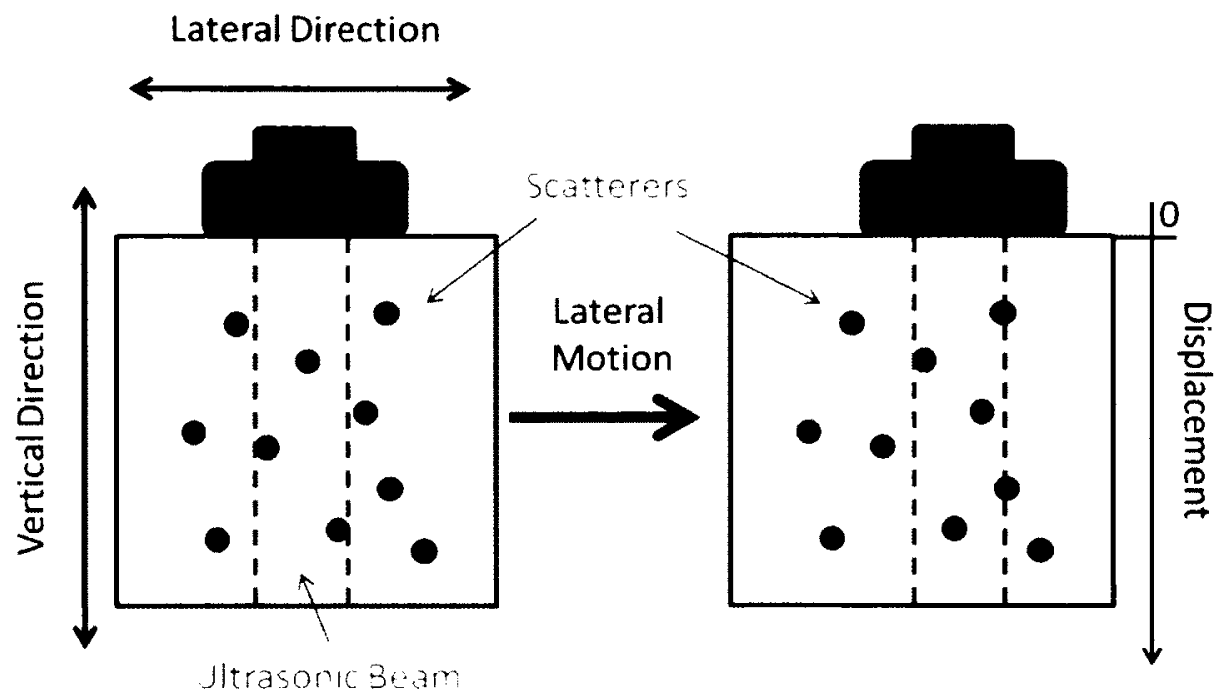

(a)

(b)

Figure 4.4 Measurement configuration and direction. The probe location and measurement area before (a) and after (b) lateral motion.

One application of ultrasound muscle monitoring is measurement of the vertical displacement (tissue displacement along (or parallel to) the propagation direction of ultrasonic beam) within the muscle. The method used to ascertain the displacement is the speckle echo tracking method by estimating the phase of ultrasonic RF signals using the quadrature demodulation technique, as described in Chapter 2. This technique determines the tissue displacement based on the estimated phase information of the ultrasound RF signal. If there is no tissue vertical displacement, there should be no temporal variation in the phase of the acquired ultrasound RF signal. Therefore, we wish to examine the effect of the lateral motion based on the estimated phase information of the ultrasound RF signal.

In addition, we also want to examine the effect of the lateral motion at different signal-tonoise-ratios (SNRs) on the received ultrasound RF signal. The SNR is a measure of the quality of the received echo, with large SNR values typically corresponding to strong echoes at medium boundaries. As the ultrasound wave propagates through the medium, the observed echoes are 
from intra-medium scatterers. These echoes are weaker in magnitude relative to boundary echoes and are harder to distinguish from noise. Therefore, these scatter echoes typically have low SNR. Through determination of the relationship between SNR and phase estimate error we can determine the minimum SNR required to ensure accurate measurement.

\subsection{Numerical Simulation}

Numerical simulations are used for initial analysis of the noise due to lateral probe motion. These simulations provide us with complete control over all noise parameters, and enable us to ensure that the observed behaviour is due to lateral motion and not from other sources.

\subsubsection{Mathematical Model}

In order to investigate the effects of lateral probe motion, a two dimensional model is required since the ultrasound beam has a width and depth in a medium (or subject). To begin with, however, we present the theory for the one dimension ultrasound model. Afterwards, we demonstrate how this model can be extended to a two-dimensional model.

The one dimension model assumes that the transmitted ultrasound signal is an infinitely narrow beam. A Gaussian pulse-echo model [63] is used to model the transmitted ultrasound pulse signal, and is given by

$$
P(t)=e^{-\beta \cdot t^{2}} \cdot \cos \left(\omega_{c} t\right)
$$

where $t$ is the fast time, or time after pulse transmission, $\beta$ is the bandwidth factor, and $\omega_{c}$ is the center frequency of the transmitted ultrasonic pulse signal. As the ultrasonic signal travels through the medium, it encounters scatterers within the medium. These scatterers cause scattering (or speckle) echoes to form as the transmitted ultrasound signal arrives at their location. A single scattering echo can be modelled by 


$$
\begin{aligned}
S_{k}\left(\tau_{k} ; t\right) & =\alpha_{k} P\left(t-\tau_{k}\right) \\
& =\alpha_{k} e^{-\beta\left(t-\tau_{k}\right)^{2}} \cdot \cos \left(\omega_{c} t-\omega_{c} \tau_{k}\right), k=1,2,3 \ldots L
\end{aligned}
$$

where $S_{k}\left(\tau_{k} ; t\right)$ is the echo from the $k^{t h}$ scatterer, $\alpha_{k}$ is the amplitude factor and $\tau_{k}$ is the time required for the transmitted ultrasound pulse to arrive at the $k^{\text {th }}$ scatterer $[63,64] . L$ is the total number of scatterers within the ultrasound beam. The time delay $\tau_{k}$ results in a phase shift in the received scattering echo. Therefore, Eq. 4.2 can be rewritten as

$$
S_{k}\left(\tau_{k} ; t\right)=A_{k}(t) \cdot \cos \left(\omega_{c} t-\phi_{k}\right)
$$

where $A_{k}(t)=\alpha_{k} e^{-\beta\left(t-\tau_{k}\right)^{2}}$ and $\phi_{k}=\omega_{c} \tau_{k}$. We can see that $A_{k}(t)$ and $\phi_{k}$ represents the amplitude and phase information, respectively, of the $k^{\text {th }}$ echo. The time delay $\tau_{k}$ is dependent on the location of the scatterer along with the ultrasonic propagation velocity and is given by

$$
\tau_{k}=\frac{2 z_{k}}{c}
$$

where $z_{k}$ is the distance from the scatterer to the probe along the vertical axis and $c$ is the speed of the ultrasound in the medium. While Eq. 4.3 is valid for a simplified one-dimensional scenario we know that the ultrasonic beam has a finite width. We can therefore extend Eq. 4.3 to a two-dimensional case by including the beam profile in the lateral direction. The twodimensional model and axis are shown in Figure 4.5. 


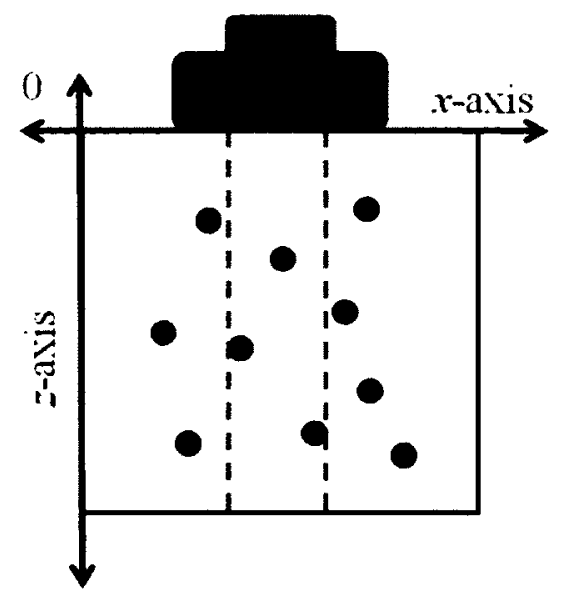

Figure 4.5 Two-dimensional ultrasound model and scatterer axis definition. The $(0,0)$ coordinate corresponds to a scatterer location in the top left corner of the medium.

A scatterering echo in the two-dimensional scenario is therefore

$$
S_{k}\left(\tau_{k}, x_{k}, x_{c} ; t\right)=W\left(x_{c}, x_{k}\right) \cdot A_{k}(t) \cdot \cos \left(\omega_{c} t-\phi_{k}\right)
$$

where $W\left(x_{c}, x_{k}\right)$ is a function that models the beam profile, and $x_{k}$ and $x_{c}$ are the location of the $k^{\text {th }}$ scatterer and ultrasonic probe center respectively along the lateral axis. From Figure 3.3, it appears that the shape of the beam profile in the lateral direction is similar to a Gaussian function. Therefore, a Gaussian function is used to model the ultrasonic beam profile. The function of the ultrasound beam profile is given by

$$
W\left(x_{c}, x_{k}\right)=e^{-\frac{4\left(x_{k}-x_{c}\right)^{2}}{x^{2}}}
$$

where $X$ is the width of the ultrasound beam. Therefore, the scattering echo from the $k^{\text {th }}$ scatterer is modelled by

$$
S_{k}\left(\tau_{k}, x_{k}, x_{c} ; t\right)=e^{-\frac{4\left(x_{k}-x_{c}\right)^{2}}{X^{2}}} \cdot A_{k}(t) \cdot \cos \left(\omega_{c} t-\phi_{k}\right)
$$


The received ultrasound echo signal is a superposition of all the scatterers within the transmitted ultrasonic beam. The received ultrasonic $\mathrm{RF}$ signal, $S_{r}(t)$, can therefore be represented as

$$
\begin{aligned}
S_{r}(t) & =\sum_{k=1}^{L} S_{k}\left(\tau_{k}, x_{k}, x_{c} ; t\right) \\
& =\sum_{k=1}^{L} e^{-\frac{4\left(x_{k}-x_{c}\right)^{2}}{X^{2}}} \cdot A_{k}(t) \cdot \cos \left(\omega_{c} t-\phi_{k}\right),
\end{aligned}
$$

where $L$ is the total number of scatterers within the ultrasonic beam. By converting Eq. 4.8 into a more practical discrete form we get

$$
S_{r}(n)=\sum_{k=1}^{L} e^{-\frac{4\left(x_{k}-x_{c}\right)^{2}}{x^{2}}} \cdot A_{k}\left(n \cdot T_{s}\right) \cdot \cos \left(2 \pi f_{c} \cdot n \cdot T_{s}-\phi_{k}\right)
$$

where $f_{c}$ is the center frequency in $\mathrm{Hz}, T_{s}$ is the sampling period, and $n$ is the sample index corresponding to the depth. The received signal is a sum of pulsed sinusoids where the distance measure is encoded in the phase information of the signal. To extract the phase information the quadrature demodulation technique is applied.

\subsubsection{Phase Estimation Parameterization}

In order to analyze the effect of lateral motion on the phase estimation of ultrasonic RF signals, a parameter to quantify the observed error is necessary. The parameter chosen in this thesis is the root mean square (RMS) error. The RMS of a signal $\boldsymbol{x}$ is defined as

$$
R M S=\sqrt{\frac{1}{N} \sum_{n=1}^{N} x_{n}^{2}}
$$


The RMS is a popular statistical measure for the magnitude of a varying signal, especially in situations where the signal magnitude may be positive or negative. Thus, this parameter is a good fit for evaluating phase estimation error.

\subsubsection{Noise Quantification}

It is important to determine the noise level in the ultrasound RF signal relative to the desired reflection echoes. This comparison is represented through the SNR and is an indication of the accuracy of the information estimated based on the ultrasound RF signal. To define the SNR in this thesis, the envelope $A(n)$ of the ultrasound signal amplitude is first determined, where $n=1 . . N$ is the sample number of the ultrasound signal and $N$ is the total number of samples. The sample number of the ultrasound signal may be attributed to a specific depth via the known sampling rate and predetermined ultrasound propagation velocity within a medium. As noise signals may be depth-varying, the standard deviation $\sigma(n)$ of the amplitude of ultrasonic signals at a particular sample index $n$ (or depth) is pre-determined from RF signals with no vertical motion. Then, the SNR at a particular sample $n$ is given by

$$
S N R(n)=20 \cdot \log _{10} \frac{A(n)}{2 \sigma(n)}
$$

One relationship that we wish to determine is the influence of the SNR on the phase estimation accuracy. In order to determine this we calculate the phase RMS at specific level of SNR. The process to calculate it is described below:

1. $\sigma(n)$ of the amplitude of ultrasonic signals at depth index $n$ is pre-determined from ultrasound RF signals with no vertical motion.

2. Calculate $\operatorname{SNR}(n)$ of the frame $m$ using Eq. (4.11). 
3. Determine the phase difference $\phi(n)$ between two consecutive frames $m$ and $m+1$

4. Bin the elements of $\phi(n)$ based on the value of $S N R(n)$. SNR container width of $0.1 \mathrm{~dB}$ is used.

5. Calculate the phase RMS error of each container.

\subsubsection{Parameter setting for numerical simulation}

The model described in the previous section is used to simulate received ultrasound $\mathrm{RF}$ signals reflected by scatterers in the medium. The parameter values used in the simulation are selected to match the performance of the ultrasound imaging system used for the experiments in this thesis. The center frequency is $f_{c}=5 \mathrm{MHz}$, the sampling period is $T_{s}=0.03 \mu \mathrm{s}$, and ultrasound beam width is $700 \mu \mathrm{m}$. The beam width was selected based on an average of the measured electrical focusing beam width found in the Chapter 3. The measurements performed in this thesis are on human body or tissue-mimicking phantom therefore an ultrasound propagation velocity of $c=1540 \mathrm{~m} / \mathrm{s}$ was assumed. The Gaussian pulse bandwidth factor is

chosen to be $0.64(\mathrm{MHz})^{2}$ based on previous work [65]. In order to simulate the performance of the clinical ultrasound imaging system used in this thesis, the number of simulated samples per measurement frame is set to $\mathrm{N}=1448$ samples/frame, which corresponds to a depth of $35 \mathrm{~mm}$. The scatterer amplitude factor is assumed to be unity $\left(\alpha_{k}=1, k=1,2,3 \ldots L\right)$. This is primarily for simplification purposes as the parameter of interest (phase) is amplitude independent.

The simulated propagation medium is specified to model a tissue-mimicking phantom used in the phantom simulation experiments described in Section 4.4. The simulation medium is a $35 \mathrm{~mm}$ by $35 \mathrm{~mm}$ square object with randomly uniformly distributed ultrasonic scatterers. A total of 17500 scatterers are randomly distributed to achieve an average distance of $300 \mu \mathrm{m}$ 
between scatterers. A pseudorandom number generator was used to approximate a uniform random distribution between 0 to $35 \mathrm{~mm}$. This pseudorandom number generator was used to assign a random $x$ and $z$ coordinate to each ultrasound scatterer in order to approximate a random uniformly distributed set of ultrasound scatterers.

To simulate actual received ultrasonic RF signals in which noise exists in practice, random additive white Gaussian noise (AWGN) is added to the simulated signal. The AWGN is used to model the random noise elements inherent in real measurement conditions. In particular, it is used to represent the random electrical noise along with the environmental noise observed in ultrasound measurements. AWGN is parameterized by the noise standard deviation and is set to $\sigma=0.20$ based on the noise measurement of the experimental setup obtained in Section 4.1.

The initial location of the probe was set at the center of the simulation body $\left(x_{c}=17.5\right.$ $\mathrm{mm}$ ). At this position, one frame of ultrasound RF signal is simulated. Lateral motion is then applied to the probe by moving the probe by a desired amount. In these investigations, lateral motion distances of $0 \mu \mathrm{m}, 0.1 \mu \mathrm{m}, 1 \mu \mathrm{m}, 10 \mu \mathrm{m}, 100 \mu \mathrm{m}$, and $1000 \mu \mathrm{m}$ are applied to examine a wide range of potential cases both in numerical and phantom simulations. The phase difference between the two simulated RF signals is then determined. In order to reduce the impact of a specific scatterer structure and patterns, 100 trials of the simulation are performed. The phase difference obtained from the 100 trials is used to calculate the phase RMS error and signal SNR based on the method described in Sections 4.3.2 and 4.3.3, respectively.

\subsubsection{Numerical Simulation Results}

An example of the simulated RF signal is shown in Figure 4.6 alongside a RF signal obtained with a homogenous phantom using the clinical ultrasound system. Visual observation of the signal allows a qualitative assessment of the accuracy of the simulation model. The most 
evident difference between the simulated and measured ultrasound RF signal is the smoothness of the respective signals.

The measured signal has a large number of sharp peaks that are not present in the simulated signal. This difference is likely due to a combination of the simplifications and unaccounted for factors in the simulation model. The bandwidth factor used in the numerical simulation could be different from the actual bandwidth of the clinical imaging system. This difference could result in a longer echo from the scatterer and could contribute to the smoothness of the simulated signal. These peaks in the measurement may also be due to interaction of scattering echoes from different scatterers. This complex behaviour is not included in the model used in the simulated results, and is one potential source of error.
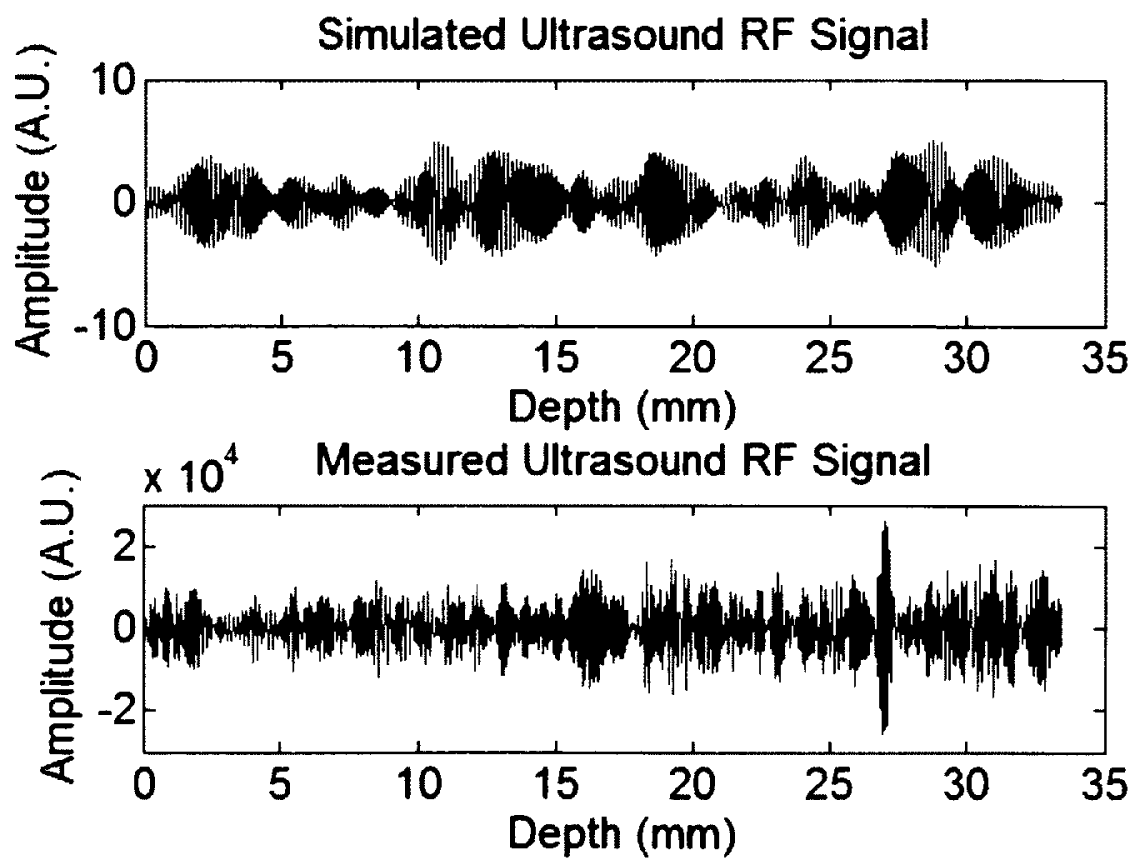

Figure 4.6 Simulated and measured ultrasound RF Signal. The simulated signal (top) and the measured signal (bottom) are shown 
To examine the accuracy of the simulated ultrasound RF signal the spectral content of the simulated signal is compared to a measured ultrasound RF signal. The ultrasound RF signal spectrums are calculated from the amplitude of the discrete Fourier transform applied to the simulated and measured ultrasound RF signal. An example of the spectral content of a simulated and measured ultrasound RF signal is shown in Figure 4.7.

We can see that majority of the spectral content of both the simulated and measured ultrasound RF signals are located at approximately $5 \mathrm{MHz}$, which is the center frequency of the simulated and clinical ultrasound system. However, the simulated ultrasound RF signal has narrower bandwidth ( $1 \mathrm{MHz}$ ) compared to the measured ultrasound RF signal (4 MHz). The difference in the signal bandwidth between the simulated and measured signal may be attributed to the simplifications used in the simulated signal model.

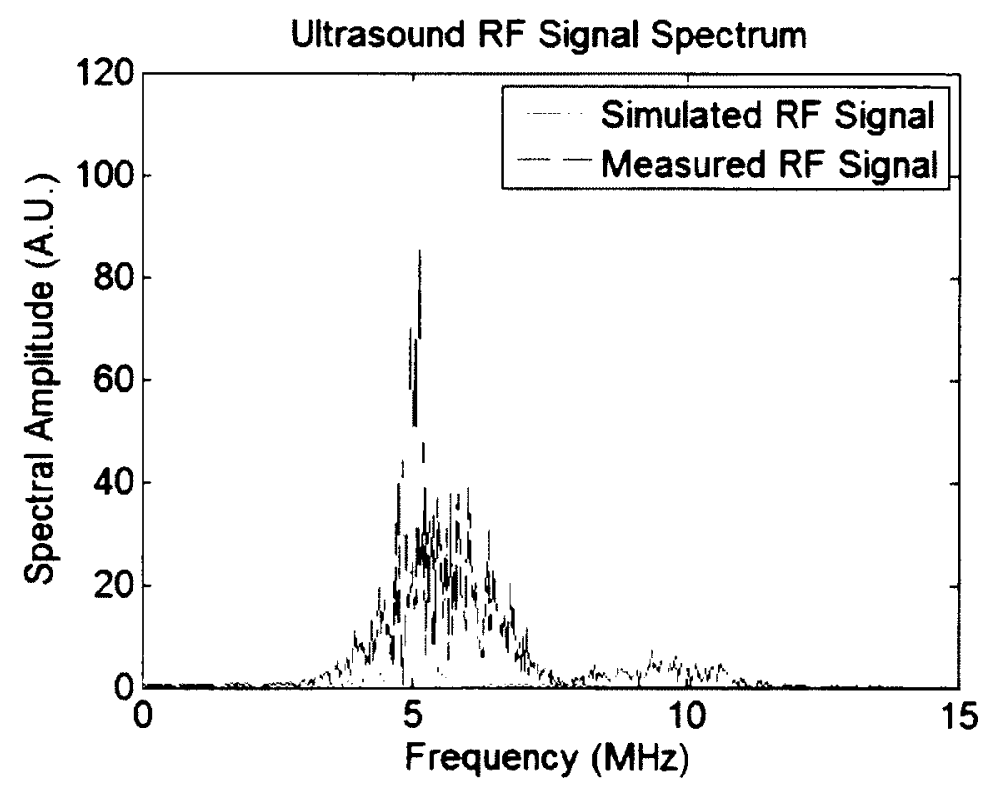

Figure 4.7 Simulated and measured ultrasound RF signal spectrum. The spectral content of the simulated ultrasound RF signal is shown in red, while the spectral content of the measured RF signal is shown in dotted blue. 
Numerical simulation results of the phase RMS error with respect to SNR at different lateral motion distances are shown in Figure 4.8. The plot for lateral motion of $0 \mu \mathrm{m}$ provides a basis for comparison when no lateral motion is present. As the lateral distance increases the observed phase RMS at each relevant SNR level increases. This behaviour matches the intuitive expectation that a larger lateral distance would result in larger phase difference between consecutive frames, and therefore a larger phase RMS error. A lateral motion results not only in a change in the probe measurement area but also changes the relative position of the scatterers along the beam lateral profile.
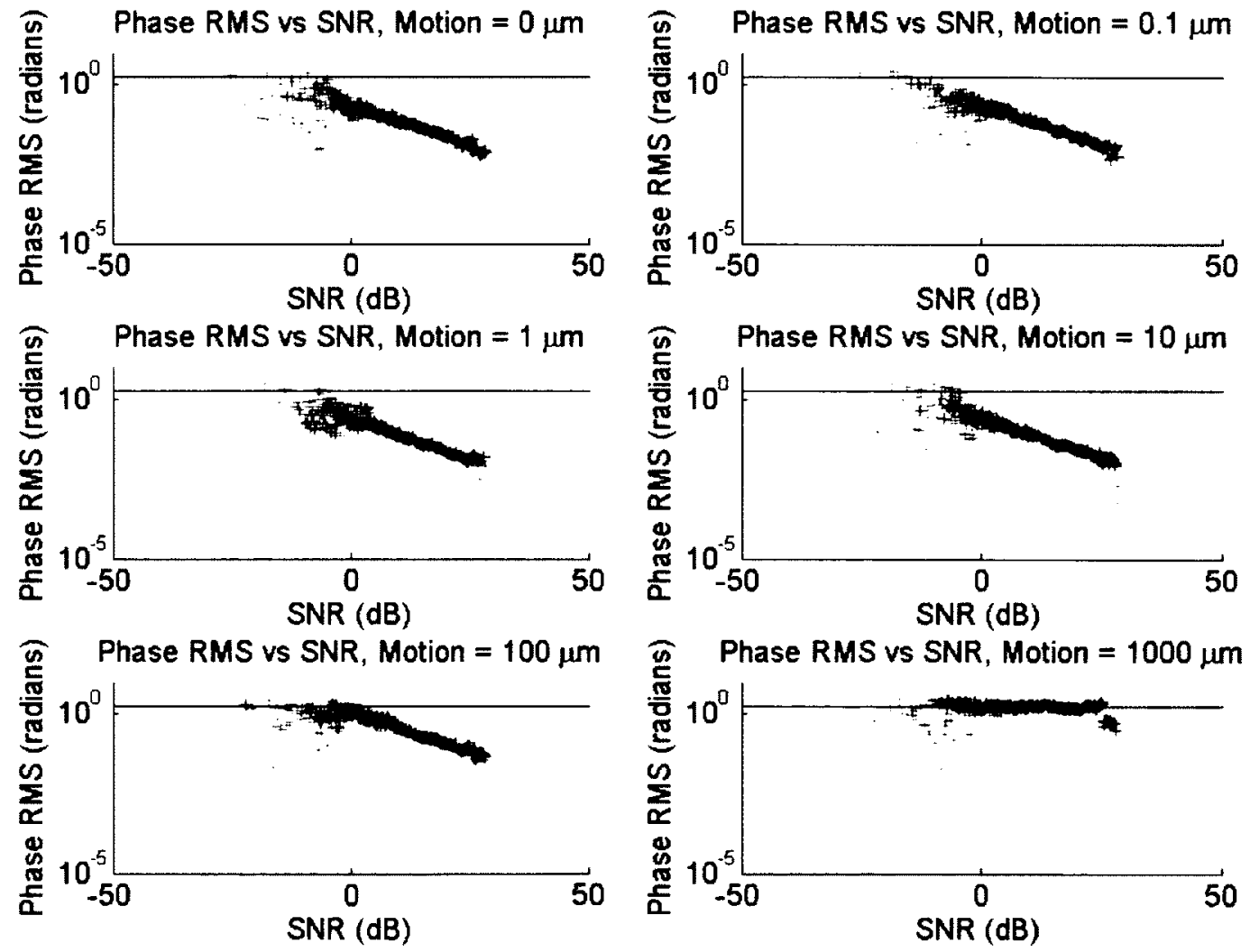

Figure 4.8 Simulation Results. Phase vs SNR data points shown in red. The solid line indicates the value of 1.813 which is the theoretical maximum phase RMS due to phase wrapping. 
The lateral motion causes the probe to measure a different region post-motion compared to pre-motion. This results in a difference in the measured RF signals, and a corresponding difference in the phase values between the two signals. A larger distance exacerbates this problem as the overlap of the pre- and post- motion measurement area decreases. Thus, a larger difference in the RF signals is present and therefore a larger phase difference.

In addition to the change in measurement area there is also a change in the relative position of the scatterers along the ultrasound beam profile. The beam profile is modelled by a Gaussian function as defined by Eq. 4.8. Scatterers location near the probe center produce a stronger reflection echo in comparison to scatterers located near the beam edge. Lateral motion would therefore result in a movement of the scatterers along this beam profile, resulting in a decrease in reflection echo amplitude as the scatterer moves away from the center or vice versa as the echo moves towards the center. The effect of lateral motion is twofold as it results in signal changes from both changes in the measurement area and changes to echo strength along the beam profile.

One issue with measurement dependency on phase information is phase wrapping. Due to phase wrapping, phase differences greater than $\pi$ or $-\pi$ cannot be measured. Due to this it is evident that there is a theoretical maximum to the phase RMS. As the phase difference increases, due to wrapping it is trapped between $\pi$ and $-\pi$. At the maximum possible phase, the phase values would be uniformly distributed between $\pi$ and $-\pi$. Based on this theory, the theoretical maximum phase RMS is calculated from the standard deviation of a uniform distribution between $\pi$ and $-\pi$, and results in a value of 1.813 . This threshold therefore represents the phase RMS that we expect to observe when the estimated phase values are saturated by phase jump errors. 
As the lateral motion distance increases and the phase RMS vs SNR curve shifts upwards the data points approach this theoretical threshold. As the curve reaches the threshold this will result in clipping of the curve as the phase RMS value hits the saturation point. This is most evident for the lateral motion distance of $1000 \mu \mathrm{m}$ where the entire curve is at the threshold. The SNR values with phase RMS error at this threshold are therefore unusable for displacement estimation from the phase due to the phase wrap error. Therefore, for lateral motion distances of $1000 \mu \mathrm{m}$ displacement information cannot be obtained from the ultrasound signal using the phase information. In the plot for $1000 \mu \mathrm{m}$ in Figure 4.8 there are points above the threshold line since the line is an estimate of the phase RMS when the phase information is saturated by phase wrap errors. The theoretical threshold indicates the expected phase RMS when the phase is saturated by phase jump errors. However, the RMS of the phase at certain SNR levels can be higher than this threshold. If an artificial large displacement is observed due to poor SNR then the majority of the phase values at this SNR are close to $\pi$ or $-\pi$. The RMS of a group of phase values closer to $\pi$ or $-\pi$ will be larger than then theoretical threshold. Therefore while phase RMS values can exist above this threshold, the SNR levels that result in the aforementioned phase RMS values cannot be used in displacement estimation. The lateral motion distance of $1000 \mu \mathrm{m}$ at the clinical ultrasound imaging $\mathrm{M}$-mode frame rate of $980 \mathrm{~Hz}$ corresponds to a lateral motion velocity of $0.98 \mathrm{~m} / \mathrm{s}$. This lateral motion distance and velocity represents an extreme worst case performance. Therefore in most measurement setups it is likely that ultrasound RF signals with large SNR can still be used in phase sensitive displacement estimation. 


\section{8}

\section{Phase RMS vs Lateral Motion}

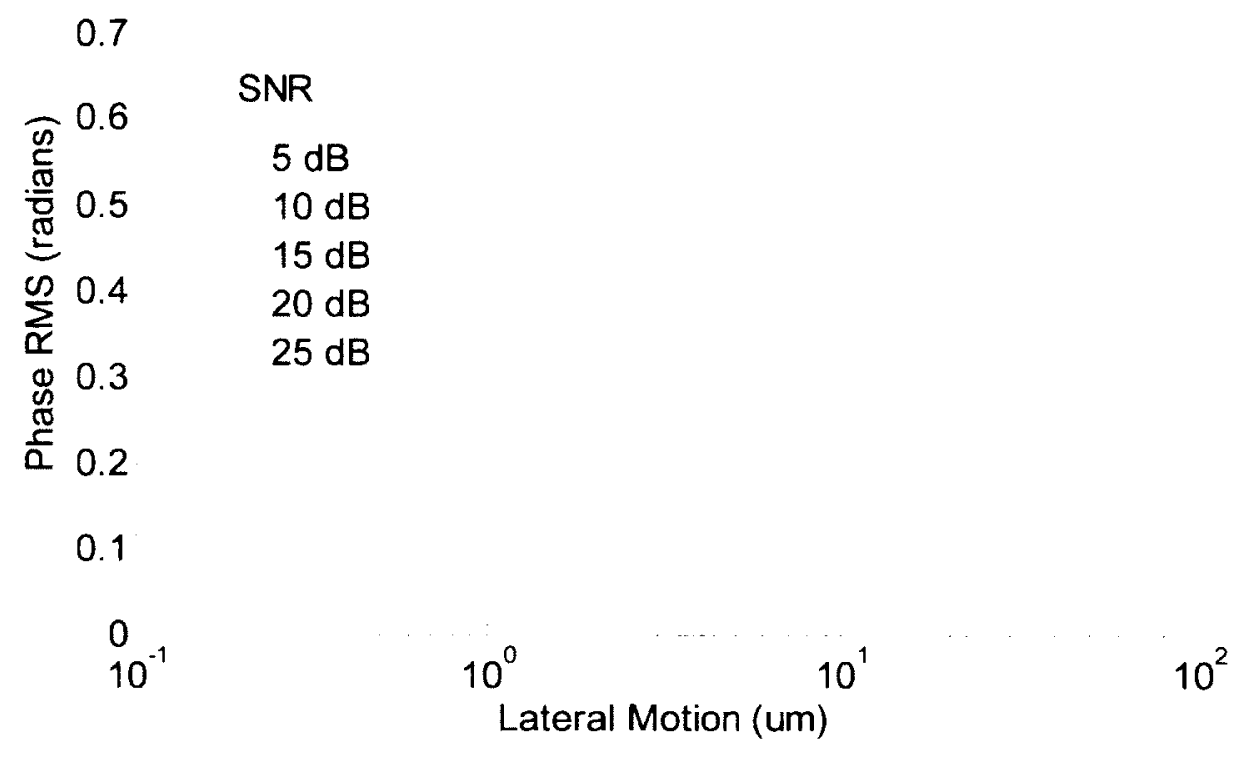

Figure 4.9 Phase RMS vs Lateral Motion for Different SNR Levels

While the relationship between phase RMS and SNR is one important part of our analysis, the influence of the lateral motion distance on the phase RMS is also crucial to determine. This information could assist in determination of the level of acceptable lateral motion, and provides a clear picture of the impact of lateral motion.

In Figure 4.9 we can see the result of the phase RMS error for different distances of lateral motion at select SNR values. The plot shown in Figure 4.9 is derived from Figure 4.8 by collating phase values at depths corresponding to the desired $\mathrm{SNR}$ value $\pm 0.5 \mathrm{~dB}$ at each lateral motion distance. The collected phase information at each SNR value is then used to calculate the phase RMS at the specific SNR value at each lateral motion distance.

From Figure 4.9, the phase RMS values appear to be relatively constant below $10 \mu \mathrm{m}$ of lateral motion distance at each SNR value used in this simulation, while they increase with the lateral motion above $10 \mu \mathrm{m}$. A lateral distance of $10 \mu \mathrm{m}$ corresponds to the motion velocity of 
$10 \mathrm{~mm} / \mathrm{sec}$ when the frame rate is $1000 \mathrm{~Hz}$. As the SNR values decreases, the phase RMS value increases for a given lateral motion.

\subsection{Phantom Simulation Experiment}

A phantom provides an essential simulation environment that bridges the gap between numerical simulations and in vivo experiments. The numerical simulation provides an initial idea of the effect of lateral motion in a controlled environment. However, the numerical simulation only considers a small number of error sources in the ultrasound measurement. Phantom simulation provides the capability to investigate the effect of lateral motion in a more realistic environment.

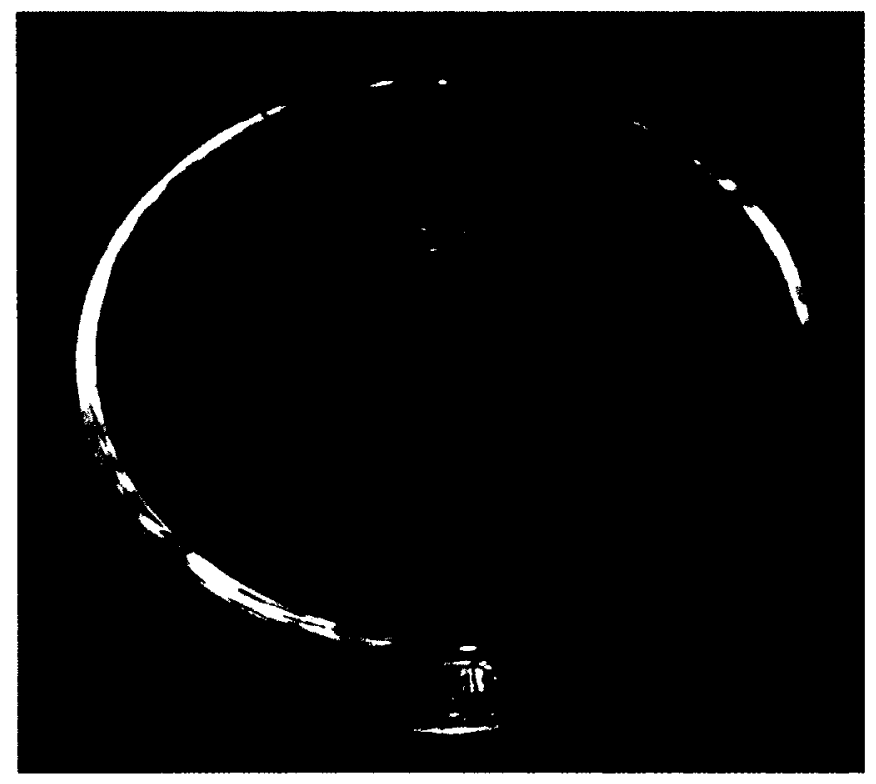

Figure 4.10 3-w\% Agar Phantom with 3-w\% graphite powder.

The tissue-mimicking phantom used in this experiment was a $3-w \%$ agar phantom with a thickness of $2 \mathrm{~cm}$ and a diameter of $12.6 \mathrm{~cm}$. In addition to the agar, $3-\mathrm{w} \%$ graphite particles (nominal diameter: $50 \mu \mathrm{m}$ ) were added as ultrasound scatterers. The constructed phantom can be seen in Figure 4.10. 


\subsubsection{Experimental Procedure}

The purpose of this experiment is to investigate the effect of probe lateral motion on the phase estimation accuracy of actual ultrasonic RF signals. In order to collect accurate results it is required to ensure that the phantom state is independent of any probe motion. To achieve this condition, the phantom is submerged in a water bath while the ultrasound probe performs measurements near the water surface so that there is a water layer between the phantom and probing face. When performing ultrasound measurements a coupling agent is required to avoid any air pockets between the probe-measurement surface and a subject. The probe-air interface has a large reflection coefficient and results in a near total reflection of the transmitted signal. A coupling agent is therefore used to fill any gaps between the ultrasound probe and measurement surface. Typically in clinical settings, an ultrasound coupling gel is used; however water is also an excellent coupling agent. Therefore, the water in the water bath provides a coupling medium between the probe and phantom to allow ultrasound measurements at a distance from the probe.

Due to the lack of direct contact between the ultrasound probe and phantom surface, the probe lateral motion will not induce any phantom movement. 


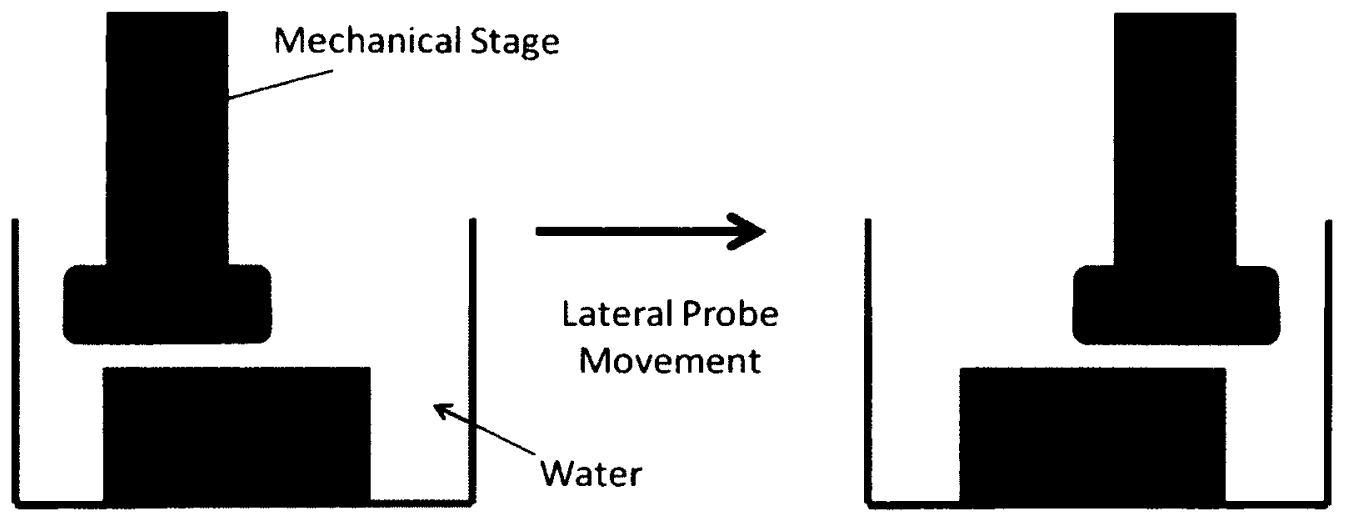

Figure 4.11 Model of phantom experiment setup. Phantom is placed in water bath while mechanical stage moves probe laterally at a constant velocity.

The model of the experiment setup is shown in Figure 4.11. The phantom is submerged in a water bath and the ultrasound probe is secured in a metal holder attached to a rigid aluminum beam. This aluminum beam is secured to the mechanical stage and is used to avoid undesirable probe sway or vibration in the experiment. The probe is lowered until the probe surface is underwater. A picture of the experiment setup is shown in Figure 4.12. 


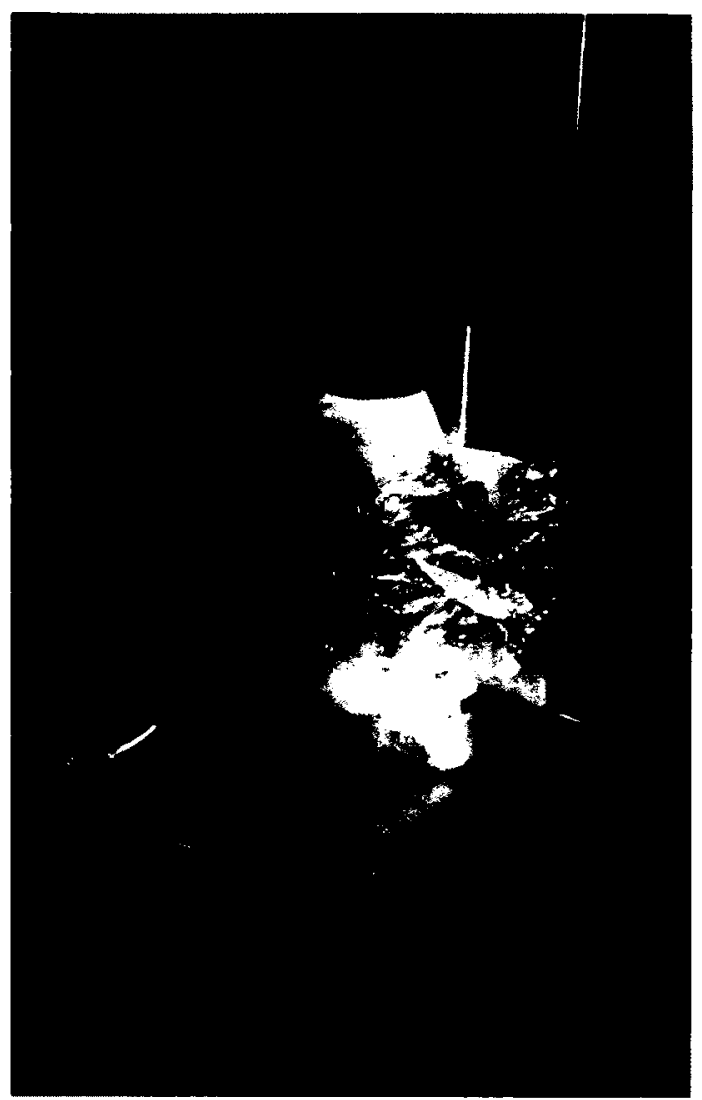

Figure 4.12 Phantom Motion Noise Experimental Setup. The phantom is submerged in a water bath. The probe is secured to a metal holder and lowered until measurement surface is submerged.

The mechanical stage is used to move the probe at a constant rate of lateral motion. The movement velocities are selected to achieve approximately $0 \mu \mathrm{m}, 0.1 \mu \mathrm{m}, 1 \mu \mathrm{m}, 10 \mu \mathrm{m}$, and 100 $\mu \mathrm{m}$ lateral movement distances between two consecutive measurement frames. For the $0 \mu \mathrm{m}$ lateral movement case, no motion is applied by the mechanical stage.

Each measurement records $5.4 \mathrm{~s}$ of experimental data. Every measurement begins with no motion to collect ultrasonic RF signals for $1 \mathrm{~s}$ to obtain the inherent noise of the setup. This is followed by probe movement at the desired velocity for $3 \mathrm{~s}$, and no motion for final $1 \mathrm{~s}$. Four measurements are conducted for each lateral motion distance to ensure reproducibility of results.

From the measurements, the $1 \mathrm{~s}$ of data at the start of each measurement is used to estimate the noise standard deviation at each depth. These estimates are used to calculate the 
SNR of acquired ultrasound RF signals at each depth. The acquired ultrasound RF signals with lateral motion are extracted from the $3 \mathrm{~s}$ of motion period where the probe is moving at a constant velocity. Quadrature demodulation is applied to extract the phase value of the acquired ultrasonic RF signal. From the phase value and SNR, the phase RMS error for specified SNR levels can be calculated based on the steps discussed in Section 4.3.3.

\subsubsection{Phantom Experiment Results}

The phase RMS error and SNR results obtained from the phantom experiment are shown in Figure 4.13. The plots show the phase RMS error and SNR values at different lateral motion distances along with the numerical simulation results for comparison purposes. No experimental data was available for the $1000 \mu \mathrm{m}$ scenario due to hardware limitations of the speed of the translation stage.

The results shown in Figure 4.13 validate the models used in the numerical simulation. The numerical simulation and phantom experiment results demonstrate a good agreement over the relevant SNR ranges.

The two primary error sources of phase estimation in the numerical model are the AWGN noise and the lateral motion. However, additional noise sources are likely present in the actual experiment setup. Potential noise sources include environmental noise that causes minor vibrations in the experiment apparatus. 

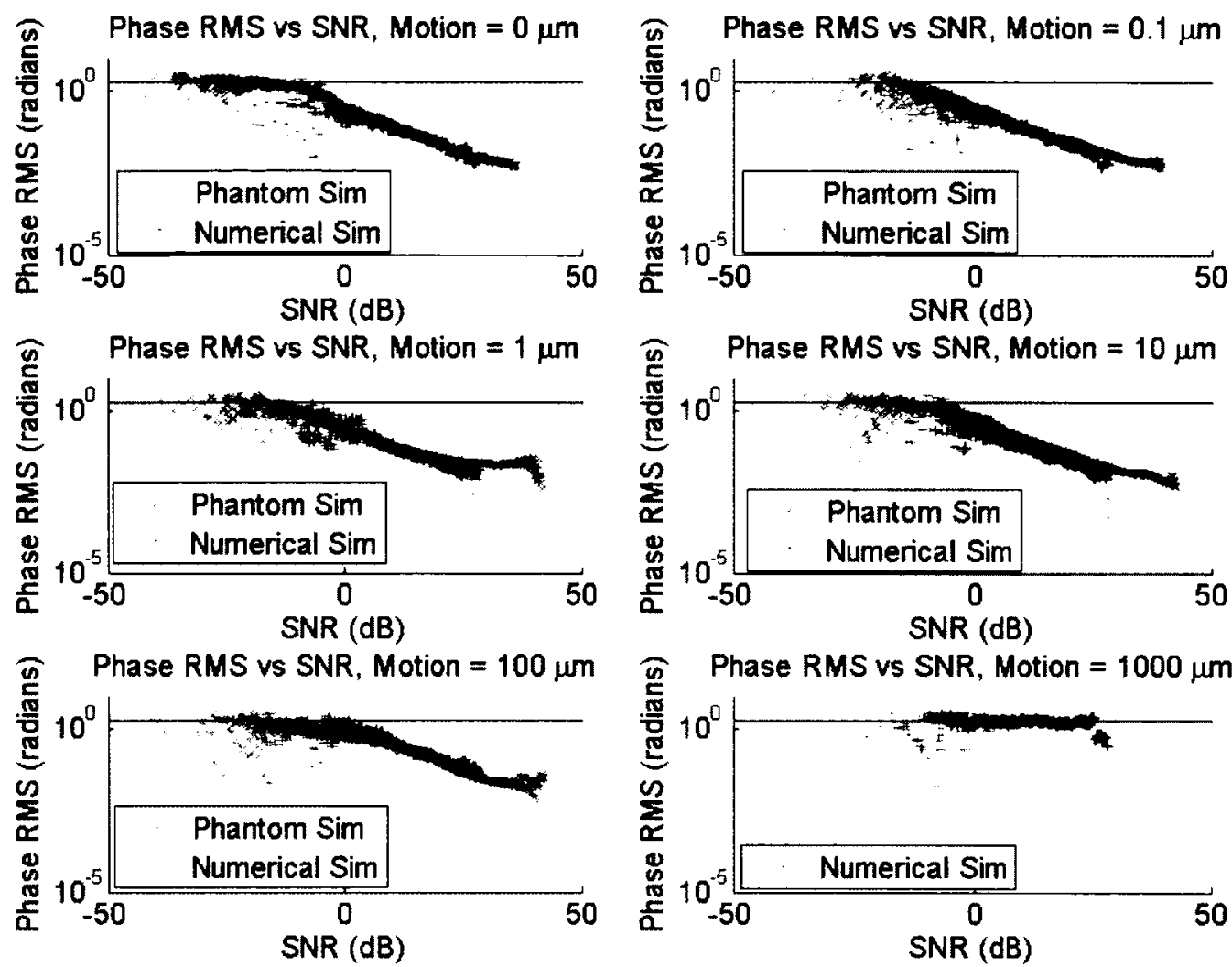

Figure 4.13 Phase RMS and SNR plot generated from phantom experiment and numerical simulation. Results from the phantom experiment are in shown in red while the results from the simulation are shown in blue. The horizontal black line shows the theoretical maximum SNR threshold.

In addition to the unaccounted noise sources are the estimation used in the phantom experiment for SNR calculations. The random noise is parameterized by its standard deviation. In the phantom experiment, the first $1 \mathrm{~s}$ of the measurement period is used to estimate the standard deviation of the noise. The noise standard deviations in the SNR calculation for the phantom experiment are therefore estimates of the random noise present in the signal. However, for the numerical simulation the exact value of the noise standard deviation is known. The estimated standard deviation varies around the actual value; therefore standard deviations both 
larger and smaller than the actual value will be obtained. From Eq. 4.2 it can be deduced that the varying standard deviation will result in a larger range of observed SNR.

The larger range of SNR observed in the phantom experiment provide the ability to observe the effect of the phase RMS error threshold. The black lines in Figure 4.13 indicate the maximum theoretical threshold value (1.813) for the phase RMS error when the phase information is saturated by phase wrap errors. While the majority of the numerical simulation results do not hit the threshold, due to the larger range of SNR values in the phantom measurements the effect of the threshold is observed on lateral measurement distances $1 \mu \mathrm{m}$ and greater.

The phase RMS error vs SNR for these points appear to show a decrease in phase RMS error with SNR. In other words, as the phase SNR decreases the phase RMS is increasing. However, this pattern halts when the phase RMS reaches the theoretical threshold and the phase RMS levels out. As discussed in Section 4.3.5 the SNR values in this saturation section are unusable for displacement estimation. It is evident from visual observation that the SNR value where the saturation period begins increases with the lateral motion distance. Therefore, as the lateral motion distance increases the usable segments of the received ultrasound reflection echo signal decreases.

\subsection{Moving Average Filter for Random Noise Reduction}

In addition to lateral motion causing displacement estimate error by the change in measurement area, another source of error to consider is random noise appearing in the ultrasonic RF signal as discussed in Sections 4.3 and 4.4. Random noise results in variations in the ultrasound RF signal that are unassociated with muscle movement and result in errors in the 
displacement estimate. To reduce the errors that result from random noise we propose the use of a moving average filter to reduce the random noise.

The moving average filter performs an ensemble averaging on ultrasound RF signal acquired from consecutive M-mode frames. The moving average takes subsets of five consecutive M-mode frames and computes the average of the five ultrasound RF signal from each frame. The application of the moving average filter results in a reduction of the random noise in ultrasound RF signal. However, the averaging of several $\mathrm{M}$-mode frames results in a blurring and reduction in the accuracy of the temporal resolution of the displacement measurement.

The results of the application of a moving average filter, where five consecutive RF signals are averaged are shown in Figure 4.14 with the original data for comparison purposes. The groupings of the calculated phase RMS value use the SNR values without the moving average filter. From Figure 4.14 the decrease in phase RMS at each SNR level due to the moving average filter can be discerned.

Under the conditions employed in these experiments, the moving average filter resulted in a decrease in the phase RMS across all SNR values at lateral motion distances of $1 \mu \mathrm{m}$ and less. For $10 \mu \mathrm{m}$ and $100 \mu \mathrm{m}$ lateral motion distances, the moving average filter decreased the phase error RMS for small SNR values, but at larger SNR the moving average filter does not appear to have improvement. 

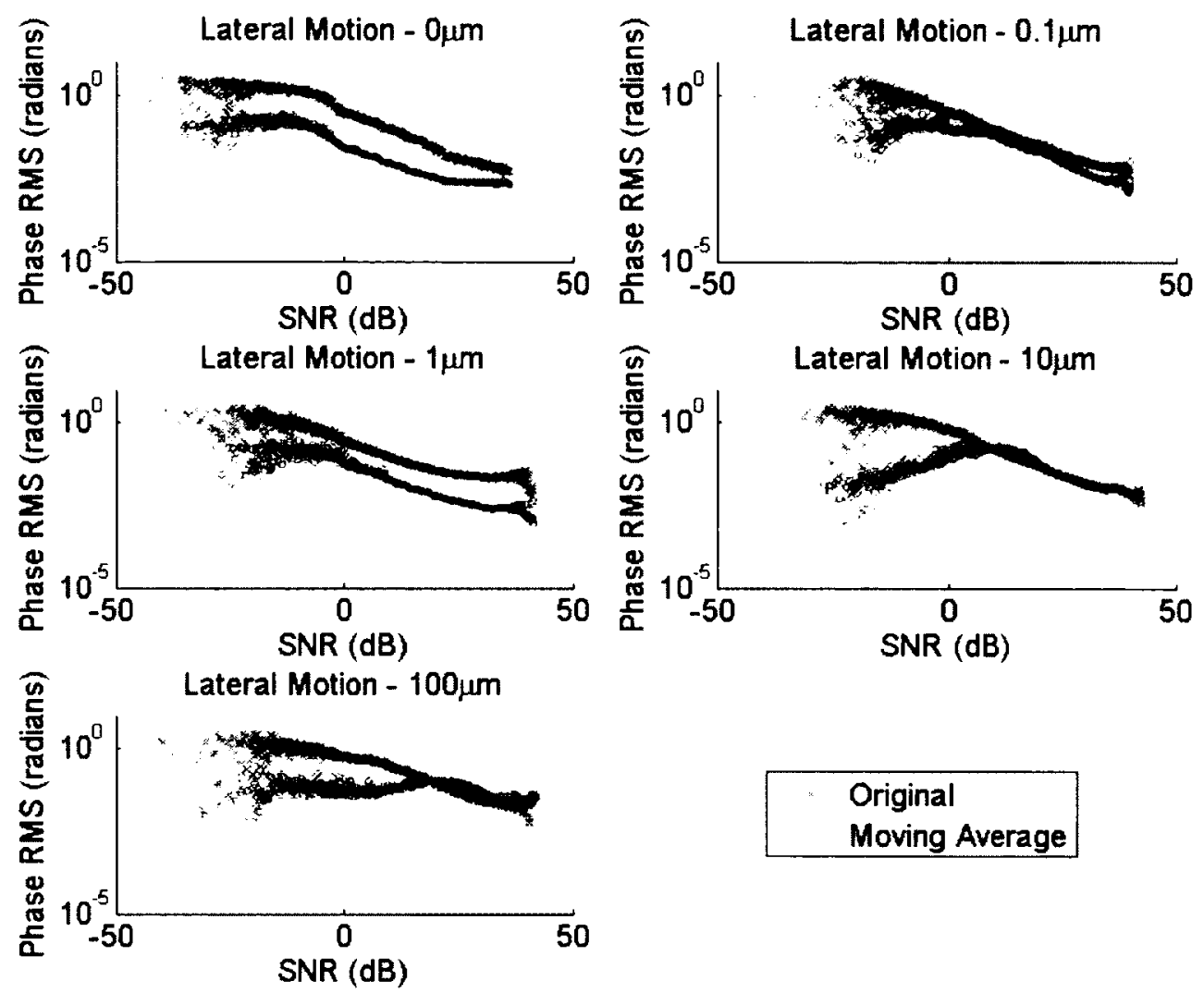

Original

Moving Average

Figure 4.14 Phantom Experimental Results with and without Moving Average Filter of 5 samples. The original data are in blue, while the moving average results are given in red.

Based on the numerical simulation results shown in Figure 4.9 , lateral motion distances less than $10 \mu \mathrm{m}$ does not show any change in the phase RMS errors. In addition, the RMS error decreases with the greater SNR. Therefore, the dominant cause of phase errors observed in the measurements below $10 \mu \mathrm{m}$ is due to the random noise. The random noise reduction by the moving average filter results in a reduction in the phase error across all SNR levels for the lateral motion distances of $1 \mu \mathrm{m}$ and below in the phantom simulation experiment conditions employed in this study.

As the lateral motion distance increases (in the cases of $10 \mu \mathrm{m}$ and $100 \mu \mathrm{m}$ in Figure 4.14), the lateral probe motion becomes the predominant error source. However, at the SNR 
levels below $10 \mathrm{~dB}$ for the motion of $10 \mu \mathrm{m}$ and $20 \mathrm{~dB}$ for $100 \mu \mathrm{m}$ as seen in Figure 4.14 , the phase errors are likely still dominated by random noise and, therefore, error reduction is observed with the moving average filter. However, as the SNR increases, the lateral motion probably becomes the dominant error source and the phase errors are not reduced by the moving averaging filter. 


\section{Vertical Displacement Measurements}

In Chapter 4, the effect of lateral probe motion on ultrasound measurement of internal displacement in static objects was examined. In this chapter the effect of lateral motion of ultrasonic probe and/or internal tissue is investigated in ultrasound measurement of vertical tissue displacement. Initial investigations are performed by phantom simulation experiments where lateral compression is applied to the phantom to simulate muscle contraction. The effect of the moving average filter to remove random noise and improve measurement accuracy is also examined. Afterwards, in vivo experiments are performed at a forearm of a human subject to examine the lateral motion effect on internal tissue displacement measurements.

\subsection{Phantom Simulation Experiments}

In order to simulate tissue displacement due to skeletal muscle contraction, lateral compression is applied to a phantom. A $3-\mathrm{W} \%$ agar phantom containing fine carbon particles as ultrasound scatterers is used to mimic the skeletal muscle tissue. Lateral compression applied to the phantom introduces a vertical displacement parallel to the ultrasound propagation direction.

\subsubsection{Experimental Setup and Procedure}

The experiment setup is the same as the one used in the Chapter 4, shown in Figure 4.11. The $2.5 \mathrm{~cm}$ thick circular phantom with radius $8 \mathrm{~cm}$ is submerged in a water bath. The phantom used in this experiment is different from the phantom used in Chapter 4; however both phantoms were constructed based on the same methodology. The ultrasonic probe is secured to a metal plate attached to the mechanical stage and lowered until the probing surface is under water. 
Lateral compression is applied to the phantom to simulate muscle movement during skeletal muscle contraction. Two different methods are used to apply the compression: Plexiglas plate and hand compressions. Phantom lateral compression from the Plexiglas plates is performed by first placing the phantom between two Plexiglas plates. One plate is then moved towards the other to compress the phantom by $3 \mathrm{~mm}$. For hand compression the phantom is held in one hand with the fingers gripping the phantom sides. The phantom is then laterally compressed by squeezing the fingers together. Both Plexiglas or hand compression are applied for a period of $2 \mathrm{~s}$ and then released. This compression-release cycle is repeated twice in each measurement. The Plexiglas plate allows the phantom to be compressed in a more uniform manner as force is applied over an entire phantom side surface. Conversely, the hand compression is less uniform and more localized. The clinical ultrasound imaging system is used to perform M-mode measurements for $5.5 \mathrm{~s}$. Each measurement period begins with a reference period of $1 \mathrm{~s}$ with no compression for noise estimation. This is followed by $3 \mathrm{~s}$ of compression where the phantom is laterally compressed and released twice based on the procedure described above.

\subsubsection{Experimental Results}

The vertical displacement that occurred within the phantom can be estimated from ultrasonic RF signals acquired in M-mode measurement using the quadrature detection technique as described in Chapter 2. The quadrature detection technique examines the change in phase of ultrasonic RF signals between consecutive measurement frames to estimate the movement of scatterers at a particular depth. From the phase changes in the acquired ultrasound RF signals we are then able to estimate the vertical displacement that occurs throughout the phantom along the 
ultrasound beam direction. The maximum measurement depth is $35 \mathrm{~mm}$ with the ultrasound imaging system used in this thesis research.

An example of the acquired ultrasound RF signal with a phantom is shown in Figure 5.1. From the water/phantom interface at a depth of $2 \mathrm{~mm}$, ultrasonic signals start to be observed. The phantom is submerged in a water bath, thus no echoes appear while the transmitted ultrasound beam travels through the water. As the transmitted wave travels into the phantom echoes appear as it encounters the ultrasound scatterers randomly distributed within the phantom. The large reflection echo occurs once the transmitted ultrasound beam reaches the phantom bottom and encounters the Plexiglas container surface. The large acoustic impedance difference between the phantom and Plexiglas results in a large reflection echo at a depth of $31 \mathrm{~mm}$ (phantom thickness of $25 \mathrm{~mm}$ plus water layer thickness of $3 \mathrm{~mm}$ ) as highlighted in Figure 5.1 .

The displacement estimation obtained through quadrature detection from the phantom compression experiment using Plexiglas plates is shown in Figure 5.2. This 3-dimensional plot shows the displacement that occurs within the entire depth within the ultrasonic beam over the compression period. The lateral compression of the phantom results in a vertical displacement within the phantom. 


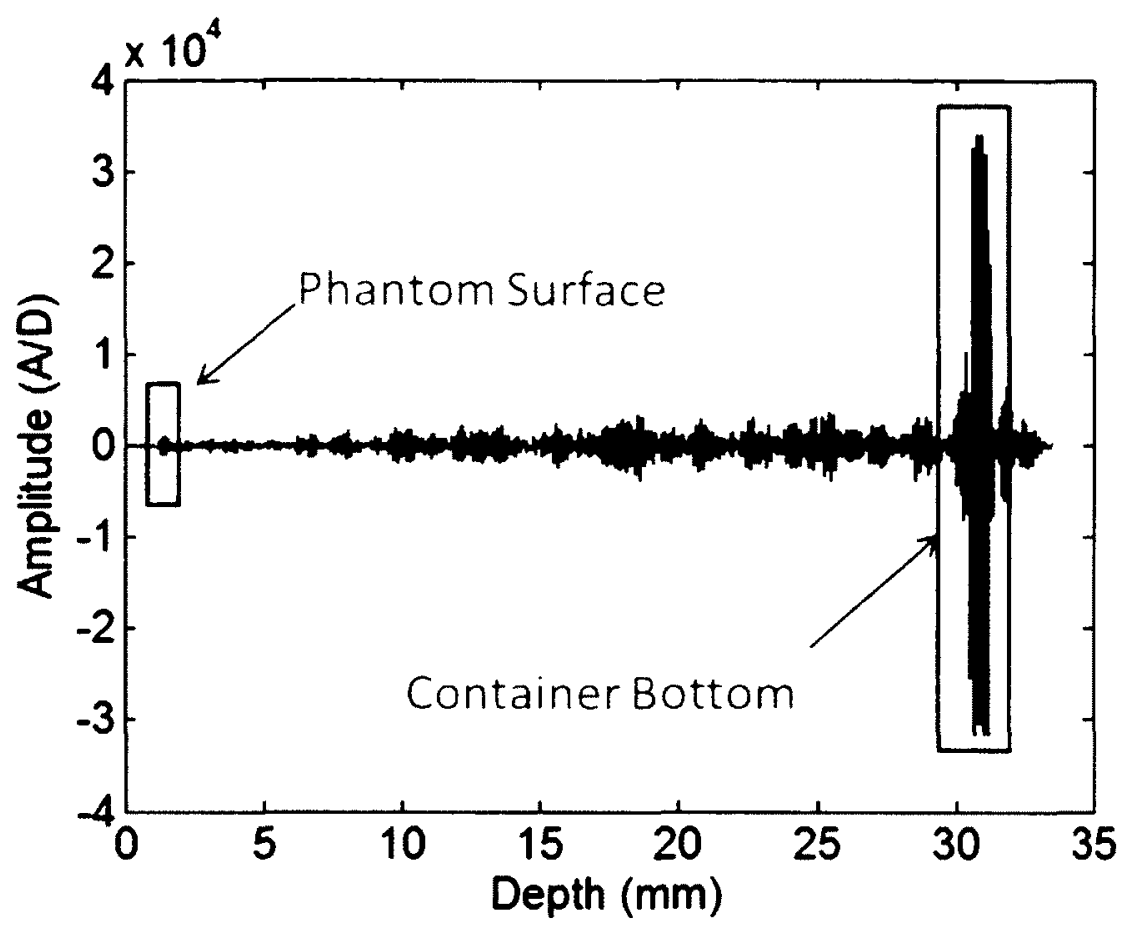

Figure 5.1 UItrasound RF signal from phantom measurement

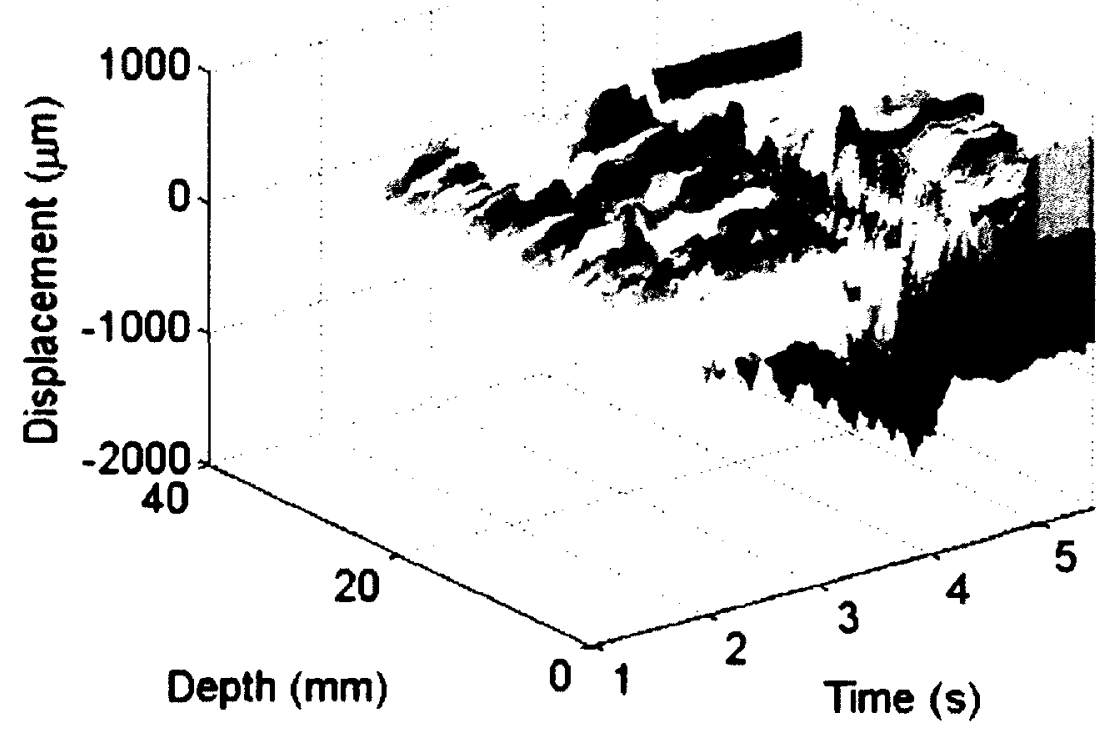

Figure 5.2 Vertical displacement observed in phantom by Plexiglas compression. 
Due to technical constraints it was not possible to directly measure the internal vertical displacement within the phantom during compression. Therefore, another method using a different approach is used to calculate the internal displacement for comparison purposes. In order to verify the accuracy of the quadrature detection technique the obtained results are compared to the cross-correlation method. A close match between the results from quadrature detection (phase sensitive method) and cross-correlation (template matching method) lends credence that the results from quadrature detection are accurate.

The displacement estimates of the top surface of the phantom from the two methods are shown in Figure 5.3. In this figure the vertical distance (distance in direction parallel to the transmitted ultrasonic beam) from the ultrasound probe to the phantom surface is plotted. The cross-correlation uses a template width $280 \mu \mathrm{m}$ (approximately one wavelength of ultrasound) and searches over an area of $370 \mu \mathrm{m}$ centered at the phantom surface. The template size was selected as one wavelength to ensure that one period of ultrasonic RF signal was captured. The search area size was selected to encompass the potential movement range of the surface between consecutive frames while minimizing the search size to reduce computation time. Similar to the quadrature detection technique, cross-correlation is used to estimate the vertical displacement that occurs between two consecutive frames. In order to increase the accuracy of crosscorrelation estimate, an interpolation filter is applied to upsample the acquired ultrasound RF signal from $33.3 \mathrm{MHz}$ to $333 \mathrm{MHz}$. This increased the temporal resolution of cross-correlation from $23.1 \mu \mathrm{m}$ to $2.31 \mu \mathrm{m}$.

From comparison of the displacement estimates from the two techniques as seen in Figure 5.3 the quadrature detection and cross-correlation show similar displacement estimates. In the periods with small or slow movement between $0 \mathrm{~s}$ and $3.85 \mathrm{~s}$, the techniques essentially 
produce almost identical displacements. The divergence between the two techniques occurs when the rate of displacement increases at around $4 \mathrm{~s}$ where a large negative displacement has occurred. After the divergence between the displacements estimates from the quadrature demodulation and cross-correlation techniques, the shape of the displacement profiles appear similar but there is an offset between the distance plots due to the resulting divergence after 4.5

S.

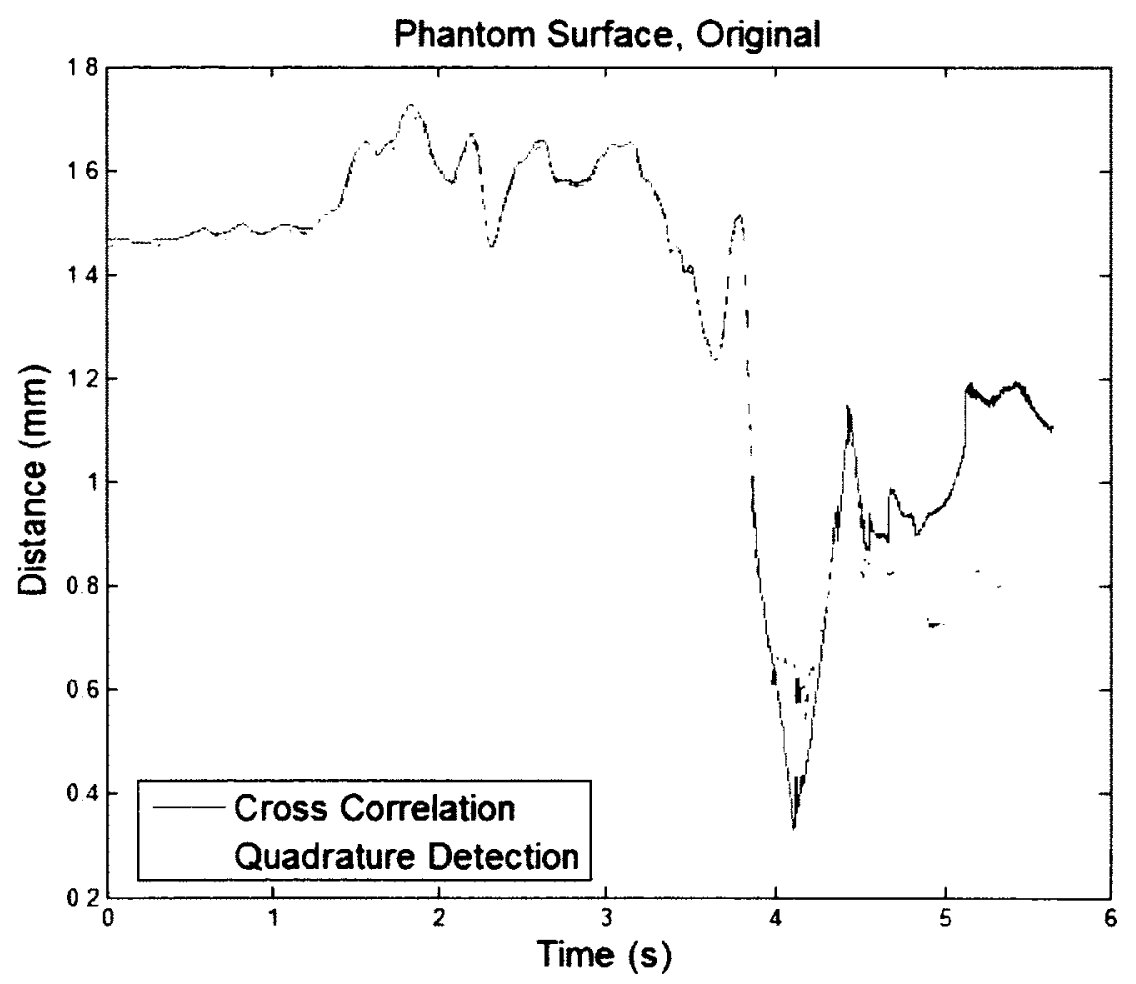

Figure 5.3 Distance of phantom surface distance from ultrasound probe during Plexiglas compression. Cross-correlation estimate is shown in solid blue line, and quadrature demodulation is shown in red dashed line.

The divergence between the two distance plots may be due to the difference between the parameters estimated by the quadrature detection and cross-correlation techniques. Quadrature detection estimates the displacement at a particular depth. To obtain the surface displacement, the displacement that occurs at the original surface depth is calculated. To convert the distance 
measure shown in Figure 5.3 the distance from the ultrasound probe to the original depth is added to the quadrature detection displacement estimate. Therefore, quadrature detection obtains a displacement estimate at the original surface depth. On the other hand, cross-correlation estimates the displacement based on shifts in template location within the ultrasound signal. Cross-correlation therefore directly estimates the change in distance from the ultrasound probe to phantom surface. This difference in definition may be one reason for the difference in the two distance plots in Figure 5.3.

For small displacement the phantom surface is still close to the original depth. The estimation of displacement at the original depth from quadrature detection is therefore close to the actual displacement of phantom surface obtained from cross-correlation. As the displacement increases the location of the phantom surface moves away from its original location. Therefore, the displacement estimate from the quadrature detection is not the actual surface displacement estimated from the cross-correlation technique but a displacement at the original surface depth.

However, Figure 5.3 shows that the estimated displacement from quadrature detection is close to the estimated movement obtained by cross-correlation. The cross-correlation technique utilizes a subset of the signal to generate the template with a larger template resulting in greater displacement estimation accuracy. However, a larger template also limits the spatial resolution of the displacement estimation. Compared to the cross-correlation method, the quadrature detection techniques provide the capability to estimate the displacement over a range of depth with a higher spatial resolution.

As mentioned in Chapter 2, the quadrature detection technique offers a better performance in tracking of low amplitude speckle echoes compared to the cross-correlation 
method. The quadrature detection technique is able to measure the displacement at any depth. This allows quadrature detection to exhibit similar performance in speckle echoes compared to medium boundaries. Therefore, the quadrature detection technique provides better capability to estimate the displacement within a medium compared to the cross-correlation method.

From the displacement results in Figure 5.3 it appears that the surface distances at the beginning $(0 \mathrm{~s})$ and the end $(5.5 \mathrm{~s})$ are different. No compression is applied to the phantom at the start and end of the measurement period. Therefore, the vertical distance of the phantom surface from the ultrasound probe at $0 \mathrm{~s}$ of measurement should be similar to the vertical distance at 5.5 s. However, from the results shown in Figure 5.3 there appears to be approximately $0.5 \mathrm{~mm}$ and $0.7 \mathrm{~mm}$ change in vertical distance from the cross-correlation and quadrature detection displacement estimates, respectively. As there is no compression applied at either measurement time, the difference in vertical distance is likely the result of lateral movement of the phantom due to a slide between the Plexiglas and phantom so that the phantom measurement area changed during the compression.

To verify the observations obtained from the estimated displacements from the phantom experiment with Plexiglas plate compression, quadrature detection is applied to estimate the displacement within the phantom when manual hand compression is applied instead. Hand compression provides a less uniform compression force when compared to the Plexiglas compression. However, hand compression of the phantom is also more stable (firmer grip of the phantom) and results in less lateral motion compared to the Plexiglas compression. It is difficult to quantify the difference in lateral motion between the Plexiglas and hand compression. However, visual observation of the phantom during Plexiglas compression revealed sliding of the phantom along the Plexiglas plate during compression. This behaviour was not observed in 
the hand compression. The hand compression therefore provides for comparison purposes a set of results that has less lateral motion displacement estimation errors but a less even compression.

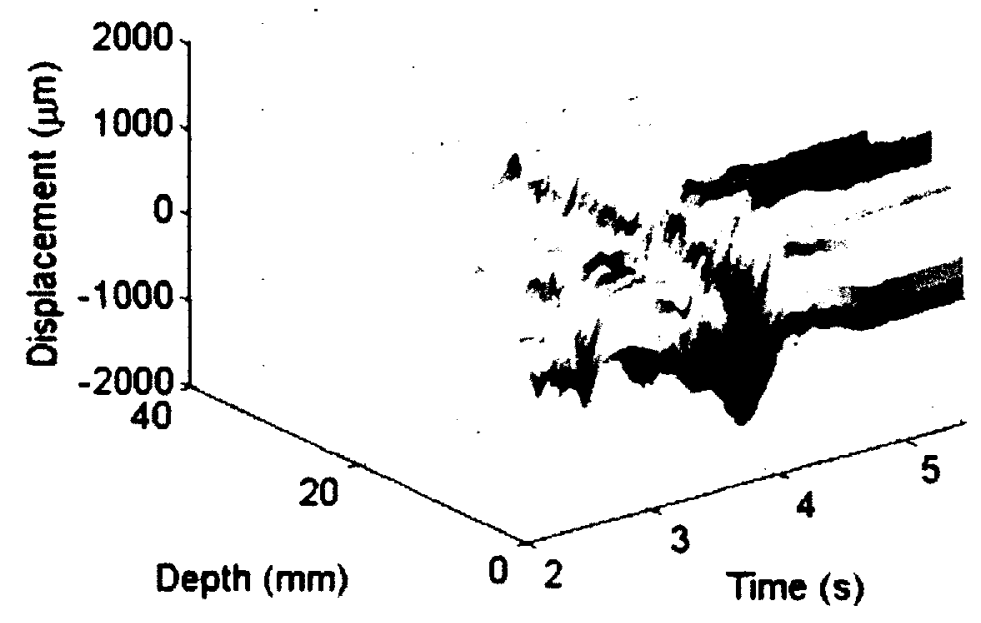

Figure 5.4 Phantom vertical displacement from hand compression using a quadrature detection.

The results of the displacement estimated from quadrature detection are shown in Figure 5.4 , and provides the displacement across the entire depth over the measurement period. 


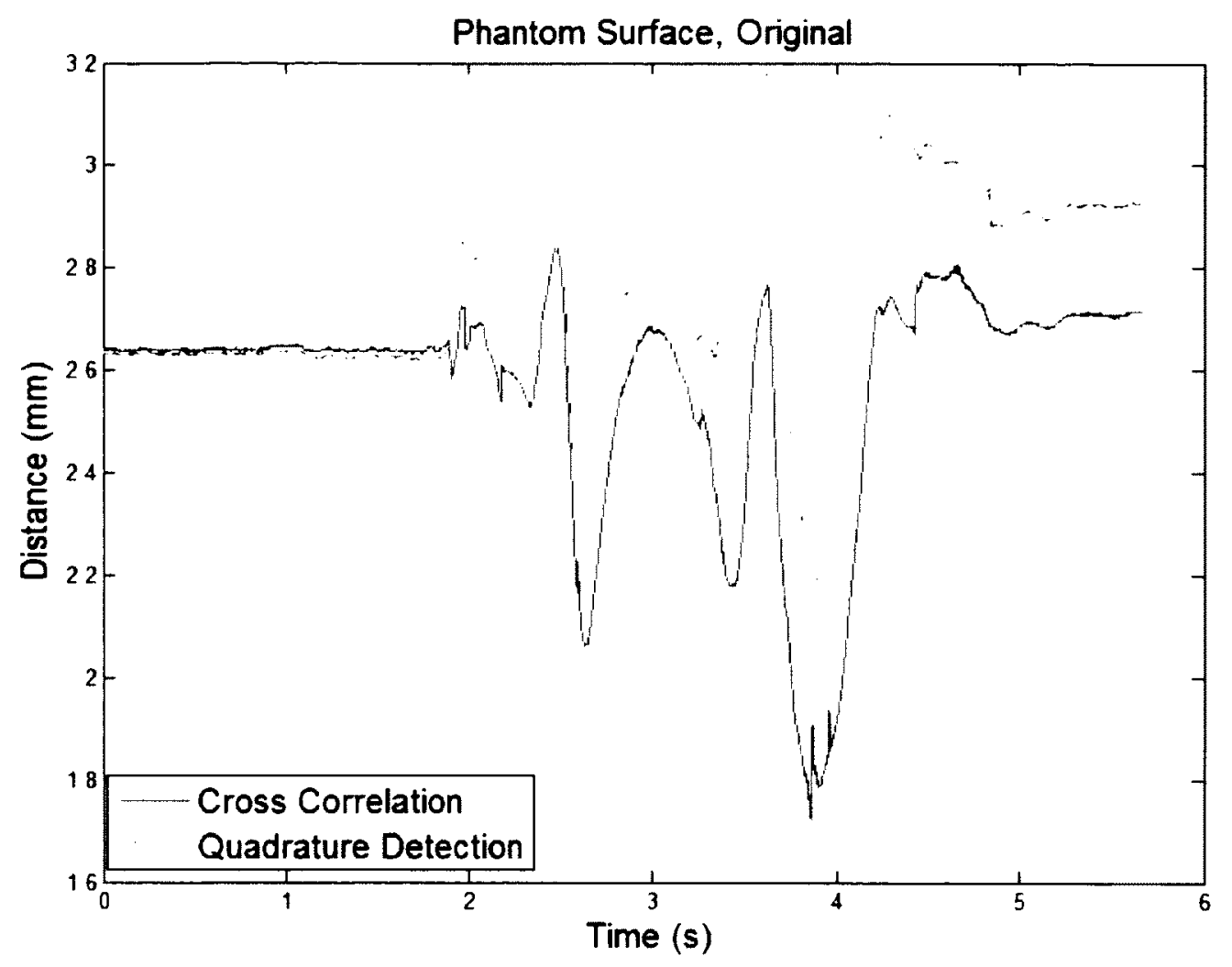

Figure 5.5 Distance of phantom surface distance from ultrasound probe during hand compression. Cross-correlation estimate is shown in solid blue line, and quadrature demodulation is shown in red dashed line.

The comparison of the surface displacements obtained by the quadrature detection and cross correlation is shown in Figure 5.5. Similar to the results from the Plexiglas compression shown in Figure 5.3, the vertical distances from the ultrasound probe obtained from quadrature detection and cross-correlation show similar tendency with an offset between the values from the two methods. The hand compression of the phantom was expected to result in less motion displacement errors. The vertical distances at $0 \mathrm{~s}$ and $5.5 \mathrm{~s}$ of the measurement period are both estimated when no compression is applied to the phantom. The vertical distance obtained from cross-correlation at $0 \mathrm{~s}$ and $5.5 \mathrm{~s}$ of the measurement period are within $0.05 \mathrm{~mm}$ which is much smaller compared to the Plexiglas compression $(0.4 \mathrm{~mm})$. From the cross-correlation results the 
vertical distance at the start and end of the measurement period are similar and it is likely that little global motion was applied to the phantom. Conversely, however, from the quadrature detection technique, the vertical displacement at $0 \mathrm{~s}$ and $5.5 \mathrm{~s}$ are $2.65 \mathrm{~mm}$ and $2.9 \mathrm{~mm}$ respectively. The deviation between the quadrature detection and cross-correlation displacements can be partially explained by the difference in calculated parameter as explained above. The difference in vertical distance obtained from quadrature detection at the start and end of the measurement is likely due to an error in the quadrature detection calculations. The possible cause of this error is due to phase jump error that occurs when the instantaneous displacement (or displacement between two consecutive frames) exceeds half of the ultrasound wavelength.

Phase jump errors also occur when the difference in phase between two consecutive frames exceeds $2 \pi$ due to the random noises in the RF signals when the SNR of the RF signal is poor, resulting in phase wrapping. At the M-mode operating frame rate of $980 \mathrm{~Hz}$ and ultrasound frequency of $5 \mathrm{MHz}$, this instantaneous displacement distance corresponds to an instantaneous velocity of $15 \mathrm{~cm} / \mathrm{s}$. Therefore, the frame rate of the clinical ultrasound image system should be high enough to avoid phase jump errors due to displacement exceeding the threshold. The causes of the phase jump need to be further investigated in future study. The phase jump error is reflected in the quadrature detection as a sudden jump in the phase difference between two consecutive frames along the time axis. As the displacement estimation is calculated from the phase difference, this represents a discrete change in the observed displacement along the time axis.

An issue with the phase jump error is the corruption of the succeeding displacement plot of the signal. The displacement estimate calculated by the quadrature detection is an 
accumulated sum of the instantaneous displacement between consecutive frames. Therefore, a phase jump error between any frames is accumulated and propagated to the displacement estimate at all later time instances. This error propagation may be the reason why the offset persists in the distance plots of the phantom surface in Figure 5.3 and Figure 5.5. The phase jump error at a particular time index resulted in an erroneously displacement and was accumulated along the successive time indices. The effect of the phase jump error may also be observed in the Figure 5.4. The phase jump error that occurred at approximately $2 \mathrm{~s}$ affected the remaining vertical displacement estimates from quadrature detection for time period of $2 \mathrm{~s}$ to 5.5

s.

\subsubsection{Moving Average Filter for Random Noise Error Reduction}

In Chapter 4, the application of the moving average filter was proposed for the reduction of random noise to improve the SNR of acquired ultrasonic RF signals. In this section, we also investigate the effectiveness of the moving average filter to reduce the phase estimation errors when there is vertical displacement in a medium during ultrasonic RF signal acquisition. This reduction in random noise may also result in reducing the phase jump errors encountered during the displacement estimation using quadrature detection.

The displacement plot of the phantom that underwent compression by Plexiglas plates after the application of a moving average filter of length 5 frames is shown in Figure 5.6. The estimated distance from ultrasound probe to top phantom surface using quadrature demodulation and cross-correlation is shown in Figure 5.7. It can be seen in Figure 5.7 that the offset value after $4.5 \mathrm{~s}$ decreases when comparing with the result without moving average filtering in Figure 5.3. In addition to reduction of the random noise in the acquired ultrasound RF signal, we also want to investigate if the application of the moving average filter resulted in a reduction in the 
phase jump errors. As phase jump errors do not occur at all depths it is difficult to determine the reduction effectiveness through examination of the accumulated displacement along the time axis. In order to best determine the reduction in phase jump errors the instantaneous displacement between two consecutive frames across the depth axis is examined.

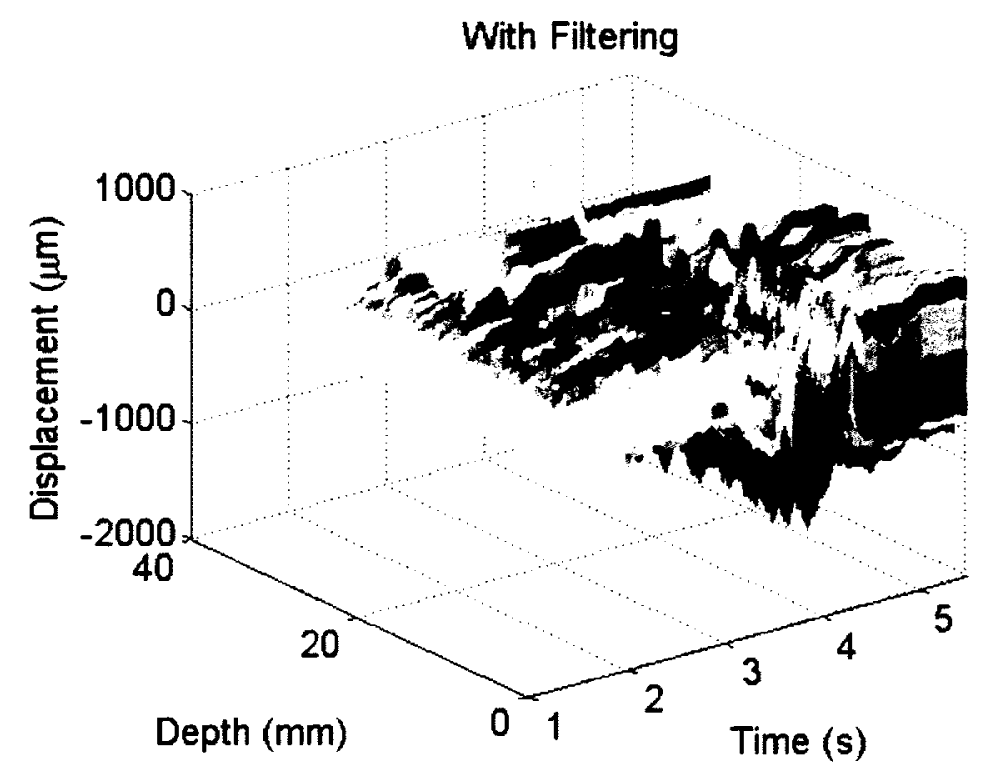

Figure 5.6 Displacement of phantom due to Plexiglas compression with moving average filter 


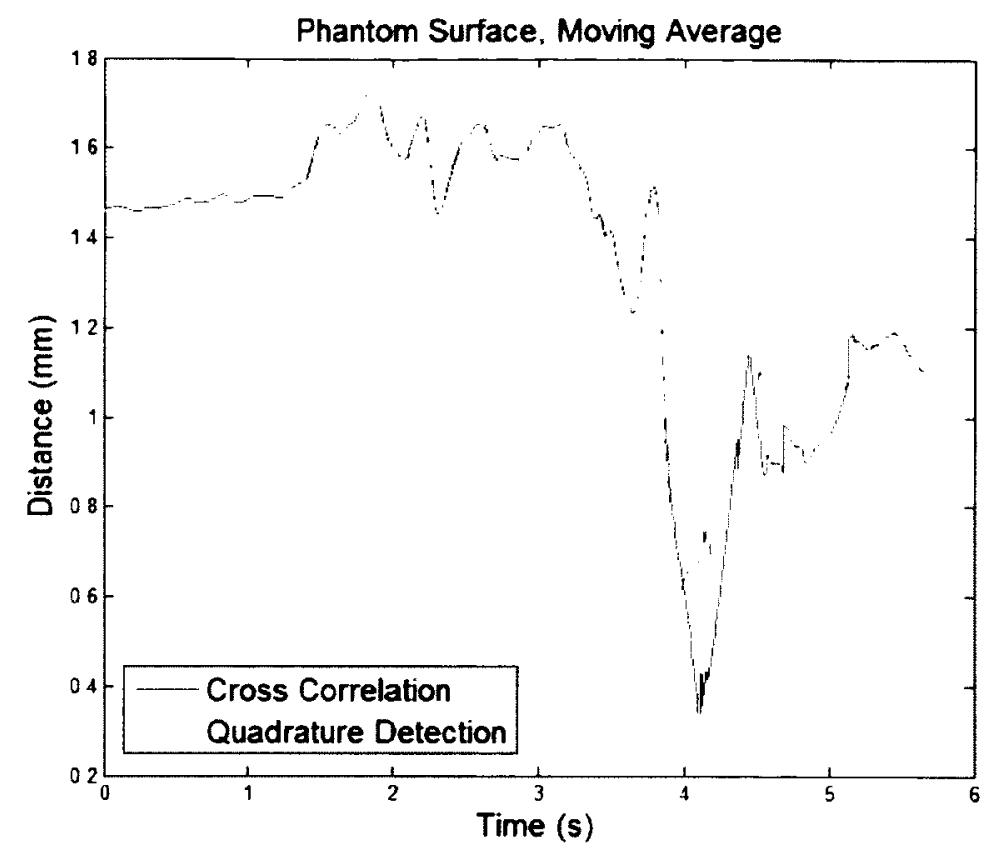

Figure 5.7 Distance of phantom surface distance from ultrasound probe during Plexiglas compression with moving average filter. Cross-correlation estimate is shown in solid blue line, and quadrature demodulation is shown in red dashed line.

The instantaneous displacement across the depth axis obtained from quadrature detection is examined at two particular time instances in the measurement times: 1.) Reference period at $1 \mathrm{~s}$ and 2.) Compression period at 3.9s. The two time indices are selected such that the phantom is static in the reference period, and is undergoing compression in the compression period. The plots of the instantaneous displacement from the original data and after filtering are shown in Figure 5.8 and Figure 5.9 respectively. 

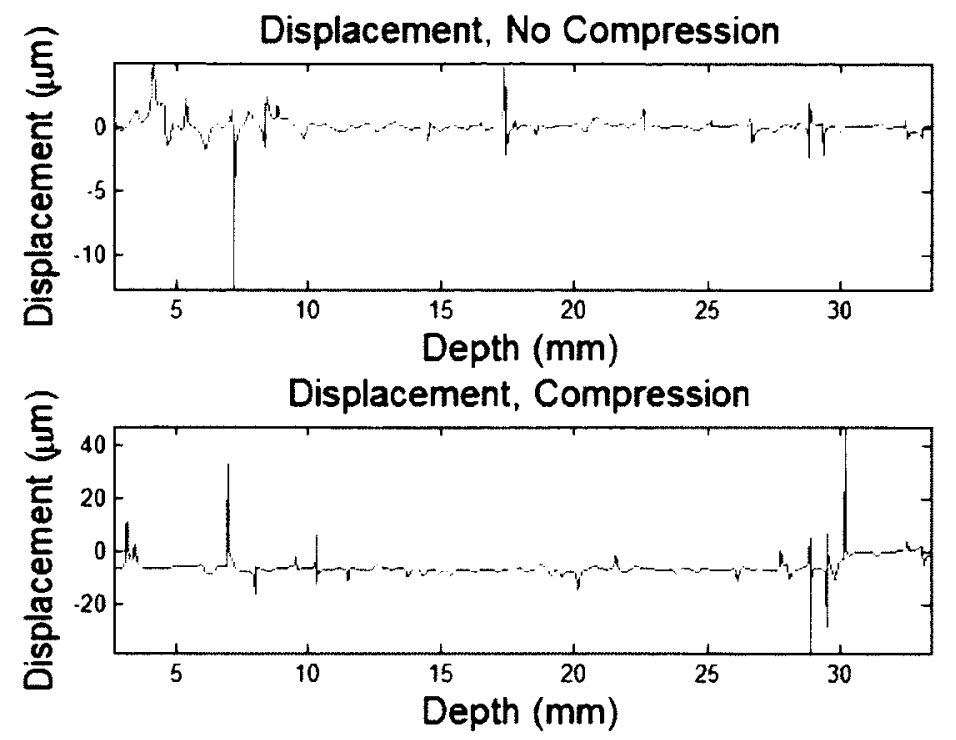

Figure 5.8 Instantaneous displacement across depth by Plexiglas compression without moving average filter. Top plot shows the displacement across depth when the phantom is static. Bottom plot shows the displacement across depth as the phantom is undergoing compression
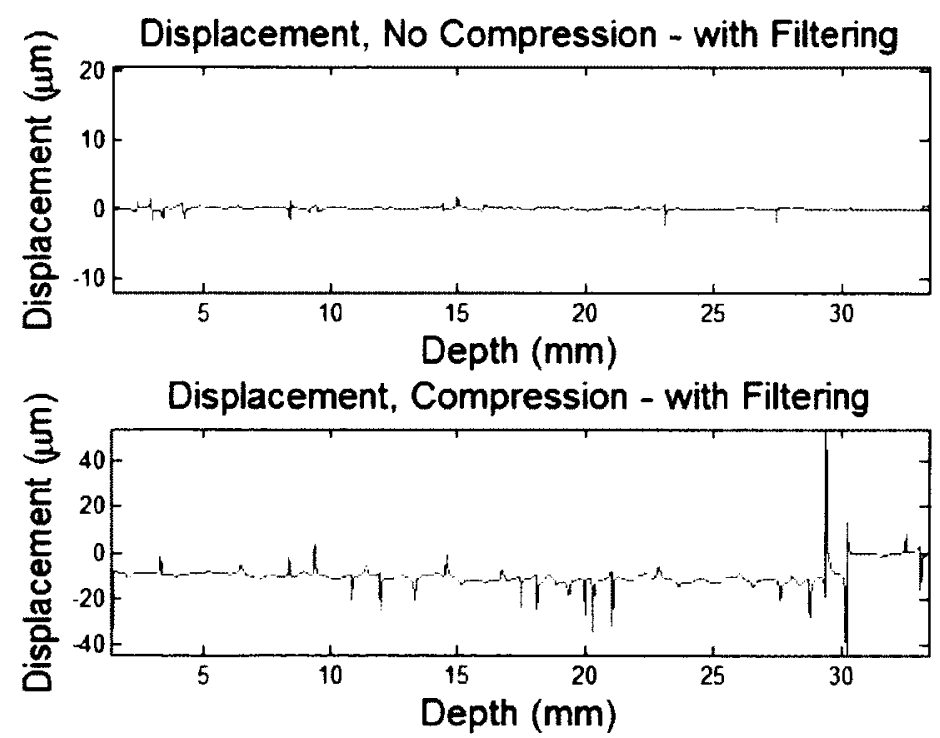

Figure 5.9 Instantaneous displacement across depth by Plexiglas compression with moving average filter. Top plot shows the displacement across depth when the phantom is static. Bottom plot shows the displacement across depth as the phantom is undergoing compression.

The top plot in Figure 5.8 shows the instantaneous displacement between two consecutive frames across depth when the phantom is in static condition and is not experiencing any compression. In the ideal model the phase difference should be zero across the entire depth 
in when the phantom is in static condition. While the top plot in Figure 5.8 shows that the phase difference is approximately zero, there are still variations in the phase between consecutive frames. This estimated phase differences are errors probably due to random noise, or slight probe motion caused by the floor vibration. The bottom plot in Figure 5.8 is the displacement between the consecutive frames across the phantom depth while the phantom is undergoing compression. From this plot it appears that the phantom is undergoing a displacement of about $10 \mu \mathrm{m}$ at this particular time instance. Note that the time difference between the consecutive frames is about $1 \mathrm{~ms}$ between the consecutive frames at the frame rate of $980 \mathrm{~Hz}$. The spikes in both of the plots in Figure 5.8 are likely due to phase jump errors, or inaccuracies in the frequency estimation in the quadrate detection process. Further investigation is required to determine the exact cause.

Figure 5.9 shows the effect of the moving average filter on the instantaneous displacement plots. The top plot in Figure 5.9, where the phantom is in static condition, shows that the moving average filter reduced most of the phase errors. Therefore, the variations observed in the original plot in Figure 5.8 are likely errors due to random noises in ultrasonic RF signals that were reduced by the moving average. Conversely, the moving average filter did not reduce the phase spike errors effectively when there was compression as seen in the lower plot of Figure 5.9. It is likely that one of the primary causes of the phase spikes observed when the phantom was under compression was due to lateral motion. Therefore, the application of the moving average filter would likely not have any effect on those errors, and therefore no improvement was observed in the lower plot of Figure 5.9 compared to Figure 5.8.

The moving average filter is also applied to the data obtained with hand compression of the phantom. The phantom in hand compression was secured more firmly in comparison to 
Plexiglas compression. Therefore, it is expected that there would be less lateral motion effects on the phantom measurement. Thus the application of the moving average filter is expected to have accuracy improvement on the estimated displacement.

The estimated displacement from the quadrature detection technique for the manual hand compression of the phantom is shown in Figure 5.10. Similar to the Plexiglas compression results it is difficult to identify the improvement from filtering in this figure format. The vertical distance from ultrasound probe to surface after filtering is shown in Figure 5.11. The application of the moving average filter resulted in a better agreement between the vertical distances obtained from the quadrature detection and cross-correlation methods. The improvement consistency between the estimated displacements from the two different methods is likely due to the reduction in phase jump error caused by the random noises in the quadrature detection technique. The vertical distances at $0 \mathrm{~s}$ and $5.5 \mathrm{~s}$ after the filtering are approximately $2.65 \mathrm{~mm}$ and $2.95 \mathrm{~mm}$ for both the quadrature detection and cross-correlation displacement estimates. The displacement estimates with filtering shows a $0.3 \mathrm{~mm}$ change in vertical displacement from the start and end of the measurement period. 


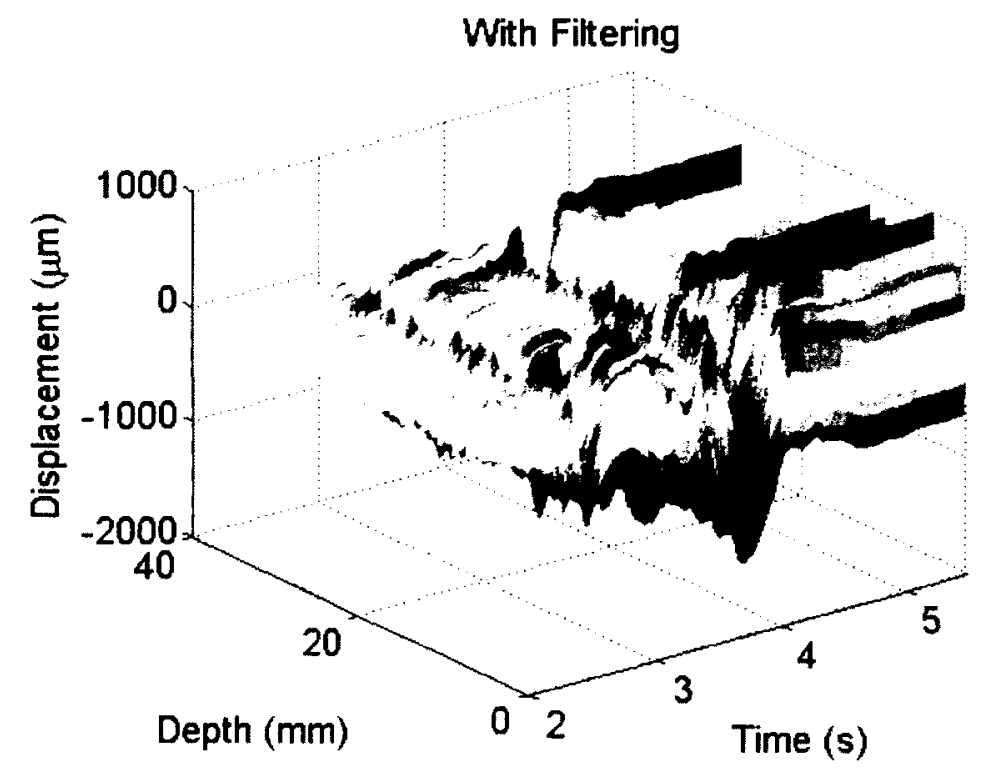

Figure 5.10 Phantom vertical displacement from hand compression with moving average filtering

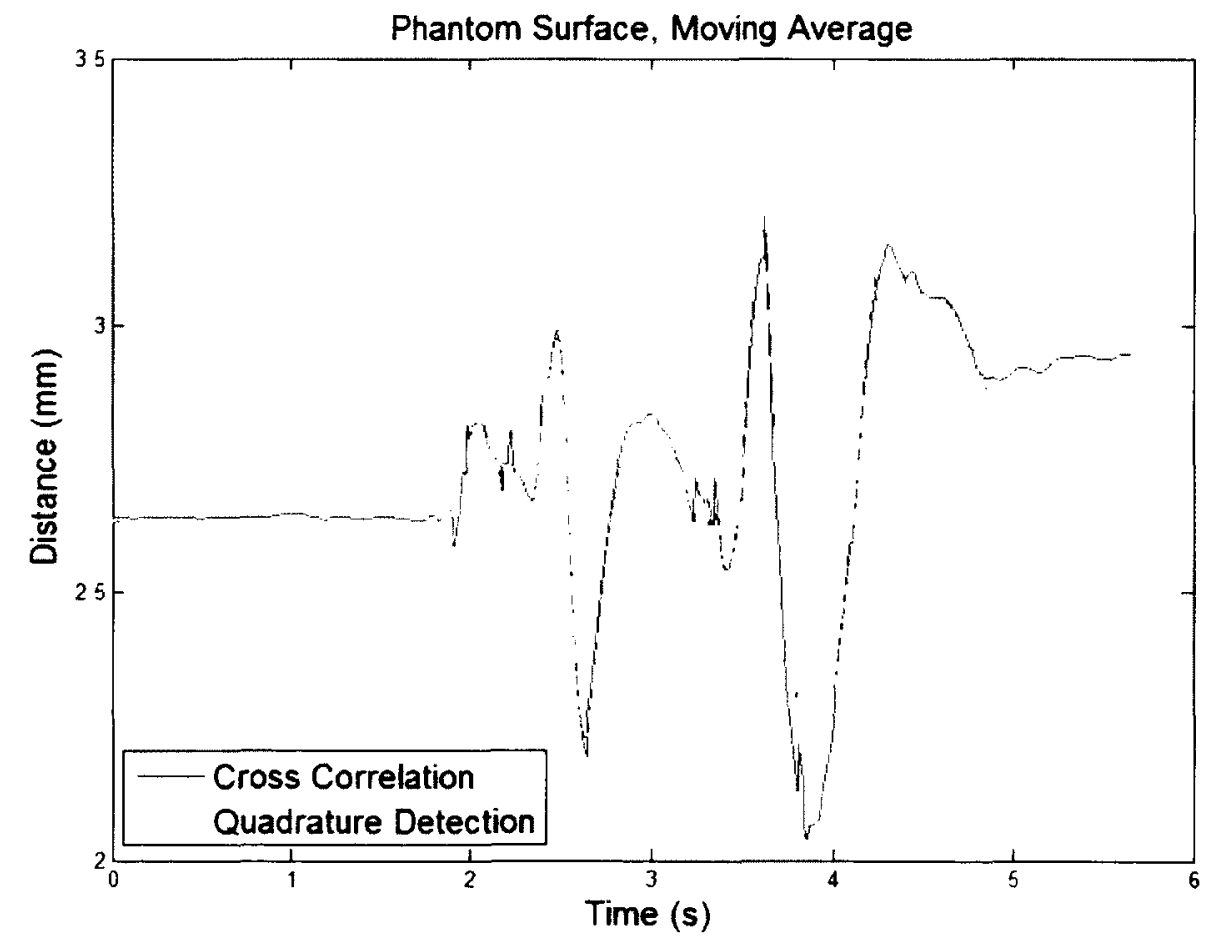

Figure 5.11 Distance of phantom surface from ultrasound probe during hand compression with moving average filtering. Cross-correlation estimate is shown in solid blue line, and quadrature demodulation is shown in red dashed line. 
The plots of the displacement across depth for time instances of phantom in static condition and phantom undergoing hand compression are shown in Figure 5.12. The same data after the application of the moving average filter is shown in Figure 5.13. Similar to the Plexiglas compression data, the moving average filter removed the majority of the phase spike when the phantom is static. In comparison to the Plexiglas plate compression data in Figure 5.9, the moving average filter also resulted in a reduction in the phase jump errors while the phantom is undergoing hand compression. The difference in the results between Figure 5.9 and Figure 5.13 is likely due to the difference in lateral motion amount between the two compression methods. However, compared to when the phantom is in static condition there are still some observable phase spikes when the phantom is undergoing compression. Therefore, there is likely lateral displacement that occurs within the phantom alongside the vertical displacement during compression.
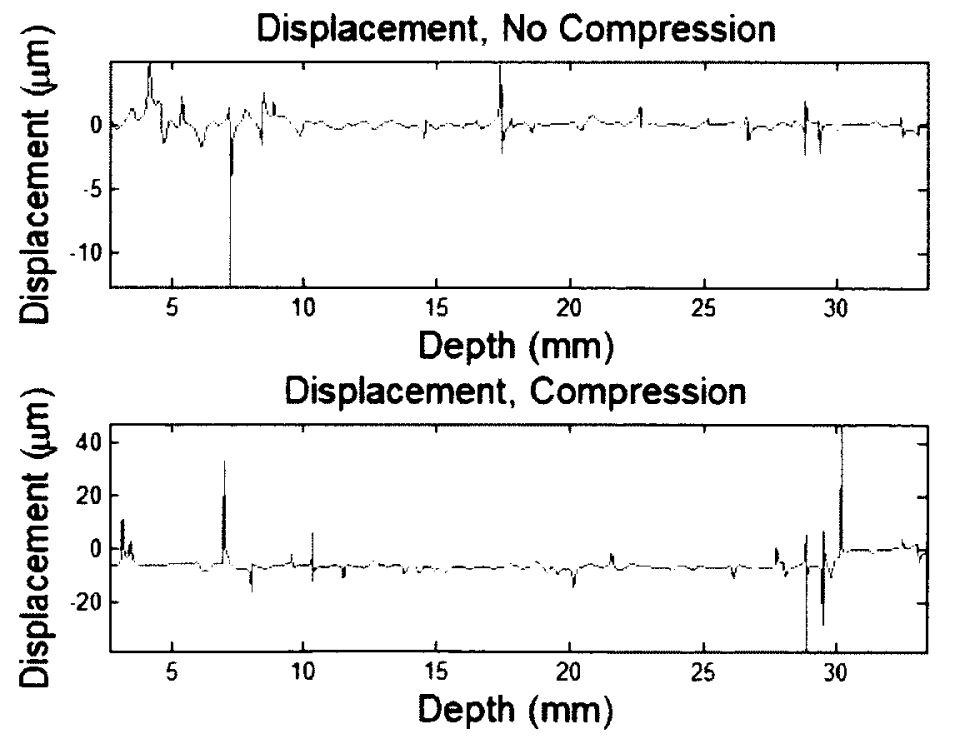

Figure 5.12 Instantaneous displacement across depth by hand compression without mobbing average filtering. Top plot shows the displacement across depth when the phantom is static. Bottom plot shows the displacement across depth as the phantom is undergoing compression. 

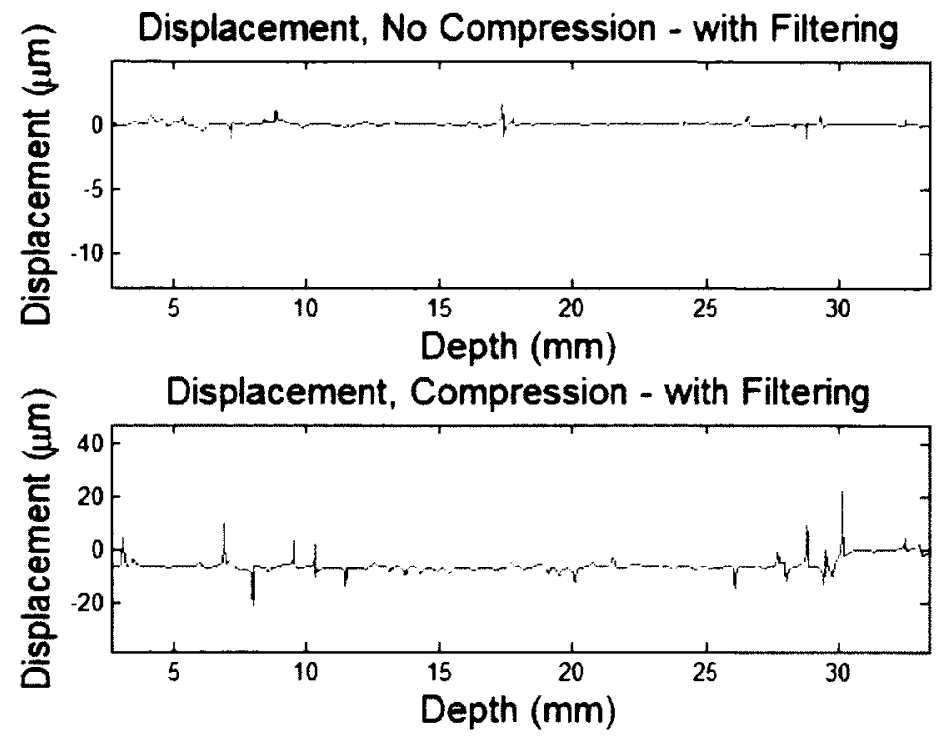

Figure 5.13 Instantaneous displacement across depth for hand compression after moving average filtering. Top plot shows the displacement across depth when the phantom is static. Bottom plot shows the displacement across depth as the phantom is undergoing compression.

From the above results of the phantom simulations, the moving average filter reduces the random noise in the ultrasonic RF signal. This random noise reduction is most apparent in a reduction in instantaneous displacement estimate spikes across the depth axis while the phantom is in static condition, as shown in Figure 5.9 and Figure 5.13. The reduction in phase jump error also improves the consistency between the displacement estimates obtained from the crosscorrelation and quadrature detection technique as shown in Figure 5.11. Based on the results shown in Chapter 4, it is shown that the moving average filter would not improve phase estimation accuracy effectively in the presence of large lateral motion. This expectation is confirmed in the plot of the phantom under Plexiglas compression shown in Figure 5.9. The hand compression of the phantom results in less lateral motion in comparison to the Plexiglas compression 


\subsection{In Vivo Experiments}

In this section the displacement estimation errors are examined in in vivo experiments with a human subject. To measure the muscle displacement in in vivo, contractions of the extensor carpi radialis in a forearm were performed in a water bath. Ultrasound measurements were performed to estimate the vertical displacement of the muscle during isotonic (dynamic) and isometric (static) contractions.

\subsubsection{Experimental Setup and Procedure}

To avoid direct contact between the body and the ultrasound probe and let the body be free to move, a water bath is used to allow ultrasound measurement through water layer between the probing and body surfaces. The muscle of interest is the extensor carpi radialis on the subject's left forearm. The subject of the experiment was a 26 year old male. The measurement setup is shown in Figure 5.14. The subject's left arm is submerged in a water bath. The forearm is placed on the bottom of the bath in a pronated position with the palm towards the container bottom. The ultrasound probe is positioned above the subject's extensor carpi radialis. The ultrasound imaging system is used to record $5.5 \mathrm{~s}$ of M-mode measurements with $1 \mathrm{~s}$ of no movement at the start followed by $3 \mathrm{~s}$ of contractions, and then $1.5 \mathrm{~s}$ of no movement.

Two different types of contractions are performed - isotonic and isometric. In the isotonic contraction the subject contracts the extensor carpi radialis by lifting his palm away from the table without moving the forearm. The extensor carpi radialis is relaxed by placing the palm back on the bottom of the water bath. Over a $1 \mathrm{~s}$ period the palm is raised about $6 \mathrm{~cm}$ and lowered. This rise and lowering of the palm is repeated twice in the $3 \mathrm{~s}$ contraction measured period to produce two isotonic contractions. In the isometric contraction, for the $3 \mathrm{~s}$ period the 
palm is raised and held about $6 \mathrm{~cm}$ away from the bottom of the bath. At the end of the $3 \mathrm{~s}$ period the palm is lowered back to the bottom of the container.

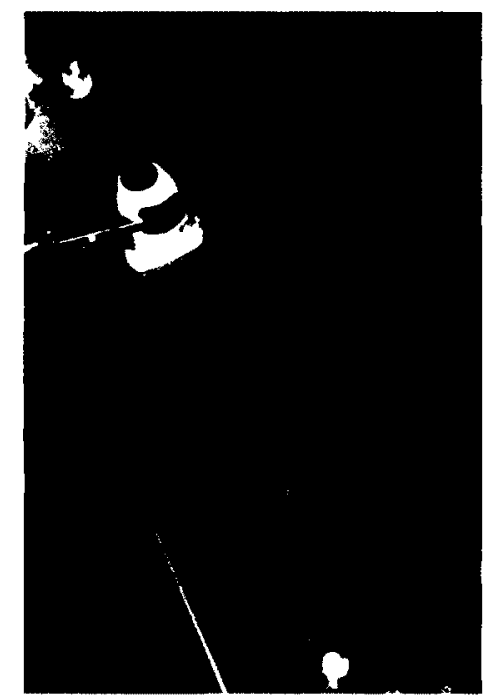

Figure 5.14 In vivo experiment setup

A B-mode image of the cross-section of the forearm was acquired using the clinical ultrasound system during the measurement setup to properly align the probe with the desired measurement area. The B-mode image of the left forearm that contains the target measurement area is shown in Figure 5.15. The colors in the plot indicate the strength of the ultrasound echo, as shown in the color bar in the figure. The locations of strong echoes indicated by the light blue are related to the medium boundaries between different tissue types. As the transmitted ultrasound signal travels through the tissues, the weak scattered echoes from scatterers are observed as indicated by the large dark blue sections of the image. The vertical dotted line in Figure 5.15 indicates the $\mathrm{M}$-mode measurement line. The strong echo from the bone is an important reference signal that will be discussed later in this section. 


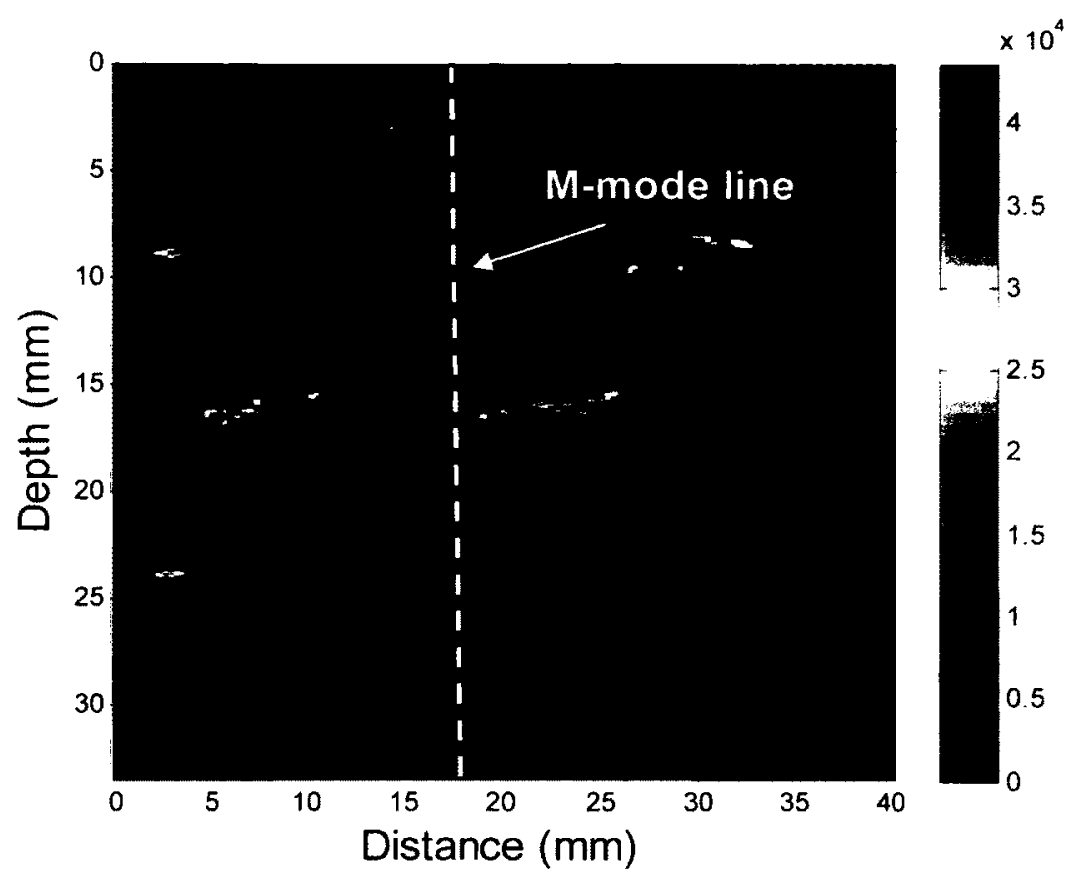

Figure 5.15 B-mode image of left forearm. The target muscle of interest and bone is shown in red. The dashed white vertical line indicates the $\mathrm{M}$-mode measurement view.

\subsubsection{In Vivo Experiment Results}

Quadrature detection is used to estimate the internal tissue displacement during dynamic contraction from the M-mode measurements. The estimated displacements with the dynamic contraction are shown in Figure 5.16. Since the bone surface results in a reflection of the majority of the ultrasound energy, signals beyond the bone surface are most likely noise and unusable. Similarly, the water layer between the probe and arm serves as an ultrasound coupling agent is not relevant to our investigation. The plot in Figure 5.16 therefore only shows the ultrasound RF signal between the arm and the bone surfaces. From the plot, the reference period with no contraction is in the first $1 \mathrm{~s}$ and contraction period is from $1 \mathrm{~s}$ to $4 \mathrm{~s}$ where the two displacement peaks are observed at approximately $2 \mathrm{~s}$ and $3.5 \mathrm{~s}$ corresponding to the muscle contraction. 
As discussed in the previous chapters, one of the issues encountered in muscle monitoring are vertical and/or lateral motions of the probe and/or subject. In in vivo experiments, the subject may move globally in lateral and/or vertical directions relative to the ultrasonic probe due to muscle contraction. These motions result in phase changes of the received ultrasound RF signal therefore result in erroneous internal tissue displacement estimates. However, it is difficult to quantify the global motion, and in particular the lateral motion, that was observed in the measurement. With no prior knowledge of the actual vertical displacement within the muscle it is impossible to separate the errors due to lateral motion with actual vertical displacement.

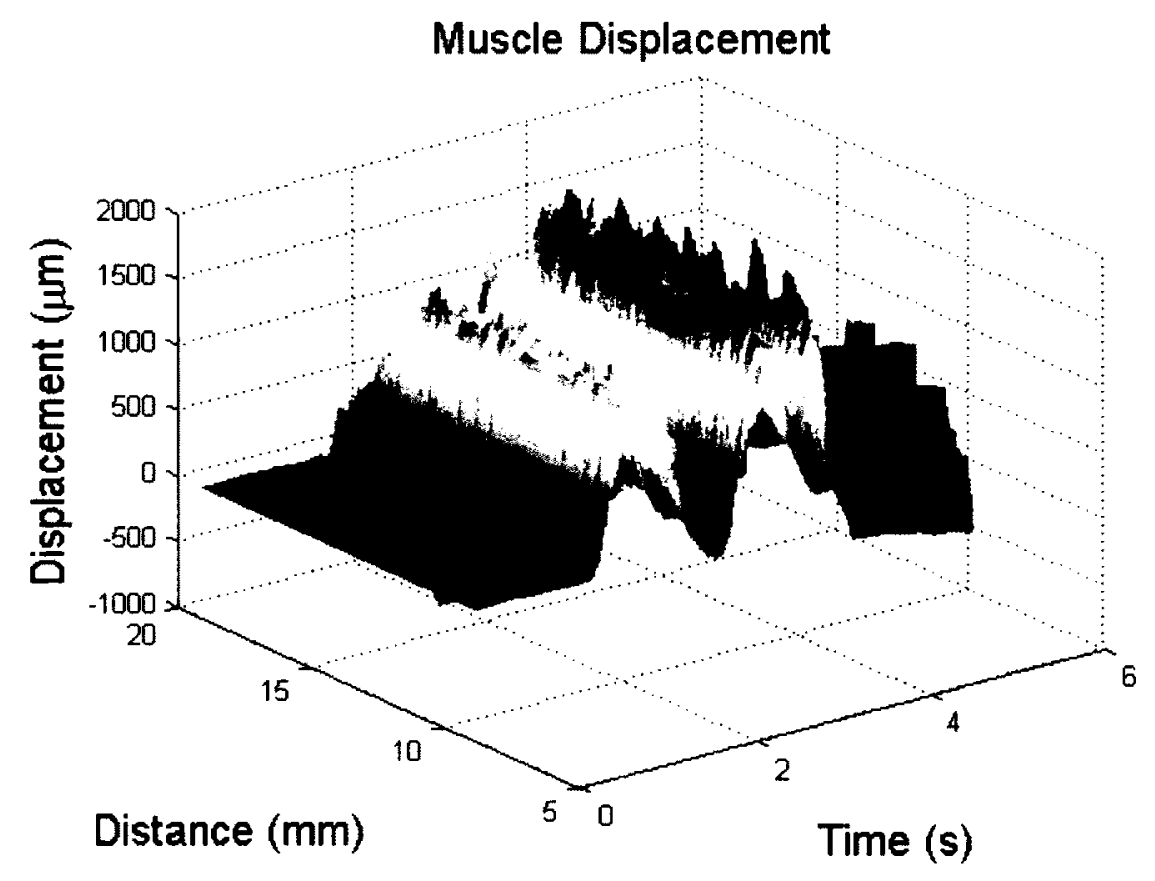

Figure 5.16 Muscle vertical displacement during dynamic contraction

While the effect of lateral motion cannot be compensated for methods have been proposed to mitigate errors in vertical displacement estimation due to global vertical motion. To remove the displacement measurement errors caused by subject vertical movement, the bone 
echo subtraction technique is proposed to compensate the estimated vertical displacements [58]. The bone echo subtraction technique utilizes the displacement observed at the bone to compensate for subject movement. Since the bone is rigid, muscle contraction does not cause deformation of the bone or displacement within the bone. Therefore, any vertical displacements observed at the bone surface are due to subject movement. The vertical displacement measured at the bone using echoes from the bone surface can then be subtracted from the vertical displacements estimated in the tissue to compensate for vertical displacements caused by the subject motion.

Figure 5.17 shows the result of the bone subtraction on the quadrature detection vertical displacement estimates for the dynamic motion experiment presented in Figure 5.16. The removal of the vertical motion error results in a smoother muscle displacement curves during the dynamic contraction. The effectiveness of the bone subtraction is more evident in the results at a particular depth of $15.0 \mathrm{~mm}$ shown in Figure 5.18 as an example. Both quadrature detection and cross-correlation vertical displacement estimates are shown for comparison purposes. The top plot shows the displacement at this depth before bone subtraction, and the bottom plot shows the displacement after the bone subtraction. The bottom plot after bone subtraction in Figure 5.18 is smoother relative to the top plot, and more accurately presents the increase and decrease in displacement due to the muscle contraction. From the results shown in Figure 5.18 a positive displacement is observed at the depth of $15 \mathrm{~mm}$ during muscle contraction. After bone subtraction, it is expected that the displacement observed after $4 \mathrm{~s}$ would be $0 \mathrm{~mm}$ since the muscle returns to rest condition. However, a negative displacement value of $-200 \mu \mathrm{m}$ to -400 $\mu \mathrm{m}$ is observed at the rest state. This non-zero displacement is likely due to lateral motion of the 
arm during contraction. This result in measurement of a slightly different section of the muscle while at rest state at $4 \mathrm{~s}$ to $5.5 \mathrm{~s}$ compared to the initial measurement at $0 \mathrm{~s}$ to $1.5 \mathrm{~s}$.

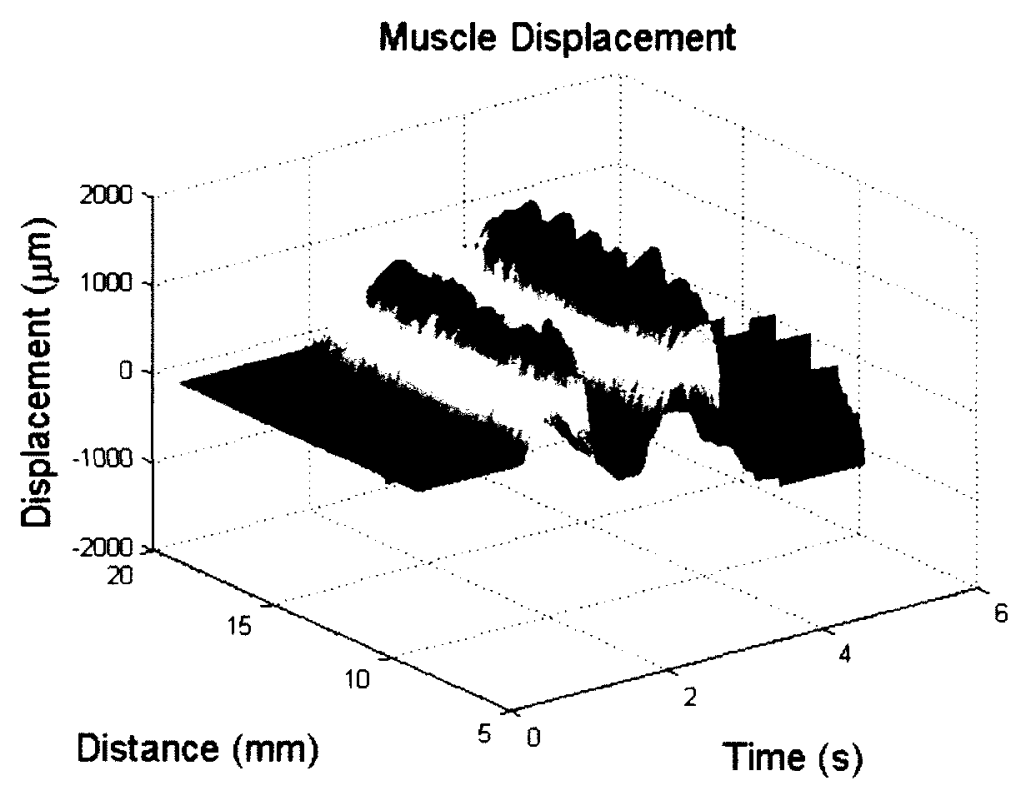

Figure 5.17 Muscle vertical displacement during dynamic contraction after bone subtraction

In the isometric (static) contraction, the muscle contraction is maintained without changing the muscle length. Therefore, the lateral motion of muscle relative to a ultrasonic probe in the isometric contraction could be less than that in the isotonic contraction discussed above. 

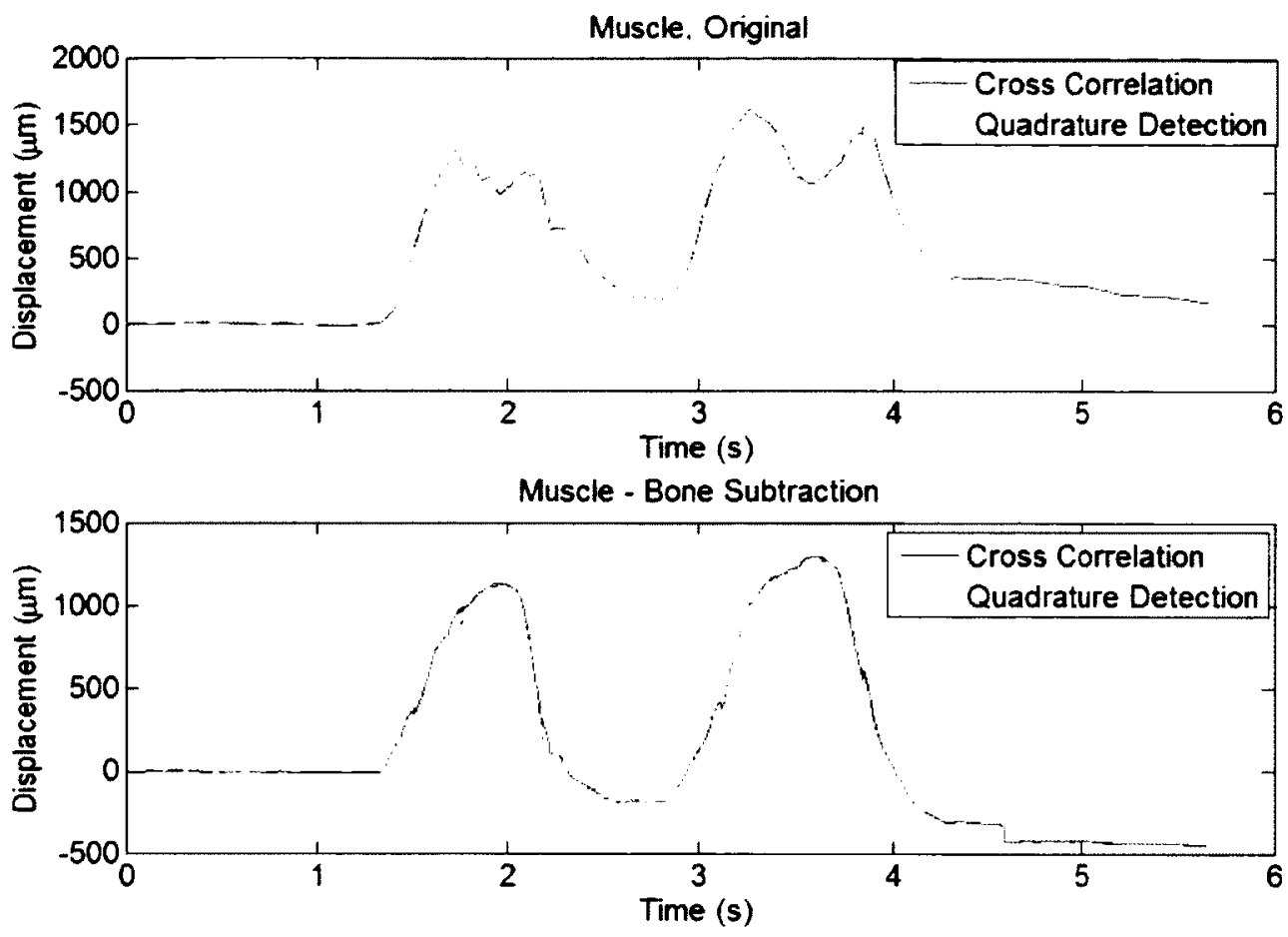

Figure 5.18 Estimated displacement at depth of $15.0 \mathrm{~mm}$. Top plot shows the original data without bone subtraction. Bottom plot shows the data after bone subtraction. Cross-correlation estimate is shown in solid blue line, and quadrature demodulation is shown in red dashed line.

The vertical displacement estimates during isotonic contraction from quadrature detection is shown in Figure 5.19. Bone subtraction is applied to reduce the vertical motion errors, with the results given in Figure 5.20. The vertical displacement without and with the bone subtraction from quadrature detection and cross-correlation at a particular depth of $13.2 \mathrm{~mm}$ is shown in Figure 5.21. 


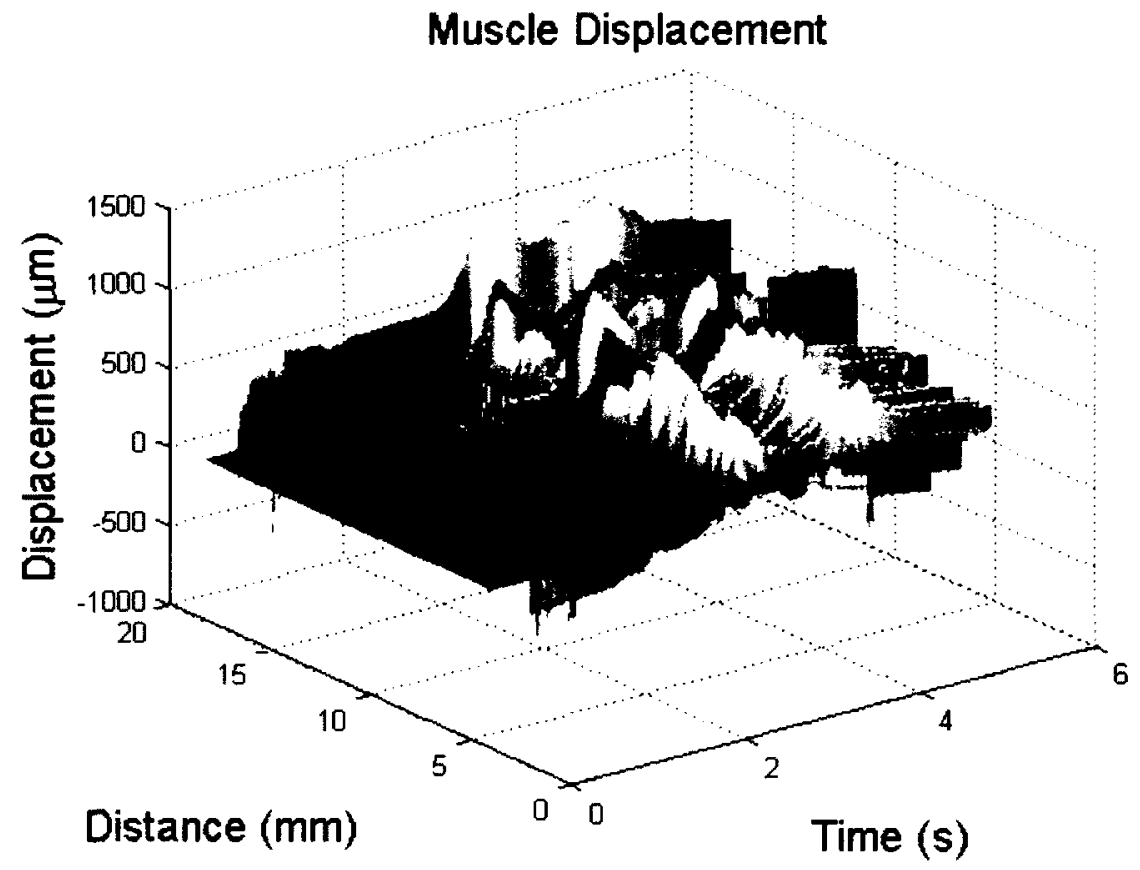

Figure 5.19 Muscle vertical displacement during isometric contraction without bone subtraction.

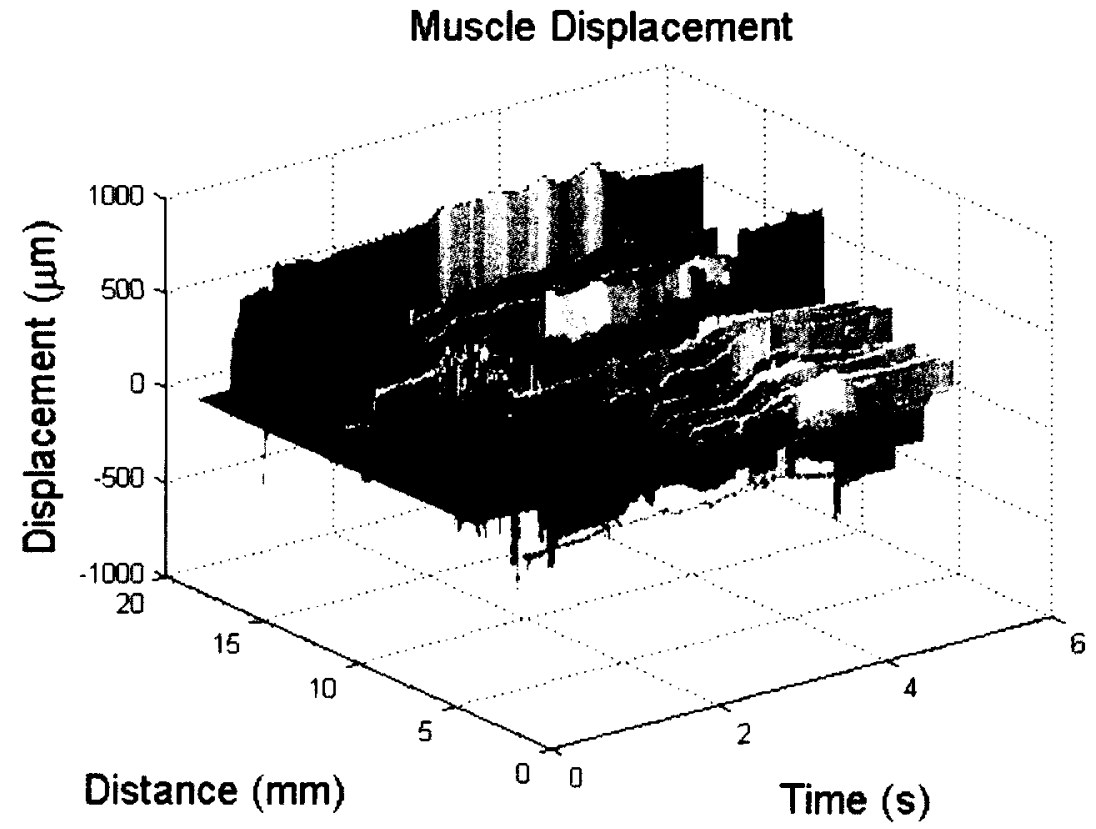

Figure 5.20 Muscle vertical displacement during isometric contraction with bone subtraction 

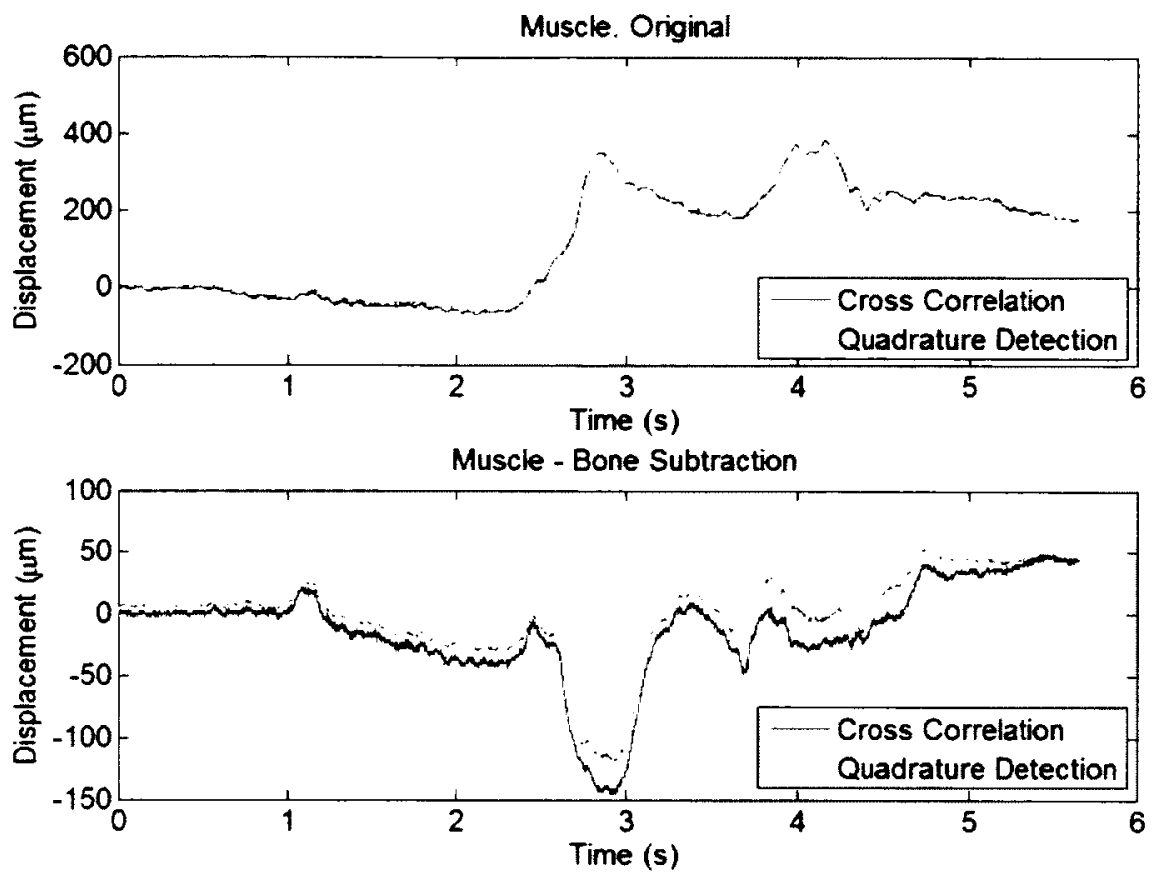

Figure 5.21 Estimated displacement at depth of $13.2 \mathrm{~mm}$ during isometric contraction. Top plot shows the original data without bone subtraction. Bottom plot shows the data after bone subtraction. Cross-correlation estimate is shown in solid blue line, and quadrature demodulation is shown in red dashed line.

The raw data without bone subtraction would indicate that two muscle contractions occurred at $3 \mathrm{~s}$ and $4 \mathrm{~s}$. However, with bone subtraction the distance is relatively constant throughout the entire measurement period. A negative displacement was observed after bone subtraction at $3 \mathrm{~s}$ contrary to the positive displacement observed in the plot of the raw data. The decrease of the distance is expected since the muscle contraction moves the internal tissue towards the ultrasonic probe. In comparison, the muscle demonstrated a positive displacement in the isotonic contraction experiment as shown in Figure 5.18. The muscle contraction resulted in increase and decrease in thickness in different areas of the muscle. While an effort was made to have a same measurement area between experiments, it is likely that due to subject movement there was a change in the location measured. This change in location may also be the cause of the observed displacement at only $3 \mathrm{~s}$ compared to expected interval from 1.5-4 s. The 
ultrasound measurements of the dynamic and isometric contractions were therefore possibly at different locations.

With repeated trials of the experiment it is likely that there would be slightly different results. The design of the experiment provides the ability to accurately observe the larger overall behaviour of the muscles from the measurements. However, it is expected that the minutiae of the results would differ due to the effects of lateral motion and random noise. While the effect of lateral motion is random and difficult to determine, it is likely that there are particularly common lateral movement associated with the specific conditions employed in the experiment. Further study is necessary to determine the impact of lateral motion on the reproducibility of the experimental results.

\subsubsection{Moving Average Filter for Random Noise Error Reduction}

The moving average filter is applied to reduce the random noise in the acquired ultrasound RF signal and remove the phase spike errors due to random noise in the quadrature detection process. From the phantom simulation results in Section 5.1.3 the moving average filter was shown to remove the phase spike errors that were likely due to random noise, but was ineffective on errors that were likely due to lateral motion.

The in vivo displacement measurements with the isotonic contraction over the entire measurement period after the application of the moving average filter is shown in Figure 5.22. Bone subtraction was applied after the filtering to remove the vertical motion errors. The plot of the estimated displacement from the quadrature detection technique at a specific depth of $15 \mathrm{~mm}$ is shown in Figure 5.23. Since phase jump errors do not occur at every depth, it is difficult to ascertain the improvement from the application of the moving average filter from examination of a single depth. Therefore, similar to the phantom simulation results in Section 5.1.3 to 
investigate the effect of the moving average filter, the displacement at particular time indices along the depth axis is examined.

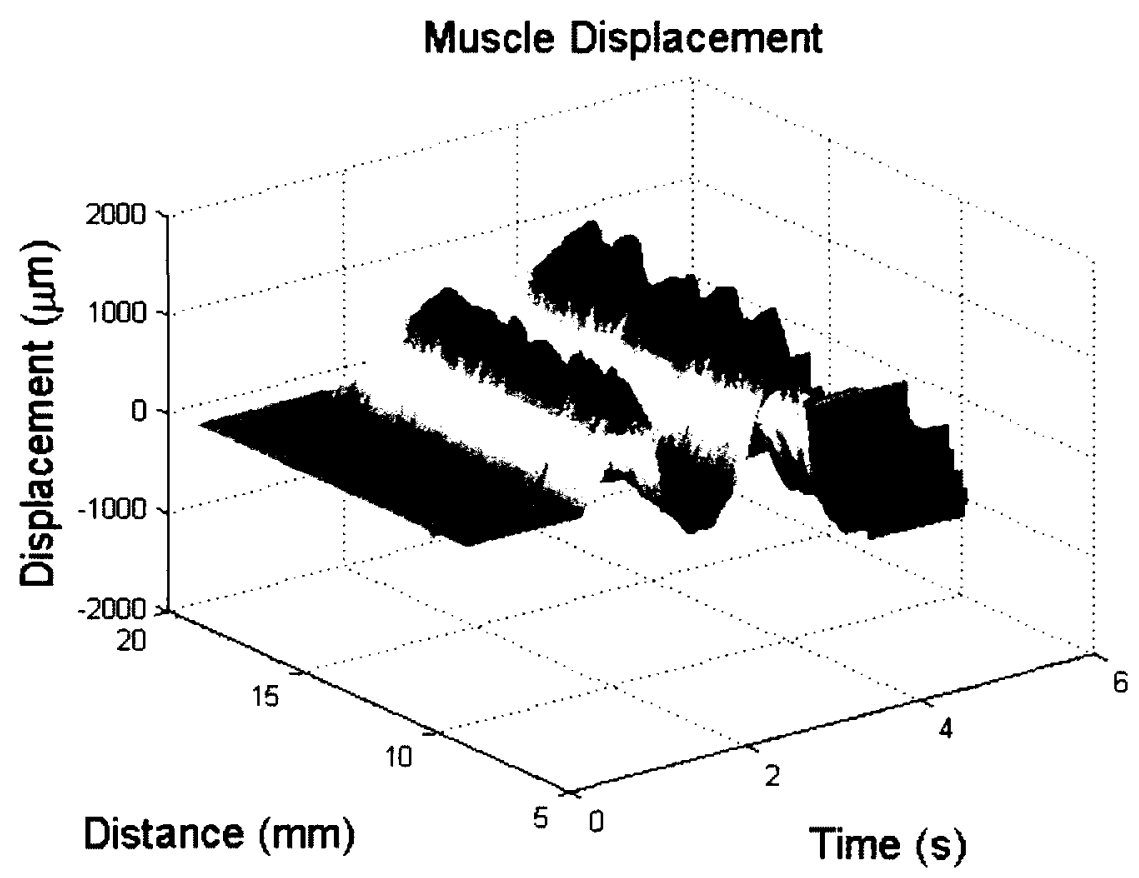

Figure 5.22 Estimated displacement from quadrature demodulation from dynamic contraction experiment after bone subtraction and moving average filter

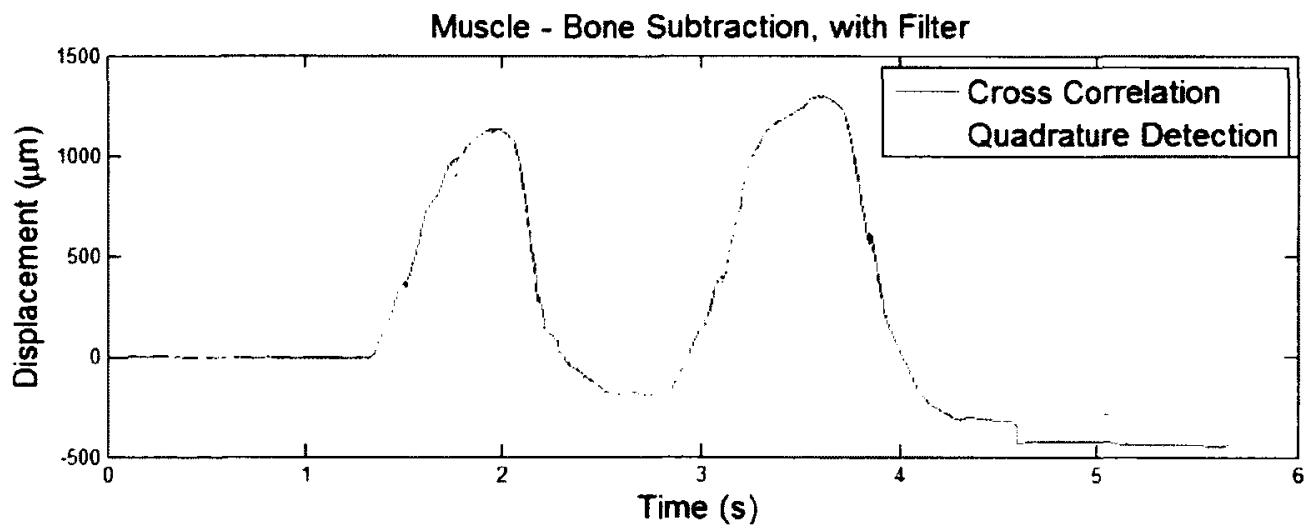

Figure 5.23 Displacement at muscle-muscle interface during dynamic contraction after bone subtraction and moving average filter. Cross-correlation estimate is shown in solid blue line, and quadrature demodulation is shown in red dashed line.

In Figure 5.24 and Figure 5.25 the plots of the instantaneous displacements between two consecutive frames (the time difference is about $1 \mathrm{~ms}$ between the consecutive frame at the 
frame rate of $980 \mathrm{~Hz}$ ) without and with the moving average filter are shown for the isotonic contraction at $0.8 \mathrm{~s}$ (top) and $3.8 \mathrm{~s}$ (bottom). Each figure shows the displacement along the depth when the muscle is at rest and when the muscle was undergoing contraction.

Similar to the phantom simulation results, the reduction in the random noise due to the moving average filter resulted in a reduction in the phase spike errors, as shown in the top plots of Figure 5.24 and Figure 5.25 when the muscle is at rest (or in static condition). Similarly the phantom compression results, the moving average filter did not result in an effective reduction in the phase spike errors when the muscle was contracting (or compressed). It is likely that the phase spikes are due to lateral motion and therefore would not be removed effectively by the moving average filter. In both the phantom simulation and in vivo experiment, contraction/compression of the muscle/phantom results in a lateral motion alongside the vertical displacement. The errors due to this lateral motion are therefore likely the primary cause of the phase spikes in the bottom plots of Figure 5.24 and Figure 5.25.
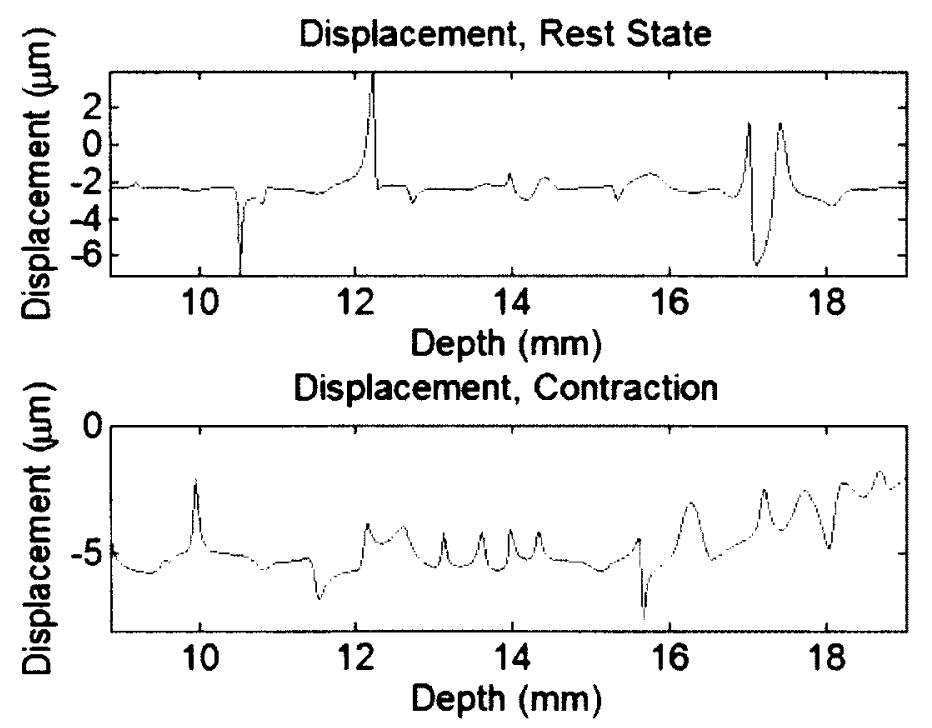

Figure 5.24 Instantaneous displacement estimate from quadrature demodulation for isotonic contraction experiment. Top plot is at a time index where the muscle was at rest. Bottom plot is when the muscle is in the process of being contracted. 

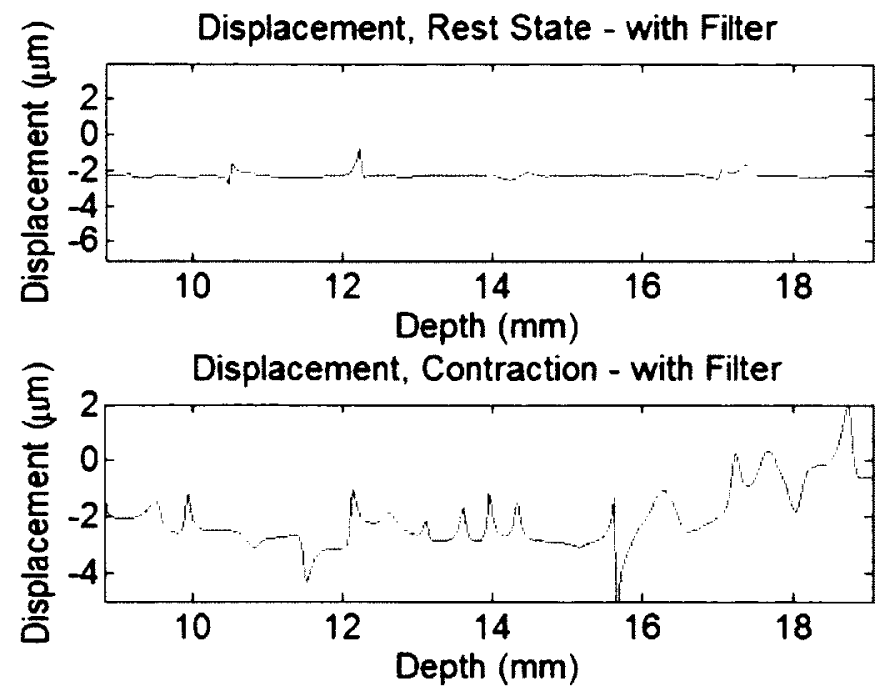

Figure 5.25 Instantaneous displacement estimate from quadrature demodulation for isotonic contraction experiment after moving average filter. Top plot is at a time index where the muscle was at rest. Bottom plot is when the muscle is in the process of being contracted.

The effectiveness of the moving average filter on the isometric contraction results is also investigated. The estimated displacement from quadrature detection of the isometric contraction after the moving average filter and bone subtraction is shown in Figure 5.26. The plots of the vertical displacement observed at $13.2 \mathrm{~mm}$ from cross-correlation and quadrature detection are shown in Figure 5.27. Similar to the isotonic contraction results discussed above, the distance plot in Figure 5.23 reveal little improvement due to the application of the moving average filter. Therefore, the plots of the instantaneous displacements between two consecutive frames without and with the moving average filter are shown for the isometric contraction at $0.6 \mathrm{~s}$ (top) and $2.6 \mathrm{~s}$ (bottom) are shown in Figure 5.28 and Figure 5.29. 


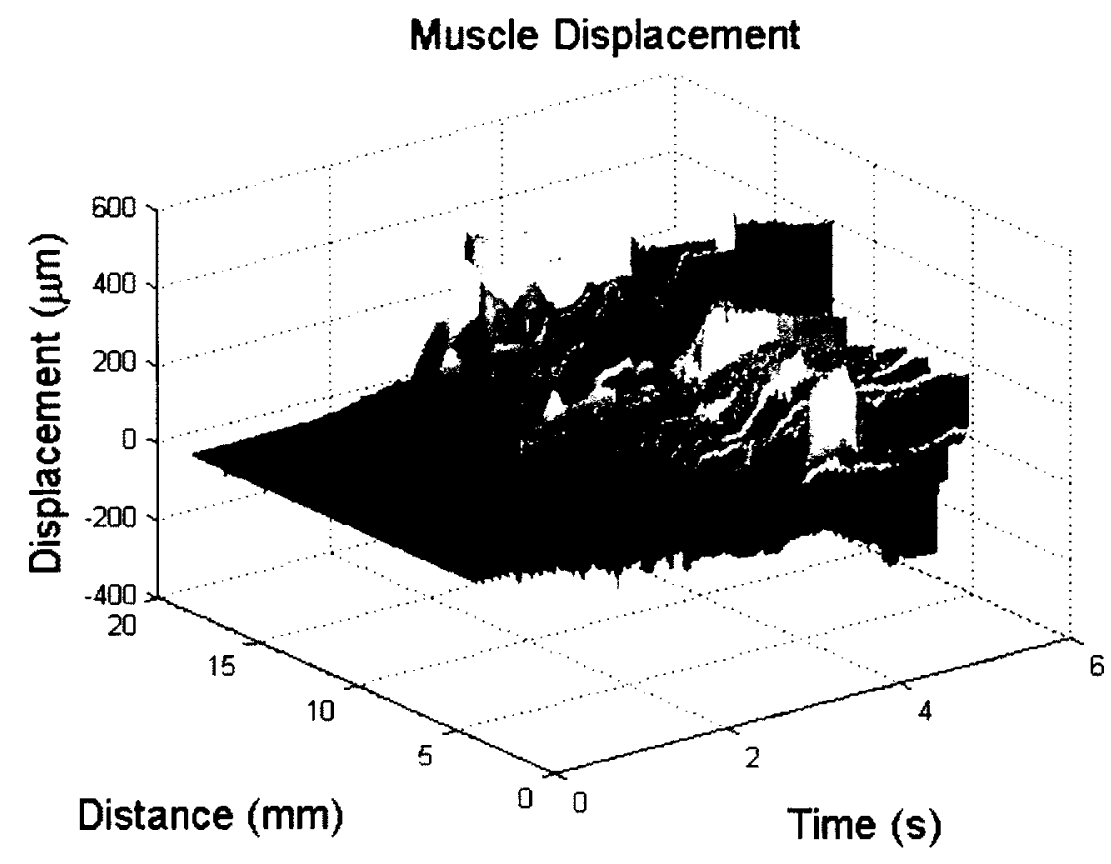

Figure 5.26 Estimated displacement from quadrature demodulation from isometric contraction experiment after bone subtraction and moving average filter

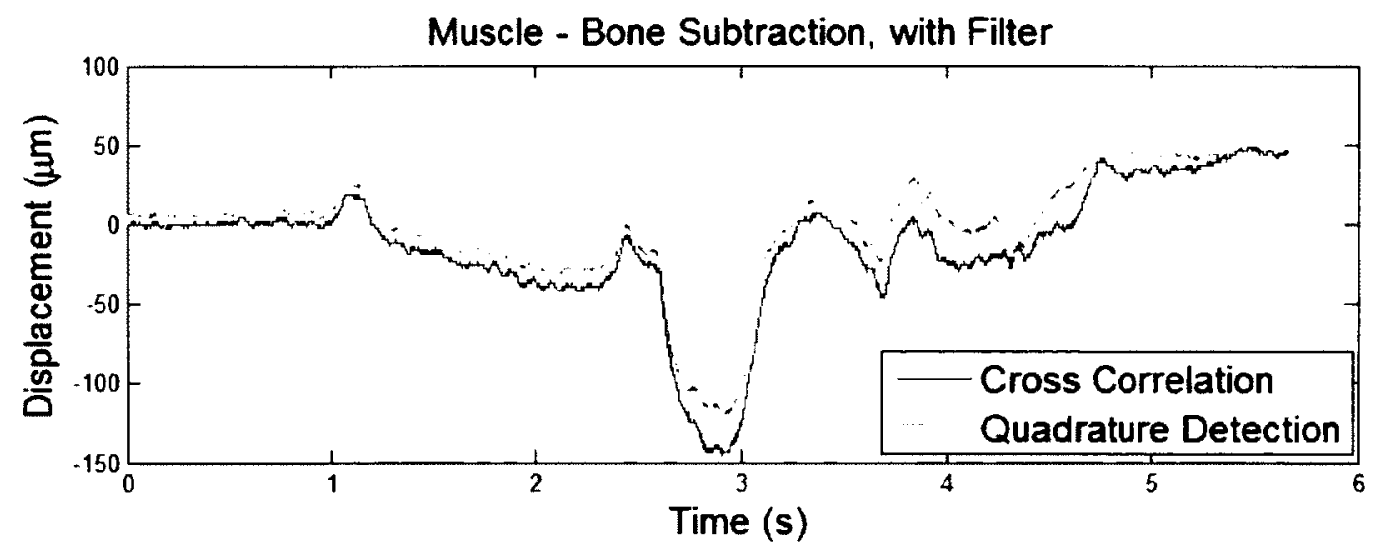

Figure 5.27 Displacement at muscle-muscle interface during isometric contraction with bone subtraction and moving average filter. Cross-correlation estimate is shown in solid blue line, and quadrature demodulation is shown in red dashed line.

Similarly to the phantom simulation and isotonic contraction results, the random noise reduction from the moving average filter resulted in a reduction in the observed phase spike errors while the muscle was at rest. However, the isometric contraction results shown in Figure 
5.29 also reveal that the application of the moving average filter resulted in the removal of the phase spike errors while the muscle was contracted.

The difference between the isometric contraction and the isotonic contraction is the smaller lateral motion of the muscle in the isometric contraction. In the isometric contraction the muscle is held contracted and therefore there should be very little lateral movement. The cause of the phase spike errors in the isometric contraction shown in Figure 5.28 is most likely random noise. Therefore, the moving average filter effectively reduces the phase spike errors, as seen in Figure 5.29.

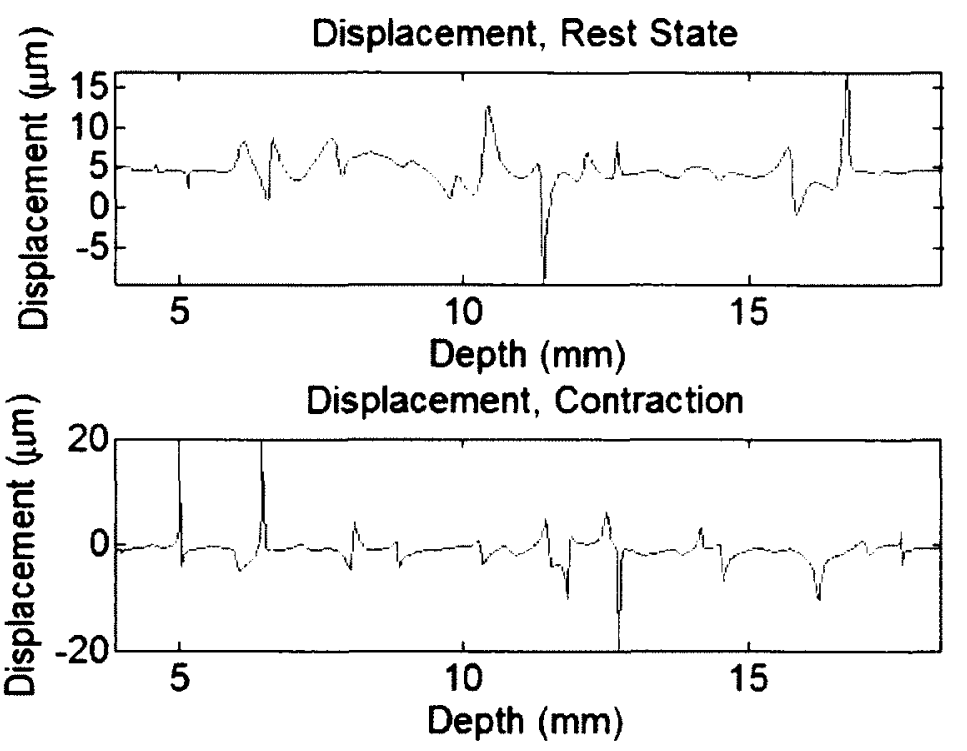

Figure 5.28 Instantaneous displacement estimate from quadrature detection for isometric motion experiment. Top plot is at a time index where the muscle was at rest. Bottom plot is when the muscle is in the process of being contracted. 


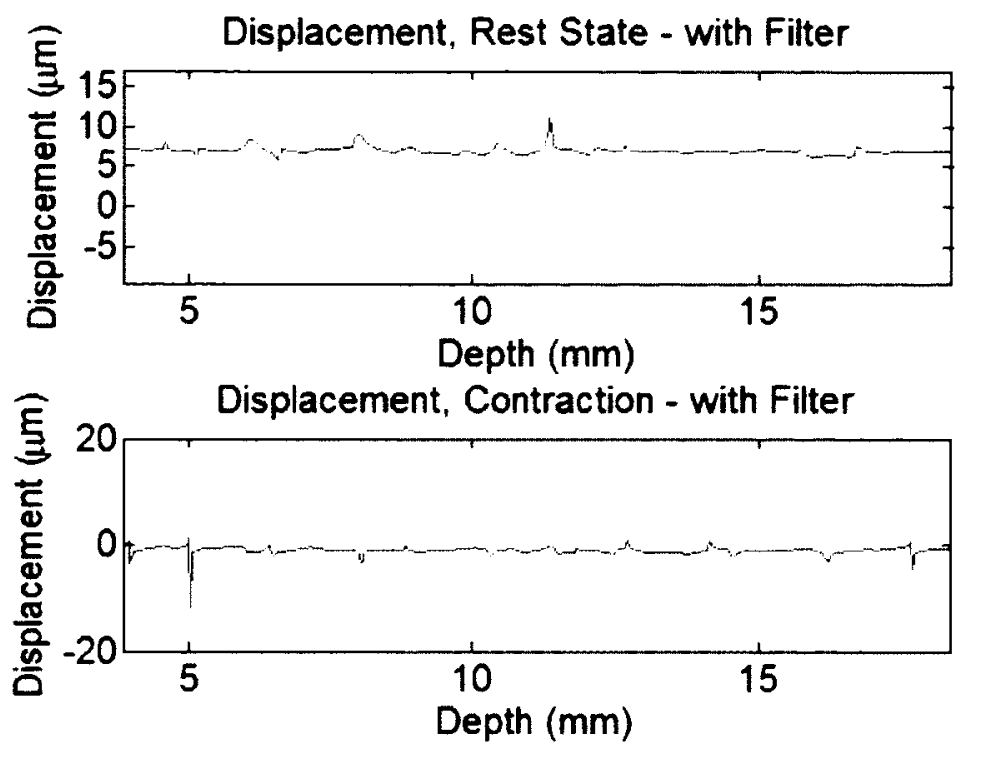

Figure 5.29 Instantaneous displacement estimate from quadrature detection for isometric motion experiment after moving average filter. Top plot is at a time index where the muscle was at rest. Bottom plot is when the muscle is in the process of being contracted.

From the in vivo experiment data it was shown that the moving average filter was able to reduce the phase spike errors that are mainly due to random noise. This appears to occur when the muscle is at rest, or in an isometric contraction. However, when the primary cause of the phase spike appears to be lateral motion the application of the moving average filter does not demonstrate effective improvement in the measurements. The lateral motion appears to be the primary cause of the phase spike errors when the muscle is undergoing isotonic contraction. 


\section{Conclusion and Future Work}

This concludes the thesis and provides a summary of the results. Suggestions for future research are also presented.

\subsection{Overview Conclusion}

The thesis presented an investigation of the effect of lateral probe motion on phase sensitive displacement estimation using ultrasound. The ultrasonic beam width of the ultrasound clinical imaging system used in this thesis was measured to determine the measurement area and for use in numerical simulations. Model-based numerical simulation software was developed and applied alongside phantom simulations to investigate the errors that resulted from lateral probe motion on a static object. A moving average filter was proposed to reduce noise without exacerbating any errors due to the probe motion. Further phantom simulations and in vivo measurements were used to examine the errors due to lateral motion in measurement of nonstationary objects. The impact of the moving average filter was examined on phantom simulation and in vivo experiments to investigate the improvement in measurement accuracy.

It was shown that lateral motion distances below $10 \mu \mathrm{m} /$ frame produced negligible errors in the estimated displacement at the measured SNR levels with the conditions employed in the numerical simulations. An increase in lateral motion distance beyond $10 \mu \mathrm{m} /$ frame resulted in a corresponding increase in the error observed in the displacement estimate from the acquired ultrasound signal. This behaviour continues until the lateral motion distance reaches a threshold where the displacement cannot be determined from the phase sensitive method due to phase wrapping. The proposed moving average filter reduced the random noise in the ultrasound measurement without compounding the errors associated with the lateral motion. This resulted 
in a decrease in observed estimated displacement error at all measured SNR levels for lateral motion distances of $1 \mu \mathrm{m}$ and less. For lateral motion distances of $10 \mu \mathrm{m}$ and above, the moving average filter reduces the estimated displacement error for smaller SNR levels. However, for larger SNR the moving average filter showed no improvement since the lateral motion is more dominant error source than the random noise. The phantom and in vivo experiments demonstrated that the moving average filter improved the measurement accuracy when random noise was the dominant error source; however it was not able to effectively reduce the errors when lateral motion was the dominant error source.

\subsection{Future Research}

As stated above, the primary intent of this work was to investigate the errors that resulted from lateral probe motion in phase sensitive displacement estimation by ultrasound. The research was done to determine appropriate measurement conditions to ensure accurate results. The following section outlines suggestions for future work.

While this work has covered evaluation on the effect of the lateral probe motion it does not cover methods to compensate for any lateral probe motion present in the acquired ultrasound data. The M-mode ultrasound measurements used in this thesis allows for high temporal resolution measurements. However, compared to B-mode images, M-mode suffers from a smaller measurement area. One of the issues with compensation of lateral motion errors is the loss of information due to the shift of the measurement area. One potential method to compensate for lateral motion errors is the use of a B-mode image captured before data acquisition to recover the lost measurement area. The B-mode image has a larger field of view and may be used to compensate for movement of the M-mode measurement area. 
Another area that warrants further investigation are the phase spikes observed in the in vivo measurements as seen in Chapter 5. It is hypothesized that these phase spikes are caused by phase jumps [65], or inaccuracies and approximations transmitted ultrasound signal frequency used in the quadrature detection technique. However, further investigation is required to determine the actual cause of the observed phase jumps.

Finally, there is work that is required to investigate applications of muscle monitoring. One potential use of ultrasound muscle monitoring is in conjunction with EMG measurements [40]. As described in Chapter 2, EMG captures the electrical activity of the muscle. The combination of ultrasound to measure mechanical activities and EMG can be used to provide a thorough investigation of muscle characteristics for a wide range of applications. Preliminary investigation of two such applications, muscle fatigue and measurement of electromechanical delay, are presented in Appendix A. 


\section{Appendix A: Ultrasound Muscle Monitoring}

The appendix presents the results from a preliminary investigation into the application of vertical displacement estimations from ultrasound measurements. As mentioned in Chapter 2 EMG measures the electrical activity of the muscle. By combining EMG and ultrasound measurements a more through measurement of the muscle characteristic can be obtained.

In this appendix the application of the ultrasound muscle monitoring in conjunction with sEMG for the investigation of fatigue [1] and determination of the electromechanical delay [40] are examined. The fatigue measurement setup and test procedure are presented alongside results and analysis. Afterwards, the electromechanical delay measurement experiment is presented with obtained results and analysis. In both applications, only preliminary results are available as further in-depth investigation was beyond of the scope of this thesis.

\section{A.1 Fatigue Investigation}

Muscle fatigue is the decrease in the maximum force generation or output of the muscle [66]. The detection of muscle fatigue has been used in study of gait analysis [67], rehabilitation [68], and ergonomics [69]. One of the most common methods of fatigue detection is the use of sEMG [70] which can detect the electrical activity associated with muscle contraction as well as the changes in electrical activity as fatigue onsets. In our fatigue investigation sEMG is used to verify the presence of fatigue.

From the in vivo results in Chapter 5, we have shown that ultrasound can detect muscle contraction from the change in estimated vertical displacement. Previous research into fatigue detection from ultrasound has shown that muscle thickness increases with the onset of fatigue 
[1]. The previous research into ultrasound fatigue detection has used B-mode images; in our experiment we hope to replicate the results using M-mode measurements as well as improve measurement accuracy using the moving average filter.

\section{A.1.1 Measurement Setup and Experimental Procedure}

The muscle of interest for fatigue measurement is the extensor carpi radialis of the subject's left arm. Both ultrasound and sEMG measurements were recorded on this muscle during the fatigue experiment. The experiment setup is shown in Figure A.1 showing the placement of the ultrasound probe and sEMG electrodes on the arm.

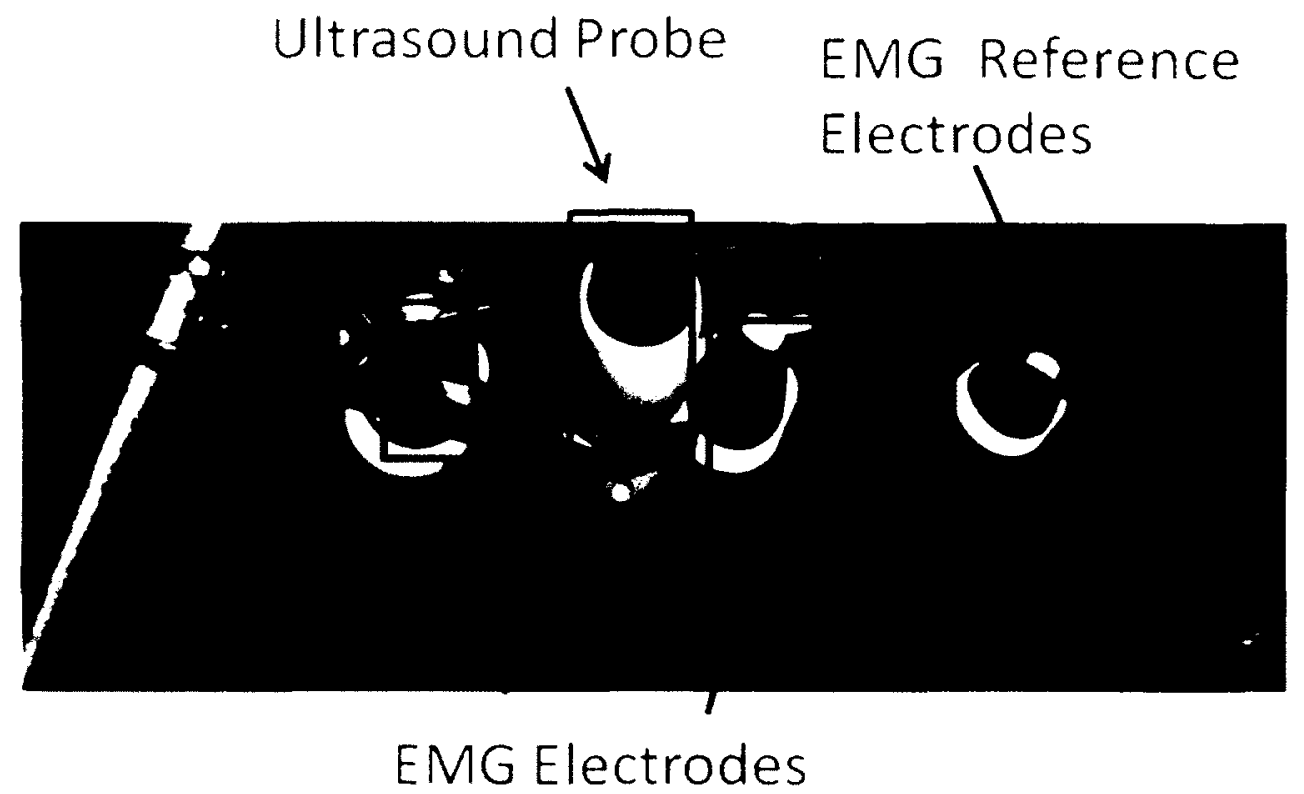

Figure A.1 Experimental Measurement Setup of ultrasound probe and sEMG electrodes to measure the extensor carpi radialis of the subject's left arm

The ultrasound imaging system was also used to synchronously record sEMG alongside ultrasound measurements. The sampling rate of sEMG was the same as the M-mode frame rate. After the skin was prepared using alcohol and Nuprep skin gel, bipolar $\mathrm{Ag}-\mathrm{AgCl}$ electrodes were placed on the extensor carpi radialis with an inter-electrode separation distance of $6 \mathrm{~cm}$. The 
ultrasound probe was placed with a gel couplant between the electrodes. A high-pass Butterworth filter with $2 \mathrm{~Hz}$ cutoff frequency was applied to remove motion artifacts from the sEMG signals due to the dynamic contraction performed in EMD and conduction velocity measurements. The procedure of the fatigue experiment is described as follows.

For the fatigue experiments, the subject's left forearm is moved to allow the hand to hang freely over the table. The subject held a $15 \mathrm{lb}$ dumbbell weight and was asked to hold it for as long as possible while ultrasound and sEMG was measured. Due to hardware constraints, only $5.5 \mathrm{~s}$ of ultrasonic data could be collected continuously at a time. The subject maintained contraction while data were transferred and saved, and then restarted the measurement as quickly as possible. A time-log was obtained from the time stamp of the data file creation.

\section{A.1.2 Preliminary Results and Discussion}

Muscle fatigue is a well-studied subject using sEMG. It has been reported that the median frequency of the sEMG signal decreases while the root mean square (RMS) increases as fatigue onsets [70].

The model of the muscle thickness during fatigue is shown in Figure A.2. The muscle thickness was calculated from the difference in vertical displacement observed at the upper muscle boundary of the extensor carpi radialis compared to the displacement observed at the extensor carpi radialis-bone interface as shown in Figure A.2. If the muscle thickness increases, then the upper muscle boundary is moving away from the muscle-bone interface. Conversely, if the muscle thickness is decreasing than the upper muscle boundary is moving towards the muscle-bone interface. 


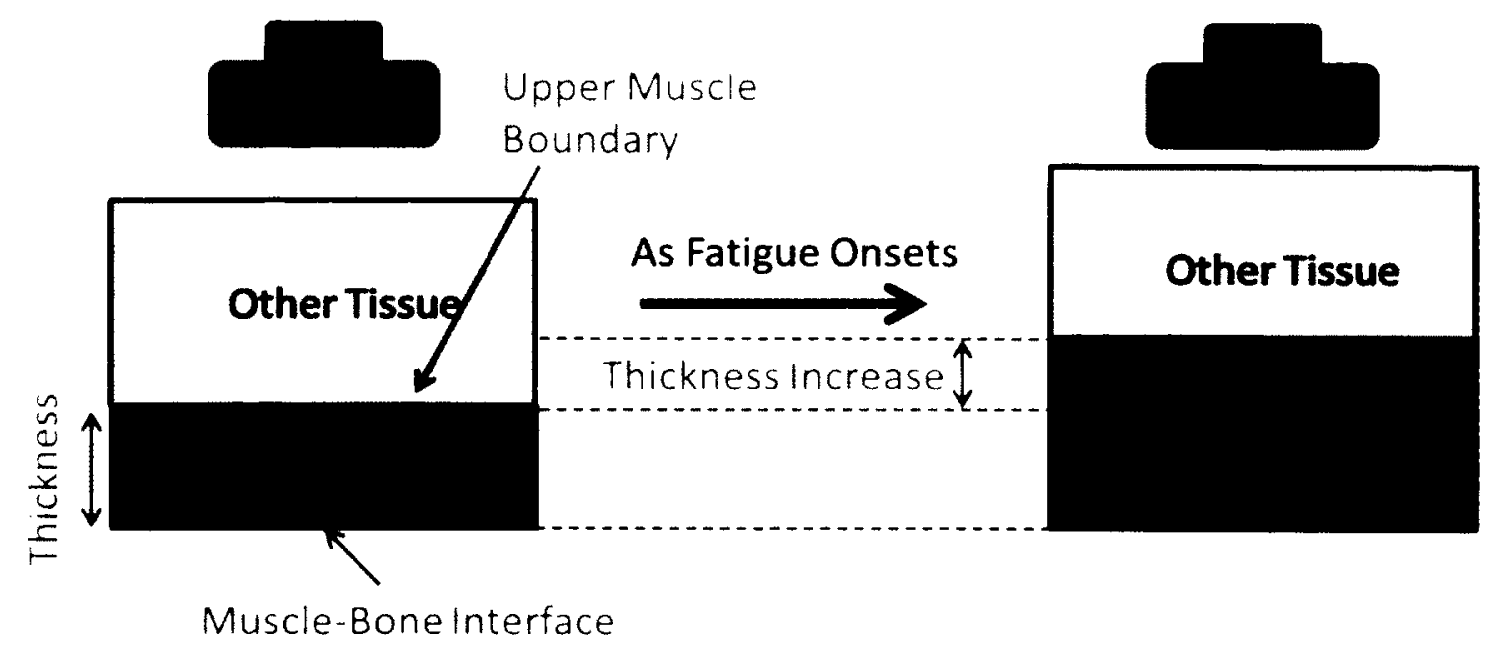

Figure A.2 Fatigue Thickness Model

The muscle thickness results obtained from the muscle fatigue experiment from the ultrasound measurements is shown in Figure A.3. The thickness was calculated as described above with the vertical displacement values obtained from the quadrature detection technique. The $5.5 \mathrm{~s}$ each of recorded data up to the time of $48 \mathrm{~s}$ are shown by the solid lines, while the time gaps indicate the period where no data due the data transmission and storage as described in Section A.1.1. General trends and tendencies may still be observed from these partial measurements. 
15.2

Change in Muscle Thickness

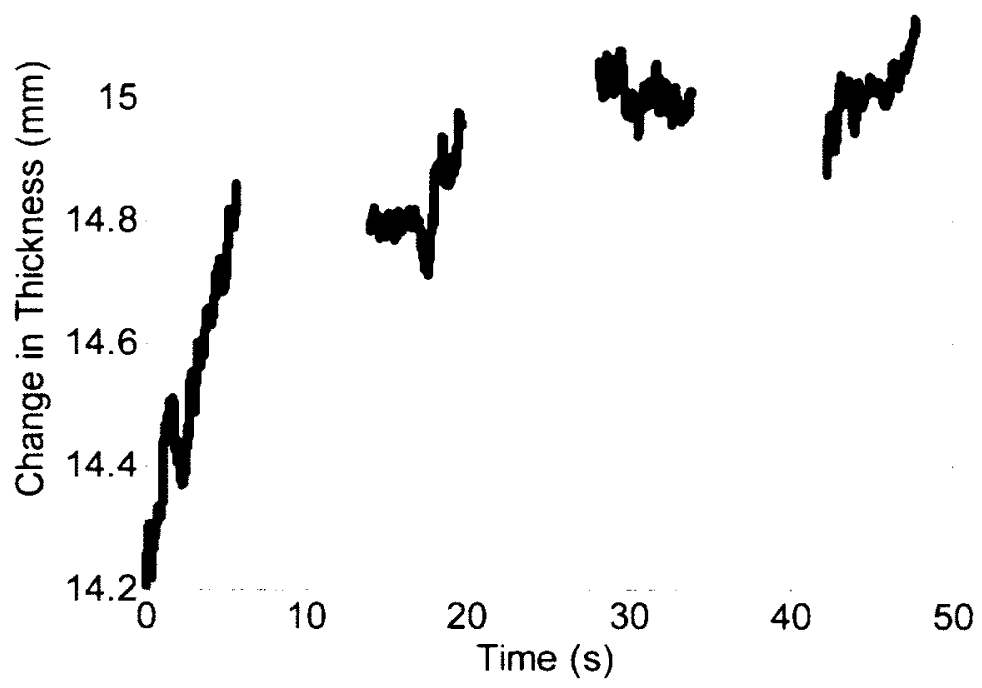

Figure A.3 Change in muscle thickness during fatiguing isometric contraction. The upper muscle boundary located at depth of $13.9 \mathrm{~mm}$

From the result in Figure A.3, it appears that the muscle thickness increased with the onset of fatigue. Verification of the onset of fatigue was performed through analysis of the sEMG signal where the decrease in median frequency from $135 \mathrm{~Hz}$ to $110 \mathrm{~Hz}$ and an 34.5 increase in RMS was observed. The median frequency and RMS was calculated from the entire sEMG signal collected by the clinical ultrasound system during each $\mathrm{M}$-mode measurement period.

One of the limitations with the collection of sEMG with the clinical ultrasound system used in this experiment is the low-pass filtering applied to the sEMG signal. The filter inherent in the clinical ultrasound system limits the spectral content of the sEMG signal to $300 \mathrm{~Hz}$; however the sEMG signal contains valuable information about to $400 \mathrm{~Hz}$. This filter degrades the accuracy of the calculation of the median frequency and sEMG RMS due to removal of the higher frequency content of the sEMG signal. However we are still able to observe the expected downwards shift in the median frequency and the increase in RMS of the filtered signal. 
The muscle thickness showed a rapid increase in the first $5 \mathrm{~s}$ of the isometric contraction as shown in the first measurement period. In the later measurement periods an increase in muscle thickness is still observed, but at a lower rate than the first measurement period. This tendency of increasing muscle thickness during fatiguing isometric contractions has been observed in biceps brachii [1] and gastrocnemius medialis muscle [71] from ultrasound B-mode images. The results obtained from our M-mode experiments are consistent with similar experiment types on different muscles.

The method used to measure the change in muscle thickness in this thesis is based on Mmode measurements. The results shown in Figure A.4 demonstrates that the muscle thickness and associated increase due to fatigue can also be observed in M-mode. In comparison to the Bmode images used in previous research, the calculations using M-mode requires substantially less data. However, the B-mode images have the advantage of accounting for lateral movement during the experiment and are less susceptible to thickness estimation errors due to lateral motion.

While the exact cause of the thickness increase is uncertain, it has been hypothesized that the increase in muscle thickness is due to muscle recruitment. In this experiment, the subject maintained an isometric contraction until failure and the subject could not maintain contraction. The size principle suggests the local recruitment of additional motor units to compensate for the decrease in activated muscle fiber output due to fatigue $[72,73]$. Based on sliding filament theory of muscles, these newly recruited muscle fibers may shorten locally to generate additional force. Since the muscle fiber volume is constant, this local shortening of the muscle fiber is accompanied by an increase in muscle thickness $[74,75]$. Evidence has also shown that the pennation angle of muscle fiber increases in the isometric fatiguing of the gastrocnemius 
medialis muscle [71] and biceps brachii [13]. The pennation angle is the angle of attachment of the skeletal muscle to the bone; therefore an increase in the pennation angle indicates a further contraction of the skeletal muscle.

As discussed in Chapter 5, one source of error in the displacement estimation for quadrature demodulation is phase spike errors. The presence of these phase spikes results in the large discrepancies between the displacement estimations from quadrature detection and crosscorrelation. It was shown that the application of the moving average filter helps mitigate or remove the displacement estimation errors by quadrature detection caused by random noise. The change in muscle thickness with a 5 frame length moving average filter is shown in Figure A.4. Compared to the plot of the original data in Figure A.3, the muscle thickness data with moving average filter shown in Figure A.4 does not show any visible improvements. This shows that the original results are not affected by phase spike errors due to random noise.

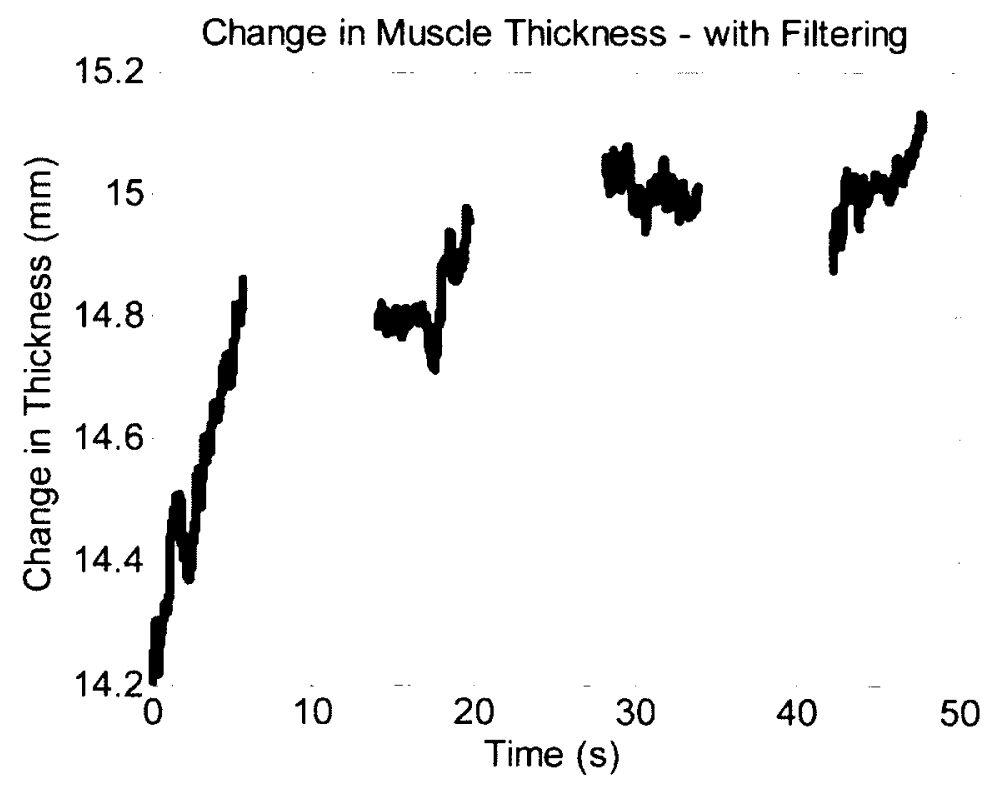

Figure A.4 Change in muscle thickness during fatigue experiment with moving average filter 
One of the difficulties encountered in this study was the synchronized measurement of EMG and ultrasound. While the PICUS ultrasound system could measure sEMG, it was originally intended for electrocardiography measurement. Therefore, it is highly restrictive in terms of sampling frequency and measurement range. While more accurate sEMG measurement hardware is available, it is separate from the ultrasound system. Therefore, an additional synchronization methodology would be required.

\section{A.2 Electromechanical Delay}

The electromechanically delay (EMD) is the time difference between electrical activity of the muscle and the mechanical reaction [76]. It is thought to be a component of stretch reflex, and an indicator of athletic performance [77]. It is useful as an indicator of muscle health, and is known to be modified by parameters such as fatigue.

There are different methods used to measure the EMD each with their own advantages and disadvantages. The requirement of EMD measurement is detection of electrical and mechanical activity during contraction. The electrical activity can be determined by needle or surface electromyography to detect voluntary contractions $[78,79]$ or the use of electrical simulation to induce involuntary muscle contraction $[80,81]$. The mechanical activities of the muscle have been measurement of generated force using force $[80,82]$ or ultrasound to detect muscle contraction $[40,81]$.

The method of EMD measurement used in this thesis is SEMG alongside ultrasound to detect the electrical and mechanical activity generated during muscle contraction. The measurement experiment and experimental procedure are described below, followed by the results and analysis. 


\section{A.2.1 Measurement Setup and Experimental Procedure}

Similar to the fatigue experiment the muscle of interest for EMD measurements is the extensor carpi radialis. The placement of the ultrasound probe and sEMG electrodes is identical to the fatigue measurement setup shown in Figure A.1. The ultrasound imaging system is used to perform synchronized measurement of M-mode ultrasound and sEMG.

In the EMD measurement, the subject performs a sustained isometric contraction of the extensor carpi radialis. With the subject's left forearm placed on the table in a pronated position with palm on the table, the extensor carpi radialis is contracted by lifting the left hand away from the table. The $5.5 \mathrm{~s}$ measurement begins with a $2 \mathrm{~s}$ period of no activity followed by the sustained contraction of the extensor carpi radialis.

\section{A.2.2 Preliminary Results and Discussion}

A result of EMD measurement from the preliminary investigation is shown in Figure A.5. The measured EMG signal is shown by the solid red line while the estimated displacement of the muscle calculated by the quadrature detection technique is shown in the dotted blue line. The EMD is measured as the time delay of the mechanical activity after the associated electrical activity of the muscle. 


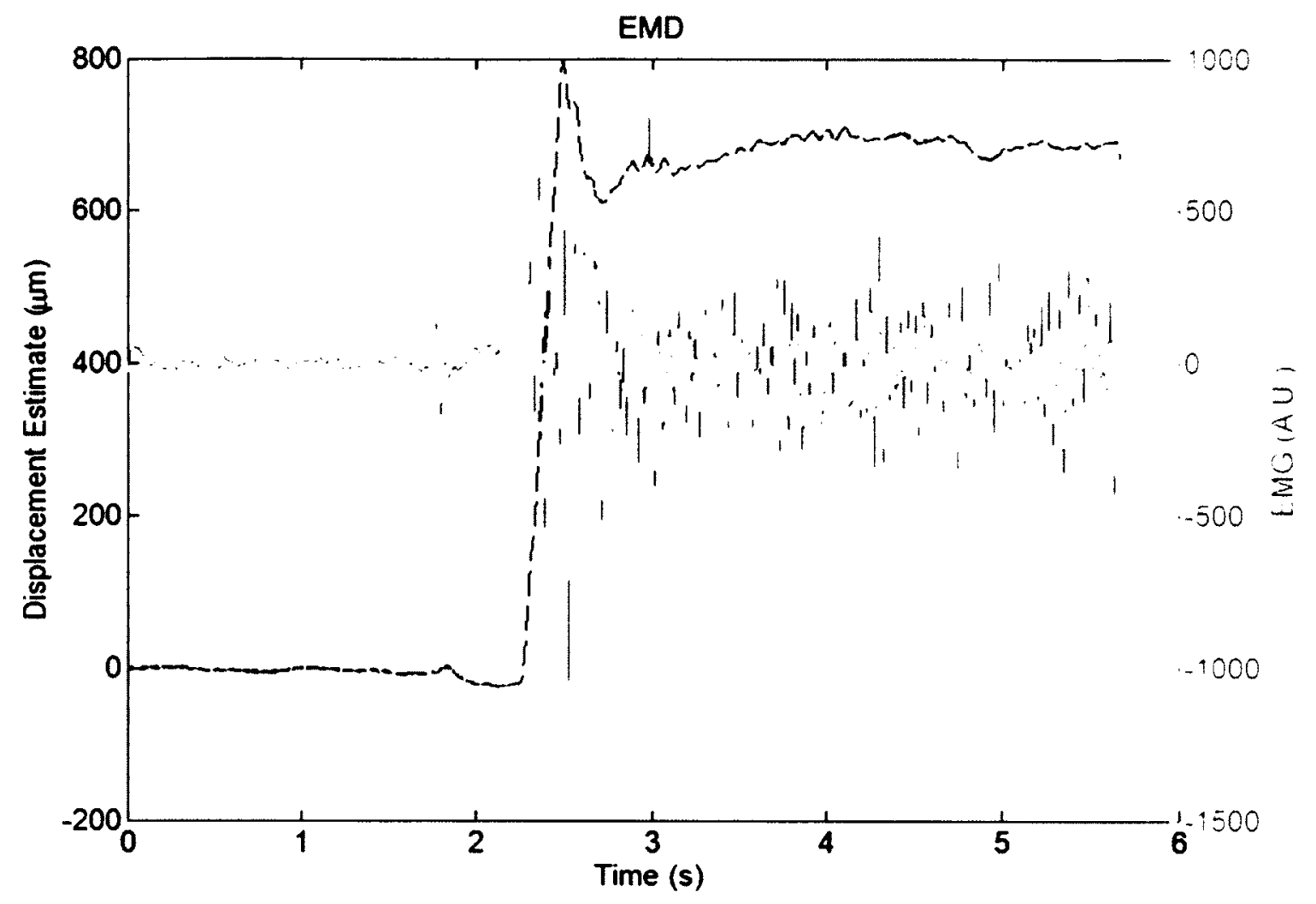

Figure A.5 Electrical and mechanical activity of the extensor carpi radialis during EMD measurement. SEMG results are shown in solid red line, ultrasound thickness measurements shown in the dotted blue line.

The recorded electrical and mechanical activity obtained from sEMG and ultrasound are shown in Figure A.5 as the solid red line and dotted blue line respectively. The mechanical activity is the estimated vertical displacement at $13.86 \mathrm{~mm}$ estimated from the quadrature detection technique. A clear distinction in the muscle activity can be observed between the rest state at the start of the measurement period and the isometric contraction at the end of the measurement period. To ensure the accuracy of the vertical displacement estimate from the quadrature detection technique a moving average filter of length 5 is applied to remove any displacement estimation errors from phase jumps. 
The electrical and mechanical activity with the moving average filter is shown in Figure A.6. From comparison of the results of the original measurements shown in Figure A.5 it appears that there were no phase spike errors due to random noise in the estimation displacements from the quadrature detection technique. The application of the moving average filter was important to ensure no estimated displacement errors due to phase jump errors. The EMD calculation is reliant on accurate detection of start of electrical and mechanical activity; the displacement errors due to the phase jumps may result in inaccurate estimation of initial mechanical activity and therefore determination of the EMD value.

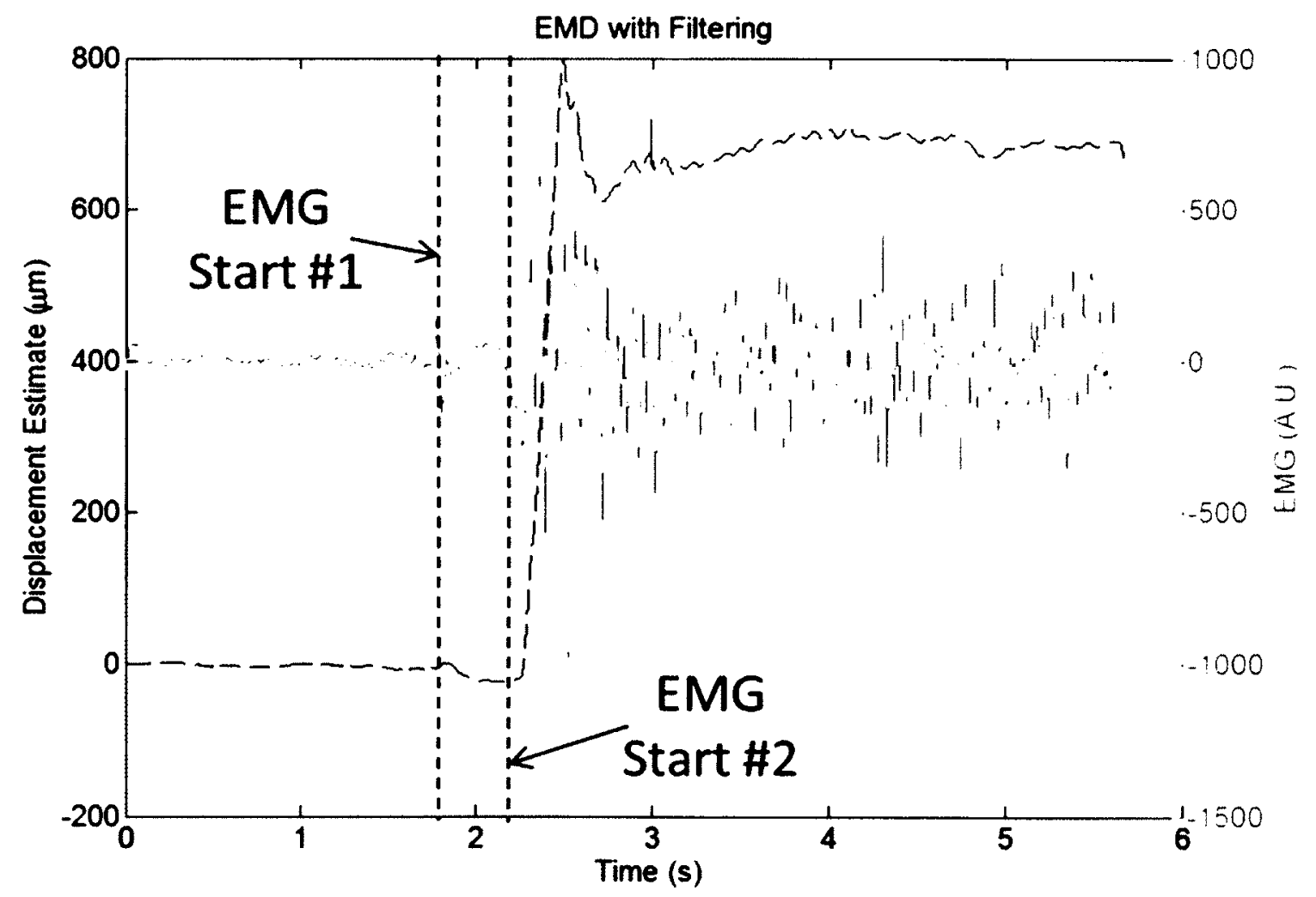

Figure A.6 Mechanical and electrical activity of extensor carpi radialis with moving average filter. Vertical black line shows the two possibilities of initial electrical activity

The primary difficulty encountered with this application is the determination of activity detection for sEMG and ultrasound. The current method for activity detection is primarily visual 
inspection, and is subjective with a high degree of variability. Due to human variability, it is difficult to consistently apply the same decision criteria to different measurements. As an example, in Figure A.6 the vertical black dotted lines which indicate the potential onset of electrical activity. The first activity in the sEMG measurement occurs at EMG Start \#1, however it is followed by no other electrical activity. The EMG Start \#2 indicates another potential start of electrical activity as it is succeeded by sustained measured EMG activity. Further investigation is required to develop an unbiased and consistent methodology for electrical and mechanical activity detection.

The EMD reported from previous research varies considerably, with values from as low as $11 \mathrm{~ms}$ to as high as $125 \mathrm{~ms}[40,78]$. This is partly due to the diverse measurement methodology used by researchers [80] and difference in the muscle of interest. Different researchers use different methods of inducing the contraction using both voluntary and involuntary electric stimulation. Even within involuntary electric stimulation there are differences in the application of electrical stimulation levels [80]. There are also differences in definition of electrical or mechanical activity. The estimation of initial activity time is performed by a threshold approach. No set definition of threshold value has been agreed upon and thus different researchers use different thresholds in activity definition. As stated above, different measurement modalities are also used to measure the mechanical activity. The reported EMD values varied based on excitation modality, mechanical motion measurement, and threshold for activity detection. 


\section{References}

[1] J. Shi, Y. P. Zheng, X. Chen, and Q. H. Huang, "Assessment of muscle fatigue using sonomyography: Muscle thickness change detected from ultrasound images," Medical Engineering \& Physics, vol. 29, pp. 472-479, 2007

[2] J. C. Coggshall and G. A. Bekey, "EMG-Force dynamics in human skeletal muscle," Medical and Biological Engineering and Computing, vol. 8, no. 3, pp. 265-270, 2006

[3] M. A. Mañanas, R. Jané, J. A. Fiz, J. Morera, and P. Caminal, "Study of myographic signal from sternomastoid muscle in patients with chroni obstructive pulmonary disease," IEEE Transactions on Biomedical Engineering, vol. 47, no. 5, pp. 674-681, 2000

[4] P. K. Kasi, L. S. Krivickas, M. Meister, E. Chew, P. Bonata, M. Schmid, G. Karmen, L. $\mathrm{Pu}$, and E. A. Clancy, "Characterization of motor unit behavior in patients with amyotrophic lateral sclerosis," Proceedings of the 4th International IEEE EMBS Conference on Neural Engineering, pp. 10-13, 2009

[5] K. Akataki, K. Mita, and Y. Itoh, "Relationship between mechanomyogram and force during voluntary contraction reinvestigated using spectral decomposition," Eur. J. Appl. Physiol, vol. 80, pp. 173-179, 1999

[6] C. Orizio, "Surface mechanomyography," in Electromyography: Physiology, Engineering, and Noninvasive Application, R. Merletti and P. A. Parker, Eds., ed New Jersey: Wiley-IEEE Press, 2004, pp. 305-322.

[7] G. ÓLaighin, B. J. Broderick, M. Clarke-Moloney, F. Wallis, and P. A. Grace, "A technique for the computation of lower leg muscle volume from MRI images in the context of venous return," Proceedings of the 29th Annual International Conference of the IEEE EMBS, pp. 961-954, 2007

[8] T. Deffieux, J. Gennisson, M. Tanter, and M. Fink, "Ultrafast ultrasonic imaging of in vivo muscle contraction," IEEE Ultrasonics Symposium, pp. 1001-1004, 2006

[9] J.-Y. Guo, Y.-P. Zheng, Q.-H. Huang, and X. Chen, "Dynamic monitoring of forearm muscles using one-dimensional sonomyography system," Journal of Rehabilitation Research and Development, pp. 187-196, 2008

[10] K. Hoyt, B. Casteneda, and K. J. Parker, "Muscle Tissue Characterization using quantitative sonoelastography: preliminary results," IEEE Ultrasonics Symposium, pp. $365-368,2007$

[11] K. Hoyt, T. Kneezel, B. Casteneda, and K. J. Parker, "Quantitative Sonoelastography for the in vivo assessment of skeletal muscle viscoelasticity," Phys. Med. Biol, vol. 53, pp. 4063-4080, 2008

[12] N. R. Grubb, A. Fleming, G. R. Sutherland, and K. A. Fox, "Skeletal muscle contraction in healthy volunteers: assessment with Doppler tissue imaging," Radiology, vol. 194, no. 3, pp. 837-42, 1995

[13] J. Shi, J.-Y. Guo, Y.-J. Zhou, and Y.-P. Zheng, "Fatigue-induced continuous changes in muscle pennation angle during isometric contraction," 4th International Conference on Bioinformatics and Biomedical Engineering (iCBBE 210), pp. 1-4, 2010

[14] L. Herrington and S. Pearson, "The applicability of ultrasound imaging in the assessment of dynamic patella tracking: A preliminary investigation," The Knee, vol. 15, no. 2, pp. $125-127,2008$ 
[15] J. Shi, J.-Y. Guo, S.-X. Hu, and Y.-P. Zheng, "Recognition of Finger Flexion Motion from Ultrasound Image: A Feasibility Study," Ultrasound in medicine \& biology, vol. 38, no. 10 , pp. $1695-1704,2012$

[16] I. D. Loram, C. N. Maganaris, and M. Lakie, "Use of ultrasound to make noninvasive in vivo measurement of continuous changes in human muscle contractile length," $J \mathrm{Appl}$ Physiol, vol. 100, no. 4, pp. 1311-23, 2006

[17] J. G. A. Cashaback, T. Cluff, and J. R. Potvin, "Muscle fatigue and contraction intensity modulates the complexity of surface electromyography," Journal of Electromyography and Kinesiology, 2012

[18] Q. Zhou, Y. Chen, C. Ma, and X. Zheng, "Evaluation of upper limb muscle fatigue based on surface electromyography," SCIENCE CHINA Life Sciences, vol. 54, no. 10, pp. 939944,2011

[19] C. Lairamore, M. K. Garrison, W. Bandy, and R. Zabel, "Comparison of tibialis anterior muscle electromyography, ankle angle, and velocity when individuals post stroke walk with different orthoses," Prosthet Orthot Int, vol. 35, no. 4, pp. 402-10, 2011

[20] R. Brunner and J. Romkes, "Abnormal EMG muscle activity during gait in patients without neurological disorders," Gait \&amp; Posture, vol. 27, no. 3, pp. 399-407, 2008

[21] E. Al-Zahrani, C. Gunasekaran, M. Callaghan, P. Gaydecki, D. Benitez, and J. Oldham, "Within-day and between-days reliability of quadriceps isometric muscle fatigue using mechanomyography on healthy subjects," Journal of Electromyography and Kinesiology, vol. 19, no. 4, pp. 695-703, 2009

[22] S. Kawakami, N. Kodama, N. Maeda, S. Sakamoto, K. Oki, Y. Yanagi, J.-I. Asaumi, T. Maeda, and S. Minagi, "Mechanomyographic activity in the human lateral pterygoid muscle during mandibular movement," Journal of Neuroscience Methods, vol. 203, no. 1, pp. 157-162, 2012

[23] Y. Ohta, N. Shima, and K. Yabe, "In vivo behaviour of human muscle architecture and mechanomyographic response using the interpolated twitch technique," Journal of Electromyography and Kinesiology, vol. 19, no. 3, pp. e154-e 161, 2009

[24] J. Silva, W. Heim, and T. Chau, "MMG-based classification of muscle activity for prosthesis control," Engineering in Medicine and Biology Society, 2004. IEMBS '04. 26th Annual International Conference of the IEEE, vol. 1, pp. 968-971, 2004

[25] T. W. Beck, T. Housh, A. C. Fry, J. T. Cramer, J. Weir, B. Schilling, M. Falvo, and C. Moore, "MMG-EMG Cross Spectrum and Muscle Fiber Type," Int J Sports Med, vol. 30, no. 07, pp. 538-544, 2009

[26] L. Qi, J. M. Wakeling, and M. Ferguson-Pell, "Spectral properties of electromyographic and mechanomyographic signals during dynamic concentric and eccentric contractions of the human biceps brachii muscle," Journal of Electromyography and Kinesiology, vol. 21 , no. 6, pp. 1056-1063, 2011

[27] S. R. Perry-Rana, T. J. Housh, G. O. Johnson, A. J. Bull, J. M. Berning, and J. T. Cramer, "MMG and EMG responses during fatiguing isokinetic muscle contractions at different velocities," Muscle \& Nerve, vol. 26, no. 3, pp. 367-373, 2002

[28] J.-Y. Guo, Y.-P. Zheng, H.-B. Xie, and X. Chen, "Continuous monitoring of electromyography (EMG), mechanomyography (MMG), sonomyography (SMG) and torque output during ramp and step isometric contractions," Medical Engineering \&amp; Physics, vol. 32, no. 9, pp. 1032-1042, 2010 
[29] E. L. Finanger, B. Russman, S. C. Forbes, W. D. Rooney, G. A. Walter, and K. Vandenborne, "Use of Skeletal Muscle MRI in Diagnosis and Monitoring Disease Progression in Duchenne Muscular Dystrophy," Physical Medicine and Rehabilitation Clinics of North America, vol. 23, no. 1, pp. 1-10, 2012

[30] A. Magot, F. Caillon, J. M. Mussinia, A. David, and Y. Pereon, "D.P.17 Whole body muscle MRI in three patients suffering from XMEA: A peculiar involvement pattern," Neuromuscular Disorders, vol. 22, no. 9-10, p. 823, 2012

[31] M. Froeling, A. J. Nederveen, D. F. R. Heijtel, A. Lataster, C. Bos, K. Nicolay, M. Maas, M. R. Drost, and G. J. Strijkers, "Diffusion-tensor MRI reveals the complex muscle architecture of the human forearm," Journal of Magnetic Resonance Imaging, vol. 36, no. 1, pp. 237-248, 2012

[32] P. Huijing, A. Yaman, C. Ozturk, and C. Yucesoy, "Effects of knee joint angle on global and local strains within human triceps surae muscle: MRI analysis indicating in vivo myofascial force transmission between synergistic muscles," Surgical and Radiologic Anatomy, vol. 33, no. 10, pp. 869-879, 2011

[33] A. C. Gellhorn, D. C. Morgenroth, and B. Goldstein, "A novel sonographic method of measuring patellar tendon length," Ultrasound in Med. \& Biol, vol. 38, no. 5, pp. 719726,2012

[34] M. Rana and J. M. Wakeling, "In-vivo determination of 3D muscle architecture of human muscle using free hand ultrasound," Journal ofBiomechanics, vol. 44, pp. 2129-2135, 2011

[35] C. Kasai, K. Namekawa, A. Koyano, and R. Omoto, "Real time two-dimensional blood flow imaging using an autocorrelation technique," IEEE Transactions on Sonics and Ultrasonics, vol. SU-32, no. 3, pp. 458-464, 1985

[36] T. Loupas, J. T. Powers, and R. W. Gill, "An axial velocity estimator for ultrasound blood flow imaging, based on a full evaluation of the Doppler equation by means of a two-dimensional autocorrelation approach," IEEE Transaction on Ultrasonics, Ferroelectrics and Frequency Control, vol. 42, no. 4, pp. 672-688, 1995

[37] X. Lai, H. Torp, and K. Kristoffersen, "An extended autocorrelation method for estimation of blood velocity," IEEE Transaction on Ultrasonics, Ferroelectrics and Frequency Control, vol. 44, no. 6, pp. 1332-1342, 1997

[38] H. Zhao, P. Song, M. W. Urban, J. F. Greenleaf, and S. Chen, "Shear wave speed measurement using an unfocused ultrasound beam," Ultrasound in Med. \& Biol, vol. 38, no. 9 , pp. 1646-1655, 2012

[39] T. Miwa, Y. Yoshihara, K. Kanzawa, R. K. Parajuli, and Y. Yamakoshi, "Tissue viscoelasticity measurement system by simultaneous multiple-frequency excitation," Japanese Journal of Applied Physics, vol. 51, pp. 07GF13-1 - 07GF13-5, 2012

[40] A. Nordez, T. Gallot, S. Catheline, A. Guével, C. Cornu, and F. Hug, "Electromechanical delay revisited using very high frame rate ultrasound," Journal of Applied Physiology, vol. 106, no. 6, pp. 1970-1975, 2009

[41] E. J. Chen, W. K. Jenkins, and J. W. D. O'Brien, "The accuracy and precision of estimating tissue displacements from ultrasonic images," Ultrasonics Symposium, 1992. Proceedings., IEEE 1992, pp. 1069-1072 vol.2, 1992

[42] E. J. Chen, W. K. Jenkins, and J. W. D. O'Brien, "The impact of various imaging parameters on ultrasonic displacement and velocity estimates," Ultrasonics. 
Ferroelectrics and Frequency Control, IEEE Transactions on, vol. 41, no. 3, pp. 293301,1994

[43] R. G. P. Lopata, M. M. Nillesen, H. H. G. Hansen, I. H. Gerrits, J. M. Thijssen, and C. L. d. Korte, "Performance Evaluation of Methods for Two-Dimensional Displacement and Strain Estimation Using Ultrasound Radio Frequency Data," Ultrasound in medicine \& biology, vol. 35, no. 5, pp. 796-812, 2009

[44] F. W. Kremkau, Diagnostic Ultrasound: Principles and Instruments, 7 ed. Missouri, U.S.A.: Saunders Elesvier, 2006.

[45] P. Hoskins, A. Thrush, K. Martin, and T. Whittingam, Diagnostic Ultrasound: Physics and equipment. London, England: Greenwich Medical Media Limited, 2003.

[46] W. R. Hedrick, D. L. Hykes, and D. E. Starchman, Ultrasound Physics and Instrumentation, 4 ed. Missouri, U.S.A: Elesvier Mosby, 2005.

[47] J. L. Prince and J. M. Links, Medical Imaging: Signals and Systems. Upper Saddle River, NJ: Pearson Prentice Hall, 2006.

[48] N. Feng, J. Zhang, and W. Wang, "A quadrature demodulation method based on tracking the ultrasound echo frequency," vol. 44, pp. 47-50, 2006

[49] M. E. Anderson, "A heterodyning demodulation technique for spatial quadrature," IEEE Ultrasonics Symposium, pp. 1487-1451, 2000

[50] M. E. Anderson, "Multi-dimensional velocity estimation with ultrasound using spatial quadrature," IEEE Transaction on Ultrasonics, Ferroelectrics and Frequency Control, vol. 45 , no. 3 , pp. $852-861,1998$

[51] P. Levesque and M. Sawam, "New digital quadrature demodulator for real-time handheld ultrasound medical imaging device," IEEE International Symposium on Circuits and Systems, pp. 2949-2952, 2008

[52] G. F. Pinton, J. J. Dahl, and G. E. Trahey, "Rapid tracking of small displacements with ultrasound," IEEE Transaction on Ultrasonics, Ferroelectrics and Frequency Control, vol. 53, no. 6, pp. 1103-1117, 2006

[53] S. H. Chang, S. B. Park, and G. H. Cho, "Phase-error-free quadrature sampling technique in the ultrasonic B-Scan imaging system and its application to the synthetic focusing system," IEEE International Symposium on Circuits and Systems, vol. 40, no. 3, pp. 216223,1993

[54] H. Trop, K. Kristoffersen, and B. A. J. Andelsen, "Autocorrelation techniques in color imaging: signal model and statistical properties of the autocorrelation estimates," IEEE Transaction on Ultrasonics, Ferroelectrics and Frequency Control, vol. 41, no. 5, pp. 604-612, 1994

[55] H. Hasegawa and H. Kanai, "Reduction of influence of variation in center frequencies of RF echoes on estimation of artery-wall strain," IEEE Transaction on Ultrasonics, Ferroelectrics and Frequency Control, vol. 55, no. 9, pp. 1921-1934, 2008

[56] V. Shamdasani and Y. Kim, "Two-dimensional autocorrelation method for ultrasoundbased strain estimation," Proceedings of the 26th Annual International Conference of the IEEE EMBS, vol. 1, pp. 1380-1383, 2004

[57] S. I. Rabben, S. Bjærum, V. Sørhus, and H. Torp, "Ultrasound-based vessel wall tracking: an autocorrelation technique with RF center frequency estimation," Ultrasound in Med. \& Biol, vol. 28, no. 4, pp. 507-517, 2002 
[58] J. Silver, Y. Ono, and A. Adler, "An ultrasonic technique for imaging of tissue motion due to muscle contraction," Ultrasonics Symposium (IUS), 2009 IEEE International, pp. 2441-2444, 2009

[59] R. L. Maurice and M. Bertrand, "Speckle-Motion Artifact Under Tissue Shearing," IEEE Transaction on Ultrasonics, Ferroelectrics and Frequency Control, vol. 46, no. 3, pp. 584-594, 1999

[60] F. Kallel, M. Bertrand, and J. Meunier, "Speckle Motion Artifact Under Tissue Rotation," IEEE Transactions on Ultrasonics, Ferroelectrics, and Frequency Control, vol. 41, no. 1, pp. 105-122, 1994

[61] Esaote $\mathcal{C}$ Europe, "Picus User Manual: Instructions for Use and Technical Description", 2008.

[62] G. S. Kino, Acoustic waves: devices, imaging, and analog signal processing: PrenticeHall, 1987.

[63] R. Demirli and J. Saniie, "Model-based estimation of ultrasonic echoes. Part I: Analysis and algorithms," Ultrasonics, Ferroelectrics and Frequency Control, IEEE Transactions on, vol. 48, no. 3, pp. 787-802, 2001

[64] Q. Zhang, G. Yang, and P. Que, "Ultrasonic signals processing base on parameters estimation," Russian Journal of Nondestructive Testing, vol. 45, no. 1, pp. 61-66, 2009

[65] F. Vakhshiteh, "Parameter Estimation Techniques for Ultrasound Phase Reconstruction," M.A.Sc. thesis, Dept. Sys. and Com. Eng., Carleton Univ., Ottawa, ON, 2010.

[66] S. C. Gandevia, G. M. Allen, and D. K. McKenzie, "Central fatigue. Critical issues, quantification and practical implications," Adv Exp Med Biol, vol. 384, pp. 281-294, 1995

[67] U. Granacher, I. Wolf, A. Wehrle, S. Bridenbaugh, and R. Kressig, "Effects of muscle fatigue on gait characteristics under single and dual-task conditions in young and older adults," Journal of NeuroEngineering and Rehabilitation, vol. 7, no. 1, p. 56, 2010

[68] B. Elfving, $\AA$. Dedering, and G. Németh, "Lumbar muscle fatigue and recovery in patients with long-term low-back trouble-electromyography and health-related factors," Clinical Biomechanics, vol. 18, no. 7, pp. 619-630, 2003

[69] V. Balasubramanian, A. Dutt, and S. Rai, "Analysis of muscle fatigue in helicopter pilots," Applied Ergonomics, vol. 42, no. 6, pp. 913-918, 2011

[70] M. J. Zwarts and D. F. Stegeman, "Multichannel surface EMG: Basic aspects and clinical utility," Muscle \& Nerve, vol. 28, no. 1, pp. 1-17, 2003

[71] L. Mademli and A. Arampatzis, "Behaviour of the human gastrocnemius muscle architecture during submaximal isometric fatigue," European Journal of Applied Physiology, vol. 94, no. 5, pp. 611-617, 2005

[72] E. Henneman, G. Somjen, and D. O. Carpenter, "Functional significance of cell size in spinal motoneurons," Journal of Neurophysiology, vol. 28, no. 3, pp. 560-580, 1965

[73] S. J. Garland, R. M. Enoka, L. P. Serrano, and G. A. Robinson, "Behavior of motor units in human biceps brachii during a submaximal fatiguing contraction," Journal of Applied Physiology, vol. 76, no. 6, pp. 2411-2419, 1994

[74] A. F. Huxley, "Muscle structure and theories of contraction," Prog. Biophys. Mol. Biol., vol. 7, pp. 255-318, 1957

[75] M. Narici, "Human skeletal muscle architecture studied in vivo by non-invasive imaging techniques: functional significance and applications," Journal of electromyography and kinesiology : official journal of the International Society of Electrophysiological Kinesiology, vol. 9, no. 2, pp. 97-103, 1999 
[76] A. Georgoulis, S. Ristanis, A. Papadonikolakis, E. Tsepis, U. Moebius, C. Moraiti, and N. Stergiou, "Electromechanical delay of the knee extensor muscles is not altered after harvesting the patellar tendon as a graft for ACL reconstruction: implications for sports performance," Knee Surgery, Sports Traumatology, Arthroscopy, vol. 13, no. 6, pp. 437443,2005

[77] P. R. Cavanagh and P. V. Komi, "Electromechanical delay in human skeletal muscle under concentric and eccentric contractions," Eur. J. Appl. Physiol. Occup. Physiol, vol. 42 , pp. $159-163,1979$

[78] J. T. Blackburn, D. R. Bell, M. F. Norcross, J. D. Hudson, and L. A. Engstrom, "Comparison of hamstring neuromechanical properties between healthy males and females and the influence of musculotendinous stiffness," Journal of electromyography and kinesiology : official journal of the International Society of Electrophysiological Kinesiology, vol. 19, no. 5, pp. e362-e369, 2009

[79] E. Winter and F. Brookes, "Electromechanical response times and muscle elasticity in men and women," European Journal of Applied Physiology and Occupational Physiology, vol. 63, no. 2, pp. 124-128, 1991

[80] S. U. Yavuz, A. Şendemir-Ürkmez, and K. S. Türker, "Effect of gender, age, fatigue and contraction level on electromechanical delay," Clinical neurophysiology : official journal of the International Federation of Clinical Neurophysiology, vol. 121, no. 10, pp. 17001706,2010

[81] H.-Y. Chen, J.-J. Liau, C.-L. Wang, H.-J. Lai, and M.-H. Jan, "A Novel Method for Measuring Electromechanical Delay of the Vastus Medialis Obliquus and Vastus Lateralis," Ultrasound in Medicine \&amp; Biology, vol. 35, no. 1, pp. 14-20, 2009

[82] B. D. Moore, J. Drouin, B. M. Gansneder, and S. J. Shultz, "The differential effects of fatigue on reflex response timing and amplitude in males and females," Journal of Electromyography and Kinesiology, vol. 12, no. 5, pp. 351-360, 2002 